\title{
Development and application of LC-MS-based differential
}

\section{metabolic profiling in plant systems}

\author{
Dissertation \\ zur Erlangung des Doktorgrades \\ der Fakultät für Agrarwissenschaften \\ der Georg-August-Universität Göttingen
}

vorgelegt von

Astrid Ratzinger

geboren in Berlin

Göttingen, im Mai 2008 
D 7

1. Referent: Prof. Dr. Petr Karlovsky

2. Korreferent: Prof. Dr. Andreas von Tiedemann

Tag der mündlichen Prüfung: 17. Juli 2008 


\section{Contents}

Chapter 1: General introduction (Literature review) ....................................... 1

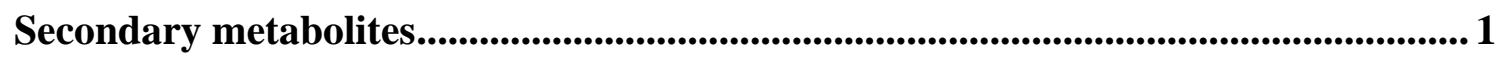

Plant metabolomics ..................................................................................................................... 3

Introduction into terms and definitions used in metabolomics ................................. 3

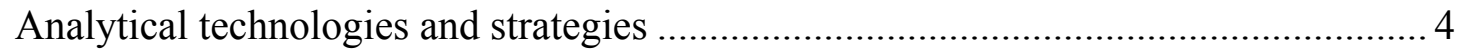

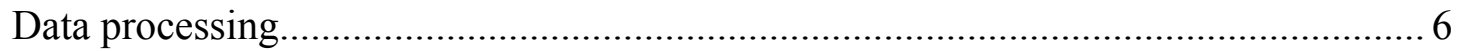

Application of metabolomics in biology and agricultural research........................... 8

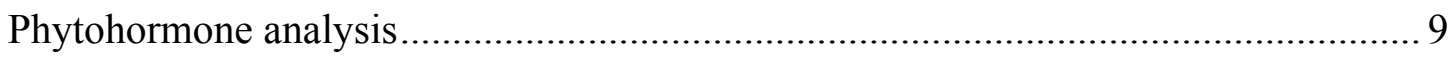

The pathosystem Verticillium longisporum/Brassica spp. ............................................. 11

Chemical signals in $V$. longisporum/B. napus interaction .......................................... 12

Putative roles of secondary metabolites of $V$. longisporum ................................... 12

Plant defense reactions after Verticillium infection............................................... 13

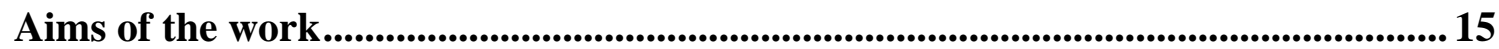

\section{Chapter 2: Differential metabolic profiling of xylem sap of Brassica} napus infected with Verticillium longisporum ...................................................16

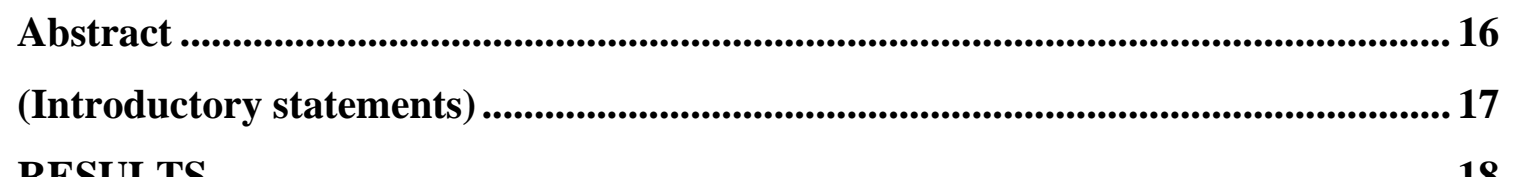

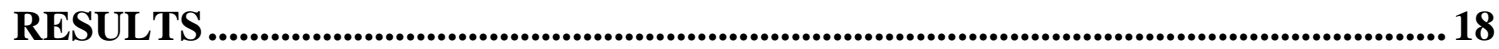

Nontargeted differential metabolic profiling of pooled xylem sap samples ............... 18

Analysis of xylem sap from individual plants infected with $V$. longisporum and

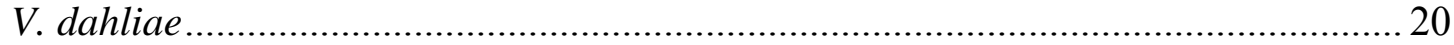

Changes in metabolite levels and fungal DNA content in the course of infection...... 21

Correlations between intensities of infection-specific signals .................................. 22

Characterization of infection-specific metabolic signals.......................................... 22

DISCUSSION.................................................................................................................... 23

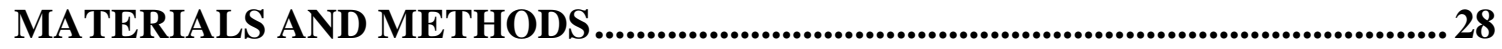

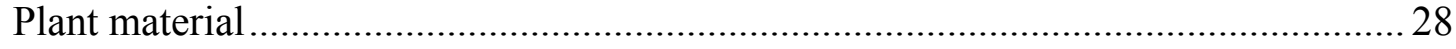

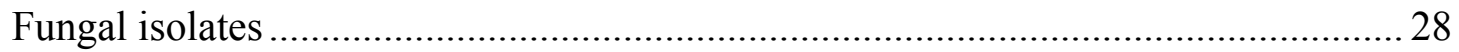

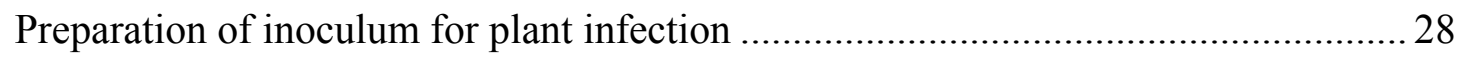

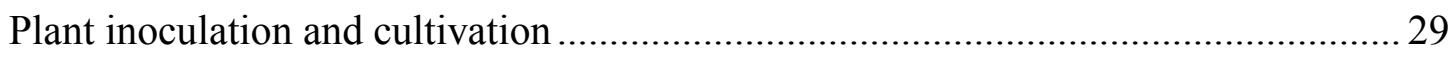




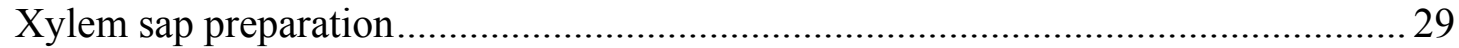

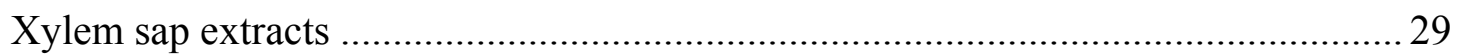

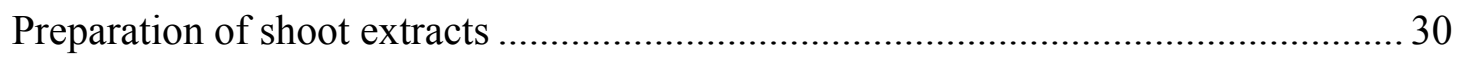

Preparation of culture supernatants from $V$. longisporum ...................................... 30

High performance liquid chromatography (HPLC) and mass spectrometric (MS)

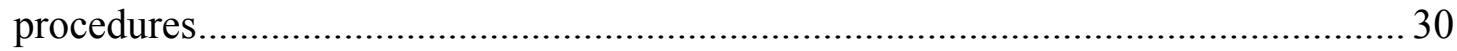

Selective ion monitoring and product ion scan using triple quadrupole MS.............. 31

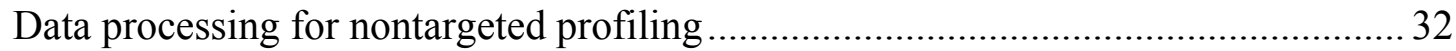

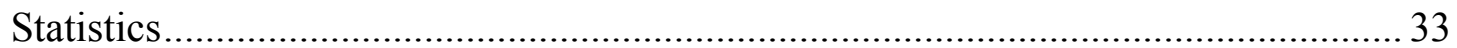

Quantification of $V$. longisporum DNA in hypocotyls and shoots of B. napus .......... 33

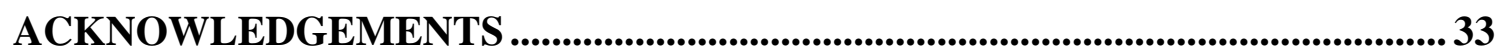

LITERATURE CITED ................................................................................................. 34

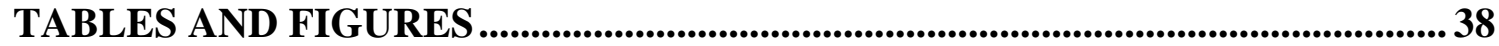

\section{Chapter 3: Identification of salicylic acid glucoside in xylem sap of Brassica napus and the effect of infection with Verticillium longisporum}

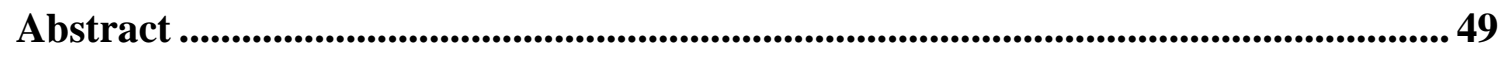

Introduction ........................................................................................................................... 50

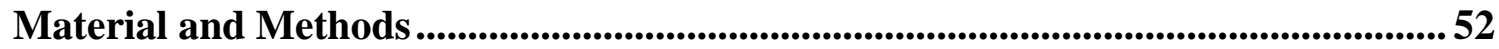

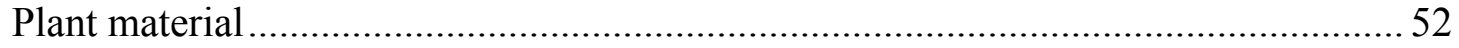

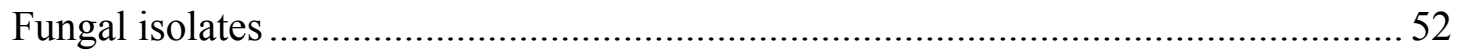

Preparation of inoculum for plant infection ........................................................... 52

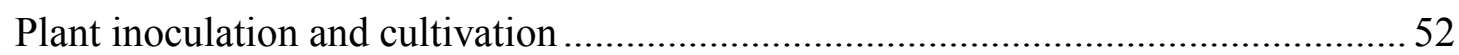

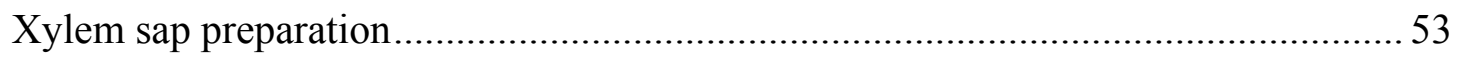

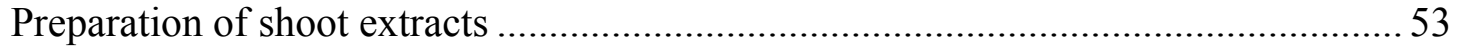

High performance liquid chromatography and mass spectrometry …........................53

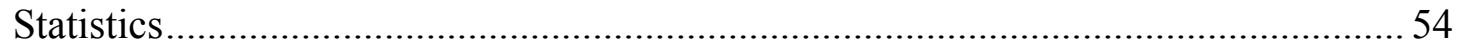

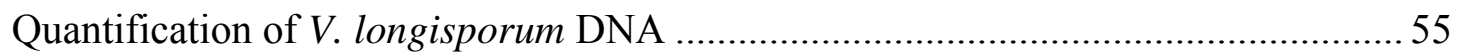

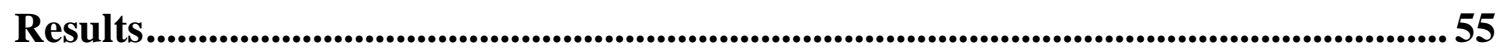

Salicylic acid glucoside (SAG) in xylem sap and shoots of Brassica napus .............. 55

Relationship between salicylic acid and its glucoside in xylem sap and shoots and the

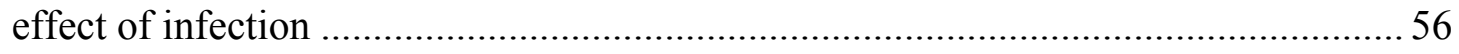


Changes in the concentration of SA, SAG, JA, and ABA in xylem sap at different times after infection

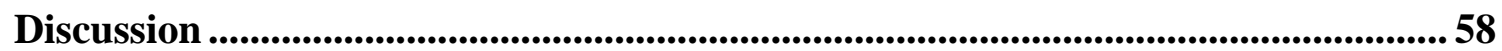

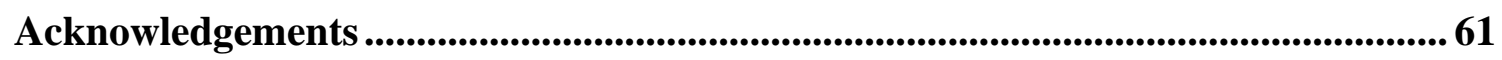

References ............................................................................................................................ 61

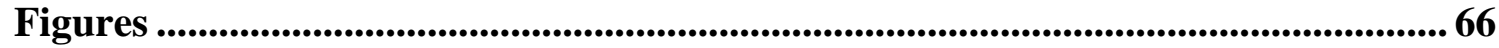

Chapter 4: Relationship between metabolic and genomic diversity in sesame (Sesamum indicum L.)

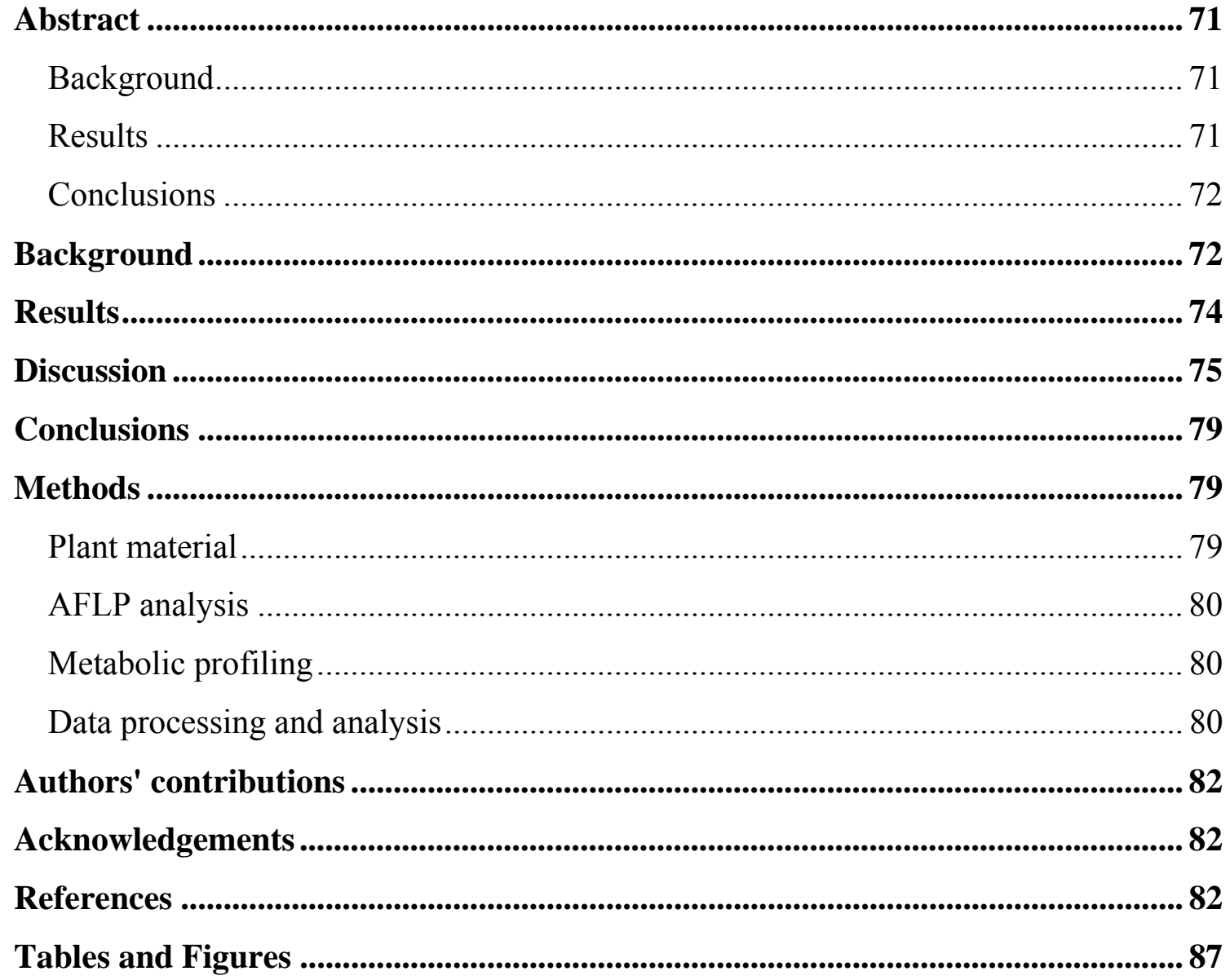

Chapter 5: Identification of soil $P$ mobilizing components of sugar beet root exudates after differential metabolic profiling by HPLC-MS ..........93

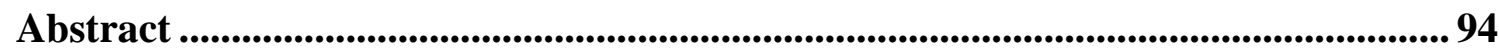

Introduction ................................................................................................................................. 95

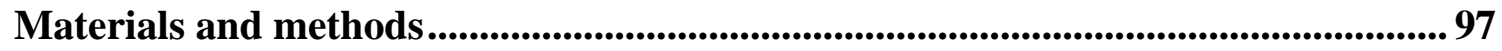

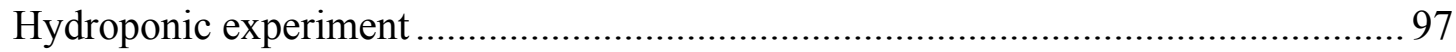




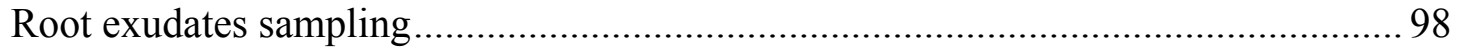

Preparation of samples and standards for HPLC-MS analysis .................................. 98

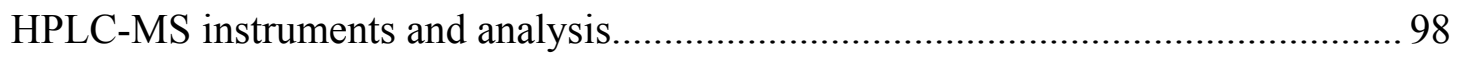

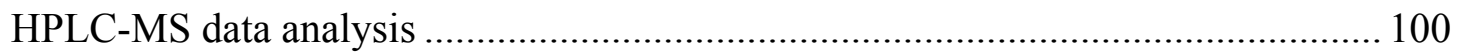

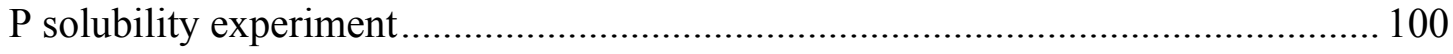

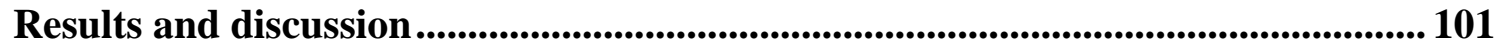

Shoot dry weight and shoot P concentration ........................................................ 101

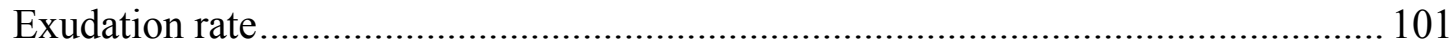

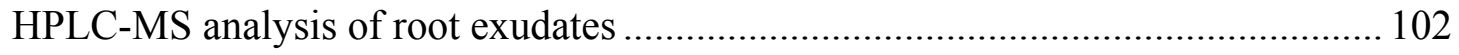

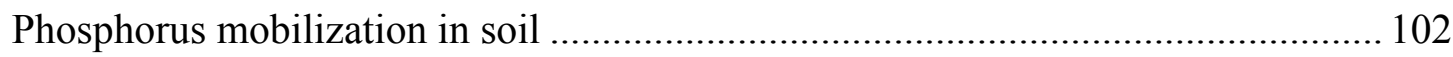

Identification of salicylic and citramalic acids in root exudates ............................. 103

References .......................................................................................................................... 104

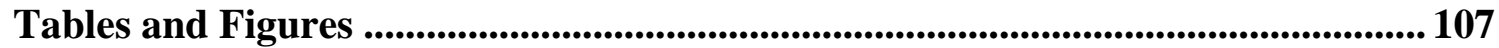

\section{Chapter 6: General Discussion ............................................................................. 112}

Evaluation of analytical strategies and data processing tools in application to different plant systems ................................................................................................................. 112

Nontargeted metabolic profiling approaches and resulting follow-up analytics....... 112

Applicability of CODA algorithm and XCMS software for data processing............ 114

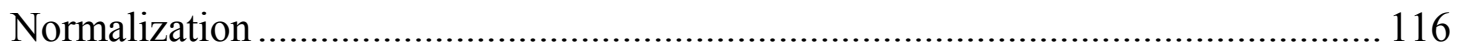

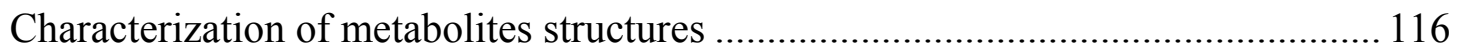

Putative structures of signals in xylem sap of $B$. napus after $V$. longisporum

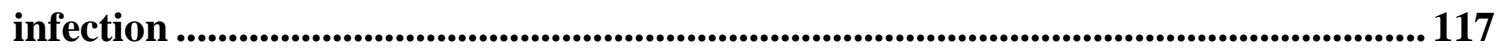

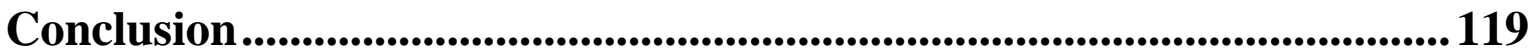

Summary …............................................................................................................. 120

References (cited in general introduction and general discussion) ......... 122

Publications from this work ................................................................................. 139

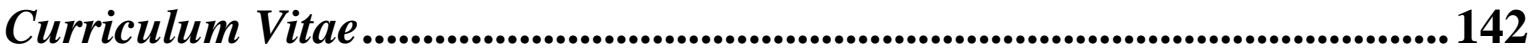




\section{Chapter 1: General introduction (Literature review)}

\section{Secondary metabolites}

Plants and microorganisms produce a vast number of natural compounds known as secondary metabolites. To distinguish these compounds from primary metabolites, Kossel (1891) introduced the concept of "secondary metabolites", these products are not necessary for the growth, survival or reproduction of their producers but benefit the organism in other ways. Secondary metabolites display an enormous structural diversity; however each of them is synthesized only by a limited taxonomic group of organisms. Primary metabolites, in contrast, are found in all living organisms since they perform essential functions in growth and development.

Most secondary metabolites have highly diverse biological functions that are still unexplored, since this type of "insignificant" metabolism was of no interest to biologist and natural product chemistry was limited for a long time to structure elucidation and drug development. The situation has changed over the last three decades: Many secondary metabolites now have been demonstrated to play an ecological role, being involved in (pathogenic) interactions between plants and microorganisms or in communication with a symbiont (Kutchan 2001). In the interaction of a plant with its abiotic and biotic environment, secondary metabolites provide protection against abiotic stress (such as ozone, UV light, cold, drought, heavy metals, or nutrient deficiency) or selective defense against herbivory and pathogen infection (Walling 2000; Dixon 2001).

One of the best studied classes of low-molecular-weight plant secondary metabolites represent phytoalexines, which are produced by plants de novo in response to diverse forms of stress, and pre-formed constitutive defense chemicals called phytoanticipins. Both phytoalexins and phytoanticipins are part of the plants' defense mechanism as they inhibit the spreading of fungal mycelia or bacterial cells within the plant (Hammerschmidt 1999; Grayer and Kokubun 2001).

Apart from their function in responses to environmental stress, plant secondary metabolites are synthesized to promote symbioses with beneficial microbes, insects and higher animals (Demain and Fang 2000). Furthermore, certain secondary metabolites produced by plants 
as well as microbes manipulate the solubilization, transport, and up-take of metal ions to overcome nutrient deficiency (Guerinot 1994; Demain and Fang 2000; Staiger 2002). Secondary metabolites also play a crucial role as internal signals involved in plant development and growth. For instance, plant hormones regulate essential physiological and developmental processes in the plant but can also function when transmitted between plants or other species (Shulaev et al. 1997). It has been demonstrated for many plant species that in mycorrhizal symbiosis the release of phytohormones (indole acetic acid, cytokinins) by the plants or microbes can promote the growth of their partner by enhancing their nutrient acquisition (Costacurta and Vanderleyden 1995; Hause et al. 2007).

Plant pathogens often synthesize secondary metabolites with phytotoxic or phytohormonlike effects which act as virulence factors by manipulating the host plant metabolism or with suppressive effects which disable or neutralise the defense reaction of the plant. The beneficial effect for the phytotoxin producing microorganism is an increased population of plant tissue with a longer duration (Demain and Fang 2000). Many of the fungal secondary metabolites are highly toxic to animals (mycotoxins) and/or exhibit antibiotic activity against other microorganisms. Although there are strong indications that plant pathogenic fungi have developed certain mycotoxins to increase their virulence on host plant tissue, the specific function of most mycotoxins in the survival of fungi is yet unknown (Desjardins et al. 1993). Some mycotoxins act as pathogenicity factors or virulence factors (Hof 2008).

Apart from undesirable effects of virulence/pathogenicity factors and mycotoxins, many secondary metabolites are important health-promoting components of food and feedstuff as provitamins, natural preservatives, antioxidants, agents depressing cholesterol levels and stimulants of the immune system (Demain and Fang 2000). Secondary metabolites are largely responsible for the taste, aroma and colour of food products and one of the major quality factors (Memelink 2005; Hall 2006). Without secondary metabolites, spices and many other plant products such as sesame seeds and fruit bodies of truffles (Splivallo et al. 2007) would become useless. Finally, the immense structural variety of secondary metabolites serves as a source of lead structures for active compounds in the pharmaceutical industry and plant protection.

Understanding biological functions of secondary metabolites is a fundamental prerequisite for progress in the ecology of plant pathogens, symbionts and saprophytes, but it also 
facilitates the development of practical applications in resistance breeding, plant protection and biotechnology.

In this work, four groups of secondary metabolites of plant origin or metabolites involved in biotic plant interactions were investigated: (i) xylem metabolites involved in interaction between plant and a pathogenic fungus, (ii) phytohormones produced by plants during pathogenicity, (iii) sesame metabolites involved in quality and resistance traits, and (iv) root exudates facilitating the uptake of phosphorus. The development and application of nontargeted plant metabolomic techniques appeared to be promising in these contexts.

\section{Plant metabolomics}

\section{Introduction into terms and definitions used in metabolomics}

In the last 10 years, when the term 'metabolome' was created to describe the metabolite complement of a living organism in a particular physiological or developmental state (Oliver et al. 1998), metabolomics has evolved to a central tool in plant physiology, biochemistry and ecology.

Metabolomics is the third major concept in functional genomics besides transcriptomics (mRNA profiling) and proteomics (Fiehn 2002). Metabolomic analyses are classified into four different approaches (Fiehn 2002; Hall 2006): i) Metabolite target analysis describes the classical target-driven survey or quantification of one or a few single compounds by using optimized extraction and dedicated separation and detection techniques. ii) Metabolic fingerprinting aims at generating static profiles of anonymous signals, which are characteristic for a given sample and can be used for genotype discrimination or confirmation. iii) Metabolic profiling generates profiles of signals, including either anonymous or identified metabolites, and is used to understand biological processes responsible for differences in the content of metabolites among comparative samples, such as healthy/diseased organisms, treated/control cultures, mutant/wildtype genotypes, or different cultivars. Identification and quantification is limited to a number of analytes chosen on the basis of discriminant analysis, or on molecular relationships. The profiling of metabolite classes is used to describe quantitatively the representation of selected substances belonging to a set of chemically related compounds, or a certain biochemical pathway (e.g., phytohormones, oxylipins, or glucosinolates) in the sample. iv) Metabolomics designates the comprehensive unbiased detection, identification, and 
quantification of all metabolites in a biological system. This is no easy challenge considering the immense metabolite diversity observed in the plant kingdom, up to 200,000 different metabolites (Fiehn 2002) and additionally the metabolome is highly dependent on the environment and the developmental stage of the organism. Despite this, improved analytical technologies combined with newly developed, dedicated data mining, statistical, and bioinformatic strategies are the key to accomplish this and make metabolomics as an indispensable tool in plant biology.

\section{Analytical technologies and strategies}

The rapid development and improvement of analytical technologies, particularly mass spectrometry (MS), has enabled the detection and identification of a large number of metabolites simultaneously in a sensitive and high-throughput manner.

Gas- and liquid based chromatography in combination with various types of MS detection and nuclear magnetic resonance spectroscopy (NMR) has been successfully applied to metabolomics. Gas chromatography (GC) coupled with mass spectrometers played a major role in the pioneering profiling studies and high-throughput metabolite analyses in the past (Roessner et al. 2002). GC provides a highly efficient and robust separation of compound mixtures. In combination with MS, separated metabolites can be detected in a very accurate, selective and sensitive way allowing their identification and quantification by their specific mass spectrum. Therefore GC-MS has been employed for metabolite analysis in many different plant species, such as Arabidopsis thaliana (Fiehn et al. 2000), Medicago truncatula (Duran et al. 2003), and Solanum tuberosum (Roessner et al. 2000). However, every analytical technology has its limitations as to the type of compounds which can be separated and detected. GC is predominantly used for unpolar low molecular weight compounds which are volatile or have to be converted into volatile derivatives prior analysis. For more comprehensive profiling of metabolites complementary techniques such as liquid chromatography (LC) are required (Roessner et al. 2000; Kopka et al. 2004). The application of LC coupled to electrospray ionization (ESI) MS enables the separation and quantification of compounds with medium polarity, but also highly polar, thermo-labile and high-molecular weight molecules. The advantage of MS detection over traditional ultraviolet or visible light (UV/VIS) absorbance, light scattering or refraction index detectors is the reasonably high selectivity for most metabolites, the unbiased detection, 
and the structural information provided by the mass spectrum. The combination of LC and MS comprises soft ionization (by electrospray) and high mass accuracy to generate information about molecular ions or characteristic fragments of the unknown compounds (Niessen 1999). This multidimensional technique has been applied successfully in plant metabolomic studies, e.g. by using ion trap MS (Huhman and Sumner 2002). By use of capillary LC coupled to ESI quadrupole time-of-flight MS, 1,400 mass signals (based on $\mathrm{m} / \mathrm{z}$ value) could be detected in Arabidopsis thaliana extracts (Roepenack-Lahaye et al. 2004). The application of direct injection ESI-Fourier Transform-Ion Cyclotron Resonance (FT-ICR)/MS yielded in up to 5,000 signals in a single plant extract demonstrating the suitability for high-throughput metabolic fingerprinting of large sets of biological samples for metabolic changes (Aharoni et al. 2002). Recently, a metabolic phenotyping scheme on the basis of FT-ICR/MS was developed and revealed metabolic alterations in A. thaliana seedlings treated with different herbicides (Oikawa et al. 2006; Ohta et al. 2007). Very recently, it has been suggested that using multiple ionization technologies (ESI/Atmospheric Pressure Chemical Ionization/ matrix-assisted laser desorption/ionization [MALDI]) combined with MS can cover the inherent metabolite diversity and therefore the complexity in metabolomic studies (Nordström et al. 2008).

An additional important requirement for metabolite analysis is sensitivity suitable for the detection of low-concentrated metabolites from very small samples, which enables the investigation of tissue-specific distribution of metabolites at cell and organ level. For example, for spatial profiling of phytochemicals in different tissue regions of the stem, such as xylem and phloem, MALDI-MS was employed which can desorb and ionize analytes from solid samples even at up to femtomole levels ( $\mathrm{Ng}$ et al. 2007).

The identification of the signals detected in LC-MS is difficult because of the lack of spectral databases and the commercial availability of plant compounds as reference standards. LC-MS generated mass spectra are typically instrument dependent thus the use of spectral LC-MS libraries is limited. With the information about the mass of a metabolic signal, NIST or KEGG database (Goto et al. 2002; Kanehisa et al. 2002) can be refered to suggestions as to the structure, but numerous entries are related to medicine. Multidimensional techniques (MS/MS or MS/NMR) quickly provide structural information leading to preliminary identification of the compound. For instance, stepwise fragmentation $\left(\mathrm{MS}^{\mathrm{n}}\right)$ leads to information about fragment ions which contributes to structural elucidation. Nevertheless, the final identity of the compound can only be 
revealed by analysis of authentic standard compounds or analysis of fractionated and purified sample by NMR. The most powerful hyphenation which provides on-line structural investigation of plant metabolites represents LC-MS-NMR (Wolfender et al. 2003), but is limited by the low sensitivity of the NMR instruments used in this combination.

Other metabolic profiling tasks rely on sets of chemically anonymous signals and achieve their goals without structure elucidation (see next paragraph). Relative quantification of ratios of signal intensities between comparative experiments is used in metabolic profiling, since the quantification of the absolute amounts of all detected metabolites is difficult to achieve with the help of external or internal calibration standards for a number of highly diverse or even unknown compounds. Another approach for relative quantification of unknown components in comparative samples is in vivo labelling of reference samples using stable isotopes (Birkemeyer et al. 2005).

\section{Data processing}

The study of metabolites based on hyphenated MS techniques creates large amounts of three-dimensional (retention time - mass-to-charge ratio $[\mathrm{m} / \mathrm{z}$ value $]$ - intensity) data for a large number of samples. The ability to sift through this data, to extract the most meaningful parts of the data and to perform reliable, comparative analyses are the key to conducive new knowledge in plant metabolomics (Goodacre 2005). Raw data processing is the most challenging and time consuming step in metabolomic data analysis (Shulaev 2006).

Manual data analysis of each single chromatogram is time consuming and tedious work. For rapid and unbiased analysis of large data sets from samples, automated data processing is required including raw data conversion into a universal data format, noise reduction, peak detection and integration, retention time alignment, normalization of intensities, and comparative analysis and visualization strategies (Shulaev 2006). Conversion into a universal data format is necessary since each mass spectrometer software has an own file format. The alignment of retention time corrects for differences in chromatographic behaviour caused by column aging, variation of the quality of the mobile phase and temperature variation. Normalization of the signal intensity will compensate differences in the efficiency of ionization and detection and sample amount. Comparative analysis 
includes statistical tools to identify subtle differences between samples of the data sets. A comprehensive unsupervised automated solution for data processing does not exist yet. The software supplied with MS detectors is usually unsuitable for these tasks; therefore the usage of commercial and/or free available open source software packages is essential. Peak alignment procedures play a crucial role in metabolomic studies. In the last few years, many programs for unbiased peak extraction and alignment for GC-MS and LC-MS data have emerged, providing solutions which automatically import, align, correct the baseline and export large chromatographic data sets in numerical form and subsequent statistics. To date, approximately 30 alignment programs have been published (according to http://fiehnlab.ucdavis.edu/staff/kind/Metabolomics/Peak_Alignment/). Among the most importants are MetAlign (Tikunov et al. 2005), XCMS (Smith et al. 2006), mzMine (Katajamaa and Oresic 2005), all of them demanding universal netCDF and mzXML file formats.

In metabolomic experiments data underlie biological as well as non-biological (technical) variations. A high number of replicates or samples from pooled material of several plants can lower the biological variations. In order to be able to compare the metabolite levels, relative quantification of detector-response signals requires normalization of signal intensities. Technical variation can be compensated by the use of internal standards and is generally acceptably low, but the optimal normalization of biological variance in plant metabolomic studies remains to be developed. Traditional normalization schemes include normalization to the total ion current (total chromatogram area), to the mean peak intensity (the averaged chromatogram area), or to the most abundant peak intensity as performed in urinary metabolomic approaches (Kind et al. 2007; Wagner et al. 2007). These traditional methods do not consider the effect of changes in metabolite concentrations on the normalization factor, which are of major concern in comparative experiments. Normalization methods that rescale the values based on the data itself are known from the bioinformatics of transcriptomics and proteomics.

Sophisticated statistical tools are being developed to decipher the vast amounts of data and to detect significant differences on the metabolite level that lead to discrimination between samples. Cluster analysis (like hierarchical clustering or principle component analysis) is routinely used for pattern recognition to evaluate and visualize similarities and differences between data sets (Fiehn et al. 2000). In a different approach, pair-wise analysis of linear correlations are used to detect connections between metabolites, and further between 
metabolites, genes, and proteins which directly reveal biochemical pathways or facilitate the identification of unknown metabolic pathways (Steuer et al. 2003). Such statistical tools allow biologically meaningful conclusions to be made from metabolite studies without characterizing the source of every analytical signal (for instance in studying the metabolite diversity of sesame cultivars - see chapter 3 ).

\section{Application of metabolomics in biology and agricultural research}

Plant metabolomic studies have a broad range of application which is reflected by the variety of relevant scientific publications. Metabolic approaches are being used for practical applications, e.g. for monitoring crop quality features (Hall et al. 2005) and in basic research to answer questions varying from metabolic differences between genotypes, to the influence of environmental or genetic manipulations on the metabolite profile which can help to understand changes in growth and plant physiology. For instance, the latter approach has been exploited when unexpected differences between transgenic and wild type plants were detected by nontargeted metabolic profiling (Roessner et al. 2001). Metabolomic approaches have also been proved to be a powerful tool in the search for naturally stress tolerant species and cultivars by characterization of metabolic adaptations in response to various abiotic stresses as demonstrated in many investigations: e.g. metabolic responses to temperature stress in A. thaliana (Kaplan et al. 2004), to salt stress on crops such as rice or tomato (Johnson et al. 2003; Sanchez et al. 2008), nutrient deficiency and toxicity of heavy metals. Investigations of nutrient deficiency addressed a wide range of inorganic nutrients, for instance the impact of the availability of inorganic nitrogen or sulphur, on the metabolite levels (Urbanczyk-Wochniak and Fernie 2005; Nikiforova et al. 2005) and, more recently, on the effect of phosphate deficiency in common bean (Hernández et al. 2007) and barley (Huang et al. 2008). Furthermore, the combination of mRNA transcript and metabolic profiling provides data for genetic engineering of stress resistance in plants with enhanced ability to adapt to low phosphate environments (Hernández et al. 2007). In this context, metabolic profiling of plant root exudates is an attempt to elucidate the phosphorus acquisition mechanism, but unbiased metabolic profiling of root exudates has rarely been reported in this context. In one metabolomic approach, GC-MS and 2-D NMR were used for profiling root exudates of 
barley and wheat in response to cadmium and iron deprivation revealing metal ion ligands, phytosiderophores, and mugineic acids (Fan et al. 2001).

In a few studies metabolic profiling and metabolic fingerprinting have been used to investigate species diversity (Bino et al. 2005; Overy et al. 2005; Schauer et al. 2005). The elucidation of metabolite diversity of cultivars in relationship to genomic data possesses potential for the improvement of crop breeding, since the quality of crop plants is determined to a large extent by their metabolite content (Memelink 2005) which affects their commercial value regarding flavour, fragrance, shelf life, physical attributes, stress and disease resistance (Hall 2006). For example, differences in fruit metabolite composition with antioxidant or photoprotective activities were identified in lighthypersensitive tomato mutants by metabolic profiling (Bino et al. 2005).

Metabolomics can be used also to screen breeding progeny as a tool for the selection of agriculturally important traits. (Overy et al. 2005; Schauer et al. 2005).

In contrast to many studies focussed on physiological development of plant tissue in response to abiotic or elicitor induced stress, very little is reported about applications of nontargeted metabolomic approaches addressed to plant-pathogen interaction studies. Indeed, this is "an even greater challenge" (Allwood et al. 2008) because plant-host interactions generate extremely heterogeneous and biochemically complex samples consisting of a mixture of pathogen-colonised and non-challenged plant cells which further complicate the identification of the origin of metabolites (Allwood et al. 2008). However, metabolomic approaches revealed metabolite changes occurring during interactions of Magnaporthe grisea with Brachypodium distachyon which are supposed to play a role in suppression of defense mechanisms or developing disease symptoms (Allwood et al. 2006). NMR-based metabolomics were used as a tool to investigate the metabolic change of Brassica rapa in response to different typically food borne bacteria (Jahangir et al. 2008). Bednarek et al. (2005) studied differential aromatic metabolite profiles in extracts obtained from A. thaliana roots in response to the root-pathogenic oomycete Pythium sylvaticum.

\section{Phytohormone analysis}

Phytohormones can be described as chemical transmitters of developmental and environmental impulses in plants. Although they are present only in trace amounts in plant 
tissue, phytohormones exhibit crucial signaling effects coordinating and regulating plant growth and eliciting physiological reactions. Changes in concentration of a phytohormone in response to a stimulus also alter hormonal balance, since phytohormones interact with each other. Phytohormones are also known to play an important role in plant defense.

Nine classes of phytohormones are known, which can be divided into i) volatile and ii) non-volatile metabolites: i) ethylene, gibberellins, cytokinins, brassinosteroids, and polyamines, ii) auxins, salicylates, abscisates, and jasmonates. Since metabolic profiling does not compensate for discrimination of compounds present in trace amounts, targeted analyses of phytohormones are additionally required to answer various biological questions, for instance in relation to plant defense reactions. Today, phytohormone analyses are commonly performed in reproducible and reliable ways by using chromatography (GC or LC) hyphenated with sensitive MS detection techniques. Simultaneous detection of multiple components in a single analysis with the use of isotopelabeled standards nowadays represents the standard procedure. Single-targeted phytohormone and profiling methods using GC-MS are well established (Mueller et al. 2002; Schmelz et al. 2003), but they demand the derivatization of non-volatile phytohormones to increase their volatility. This is a critical drawback of GC-based phytohormone analysis, because some phytohormones decompose under the conditions of derivatization or evaporation. Important for quantification using deuterium-labeled standard compounds is that the labeling must survive all steps of analysis. For instance, deuterium -labeling can also get lost during derivatization. Thus, liquid-chromatography coupled to mass spectrometry was introduced in phytohormone analysis and many methods for simultaneous determination of multiple phytohormones in plant extracts have been established (Chiwocha et al. 2003; Durgbanshi et al. 2005). The major challenge in phytohormone analysis is the detection of trace amounts of analytes in a highly complex matrix, available only in small amounts of plant tissue, and concurrently the desire for less laborious sample preparation. In order to increase the sensitivity of detection, modern highly selective detection modes are used in phytohormone analysis, such as selective ion monitoring (SIM), tandem-MS (MS/MS), or multiple reaction monitoring (MRM) techniques (Mueller et al. 2002; Chiwocha et al. 2003; Pan et al. 2008). 


\section{The pathosystem Verticillium longisporum/Brassica spp.}

Verticillium spp. are soil-borne phytopathogens causing vascular diseases in many crop species in temperate and sub-tropical areas (Fradin and Thomma 2006). Verticillium longisporum is a soil-borne vascular fungus responsible for Verticillium wilt on Brassica napus L. spp. oleifera (oilseed rape; Karapapa et al. 1997; Zeise and von Tiedemann 2001, 2002; Steventon et al. 2002) which is the most important oilseed crop. This disease represents a growing threat to oilseed rape production in Europe (causing serious yield losses) due to expanding rapeseed cultivation.

Based on morphological, physiological and molecular differences Karapapa et al. (1997) proposed the controversial distinction between V. dahliae and V. longisporum (firstly classified as a variant to Verticillium dahliae; Stark 1961) as two individual species. They differ further in their host range: V. longisporum has a host range limited to Brassica spp. (Zeise and von Tiedemann 2002), whereas its closely related species V. dahliae cover a wide host range including many economically important crops and trees e.g. tomato, cotton, sunflower (Pegg and Brady 2002a), but not to Brassicaceae. Despite the name Verticillium wilt, the lack of wilting symptoms is a distinctive feature of $V$. longisporum infection of Brassicaceae.

The disease cycles of V. longisporum and V. dahliae are similar (Zhou et al. 2006; Johansson et al. 2006), starting with the germination of soil-borne resting structures (microsclerotia) stimulated by root exudates of the host plant (Mol and Scholte 1995). After penetration and invasion of the root cortex, fungal hyphae enter the vascular tissue where conidia are formed. The colonization of the plant proceeds as conidiospores are distributed upwards within the xylem vessels via the transpiration stream (Beckman 1987, Gold et al. 1996). For most of its life cycle V. longisporum remains confined to individual xylem vessel filled with mycelium, instead of colonizing the vascular system entirely (Eynck et al. 2007). This is supposed to explain why B. napus infected with $V$. longisporum does not cause any wilting symptoms as opposed to $V$. dahliae which is non-pathogenic on B. napus (Eynck et al. 2007). At later stages of the disease, when the diseased plant starts to senesce, the fungus invades non-vascular tissue and develops microsclerotia on the dying stem and leaf tissue (Mol and Scholte 1995) whereby the pathogen can persist in soil for many years (Wilhelm 1955; Schnathorst 1981). 
$V$. longisporum induces the early onset of senescence, premature flowering and stunting of the plant by yet unknown signal(s). The infection on oilseed rape becomes apparent only in the ripening period, when black microsclerotia are formed in stem tissue and emerge from the stem epidermis.

Due to the vascular limitation of Verticillium-Brassicaceae interaction, chemical control of the pathogen by fungicide application is impossible. Therefore Brassica cultivars are intensively investigated for resistance towards $V$. longisporum, but no resistant genotypes are available yet. Accessions of B. oleracea and B. rapa with enhanced resistance have been identified recently (Happstadius et al. 2003; Dixelius et al. 2005; Rygulla et al. 2007). This situation motivates the elucidation of the mechanisms of pathogenicity which may lead to alternative, innovative strategies for disease control.

\section{Chemical signals in $V$. longisporum/B. napus interaction}

\section{Putative roles of secondary metabolites of $\mathrm{V}$. longisporum}

In the interactions between soil-borne vascular fungi and plants the mechanisms of pathogenicity implicates the exchange of chemical signals within the vascular tissue. In the interaction between $V$. longisporum and $B$. napus, both the pathogen and the host plant are supposed to release metabolites into the xylem which affect the other partner.

The symptoms caused by the infection indicate that secondary metabolites secreted by the pathogen play a key role in this infection, acting as virulence factors with phytotoxic, elicitor-like and phytohormonal effects, or as suppressors of plant defense, as it has been reported for other Verticillium spp.. In several studies it has been suggested that Verticillium spp. produces a broad spectrum of phytotoxins and elicitor compounds which induce pathogen-specific symptoms in the absence of the fungus (Nachmias et al. 1987; Buchner et al. 1989; Meyer et al. 1994; Mansoori et al. 1995). In the 1960s, phytotoxins produced by $V$. dahliae and $V$. albo-atrum are reported to cause host cell death (Pegg 1965). A protein-lipopolysaccharide (PLP) complex isolated from crude extracts of $V$. dahliae (Krasilnikov et al. 1969) was proposed to induce wilting symptoms in cotton plants (Keen et al. 1972). Its phytotoxic activity is attributed to a low-molecular-weight glycopeptide (Buchner et al. 1982; Nachmias et al. 1985). A glycoprotein elicitor purified from $V$. dahliae culture fluid was found to trigger the synthesis of phytoalexins and 
oxidative burst (Davis et al. 1998). Recently, VdNep (belonging to Nep1-like proteins) was isolated from $V$. dahliae and suggested to play a role as elicitor (Wang et al. 2004).

Verticillium spp. utilizes cell-wall-degrading enzymes for systemic host colonization (Durrands and Cooper, 1988). Particularly pectinolytic enzymes have been shown to be important for fungal virulence on the host (Carder et al. 1987) enabling Verticillium spp. to overcome pectin-containing pit membranes between xylem elements and at vessel ends (Pegg et al. 1976; Bishop and Cooper 1983).

Disease phenotypes like stunting and premature senescence indicates interference of normal phytohormone function in relation to systemic infection and spread of the fungus (Pegg and Brady 2002b). The elevation of ethylene production after Verticillium infection (Pegg and Cronshaw 1976) is associated with the development of disease symptoms and was found to be involved in disease resistance of $A$. thaliana (Veronese et al. 2003; Tjamos et al. 2005; Johansson et al. 2006). Elicitation of ethylene was referred to Verticillium phytotoxins (Mansoori and Smith 2005). Another group of potential virulence factors represent suppressors of the host defense. In tomato cultivars infected with V. albo-atrum or $V$. dahliae, the pathogen can suppress the activity of phenylalanine ammonia-lyase (PAL) (Lee et al. 1992; Gold and Robb 1995), an essential enzyme which is involved in the synthesis of suberin and lignin (Hahlbrock and Scheel 1989) but also regulates synthesis of salicylic acid (SA). Suppression of PAL activity results in less suberin coating in the xylem which is one part of the plant defense response (Lee et al. 1992).

The host specificity of many fungi is often determined by whether or not the pathogen has the enzymes to detoxify a particular plant defense product (van Etten et al. 1989; Osbourn 1999). As other phytopathogenic fungi infecting Brassica spp., V. longisporum is likely to produce detoxification enzyme(s) (Pedras and Ahiahonu 2005) or transporters (Del Sorbo et al. 2000) as protection against fungitoxic effects of plant defense products. It is unknown whether $V$. longisporum possesses enzymatic activities targeting antimicrobial compounds of crucifers, and thereby determine its host specificity.

\section{Plant defense reactions after Verticillium infection}

Disease resistance of plants generally involves two steps of chemical exchange with the pathogen: firstly recognition through the perception of pathogen-derived signals and subsequently defense reactions (Hahlbrock et al. 2003). 
The host plant reacts against Verticillium attack with the interplay between antimicrobial and physical defense responses. Infected plants show accumulation of antimicrobial components such as phenolics in vicinal tissue, phytoalexins and pathogenesis-related proteins on the one hand (Bell 1969; Cooper et al. 1996; Daayf et al. 1997), barrierforming structures like suberin coating of xylem vessels, tylosis formation and callose depositon on the other (Benhamou 1995; Gold and Robb 1995). Accumulation of elemental sulphur in resistant tomato and cotton cultivars after Verticillium attack was found to contribute to fungal elimination (Williams et al. 2002).

Concerning signaling defense molecules, it has been reported that abscisic acid (ABA) and salicylic acid (SA) associated pathways, but not jasmonic acid (JA) are affected in A. thaliana responses to $V$. longisporum (Veronese et al. 2003; Johansson et al. 2006). In contrast, JA-insensitive $A$. thaliana plants show more susceptibility to $V$. dahliae (Thaler et al. 2004; Tjamos et al. 2005).

Crucifers are known to produce a range of well-characterized secondary metabolites (phytoalexins and phytoanticipins) as a means of protection against pathogens (Pedras et al. 2000, Pedras et al. 2006), but their role in V. longisporum-infection has to be elucidated. Brassicaceae synthesize two groups of sulphur-containing indole derived defense compounds: Phytoalexins and glucosinolates (Pedras et al. 2002). They were isolated from leaves or roots but nothing is known about their occurrence in xylem sap. Most crucifer phytoalexins are biosynthesized from tryptophan. The phytoalexin camalexin represents a resistance factor to fungal infection of A. thaliana (Thomma et al. 1999). Brassica pathogens are specialized in detoxifying phytoalexins. Their detoxification mechanisms and intermediates are well elucidated, involving glucosylation and/or degradation to harmless products. (Pedras and Ahiahonu 2005). Apart from detoxification, Pedras et al. (2008) recently suggested the redirection of the phytoalexin biosynthesis pathway towards less inhibitory compounds caused by the pathogen to avoid accumulation of more effective phytoalexins. Interestingly, Rhizoctonia solani is able to transform camalexin (Pedras and Khan 1997), but not Leptosphaeria maculans and Alternaria brassicicola (Pedras et al. 1998). Camalexin-deficient mutants of A. thaliana do not show any changed response to $V$. longisporum infection (Johansson et al. 2006), which indicate that $V$. longisporum might have capacities either to tolerate or detoxify camalexin.

Glucosinolates were found to be involved in defense responses of cruciferous plants (Fahey et al. 2001; Pedras et al. 2008). They are hydrolyzed by myrinases to fungitoxic 
isothiocyanate derivatives (Tierens et al. 2001). Some fungi can tolerate these cyanogenic products by enzymatic detoxification involving cyanide hydratase (Fry and Myers 1981) or glutathione transferase (Sellam et al. 2006), among Brassicaceae-infecting fungi, Leptosphaeria maculans (Sexton and Howlet 2000) and Alternaria brassicicola (Cramer and Lawrence 2004; Sellam et al. 2006).

Recently, it was found that polar metabolites, such as polar indole metabolites (Hahlbrock et al. 2003), soluble compounds (Hagemeier et al. 2001), cell-wall bound phenolics (Tan et al. 2004) and glucosyl indole-3-carboxylic acids (Bednarek et al. 2005), and tryptophan (Pedras et al. 2008) are induced in Brassicaceae defense.

\section{Aims of the work}

The aim of the PhD project was to develop experimental procedures and data processing routines suitable for the application of nontargeted metabolic profiling to different kinds of comparative experiments in plant systems. An important goal was the assessment and adjustment of software tools for data processing (noise reduction, chromatogram alignment, peak matching, normalization of intensities and comparative analysis) and the application of the system to the analysis of xylem sap of Brassica napus infected with Verticillium longisporum.

A further goal of this work was to test the suitability of the developed procedures on other plant systems investigated in the department. In the course of the work, the developed procedure for nontargeted metabolic profiling was applied to samples generated by colleagues in the department working on two other projects: (i) the role of root exudates of sugar beet in phosphorus mobilization (Prof. Claasen's group, samples generated by Dr. Reza Khorassani), and (ii) metabolic diversity in sesame (Prof. Karlovsky's group, samples generated by Dr. Hernán Laurentin). 


\title{
Chapter 2: Differential metabolic profiling of xylem sap of Brassica napus infected with Verticillium longisporum *
}

*This manuscript is prepared for publication.

\begin{abstract}
Astrid Ratzinger, ${ }^{1}$ Nadine Riediger, ${ }^{2}$ Andreas von Tiedemann, ${ }^{2}$ Petr Karlovsky ${ }^{1}$
${ }^{1}$ Molecular Phytopathology and Mycotoxin Research Unit \& ${ }^{2}$ Plant Pathology and Plant Protection Unit, Department of Crop Sciences, Goettingen University, Grisebachstrasse 6, 37077 Goettingen, Germany
\end{abstract}

\begin{abstract}
Verticillium longisporum is a vascular pathogen infecting plants of the Brassicaceae family. Because symptoms in the shoot occur while the pathogen is still restricted to the xylem vessels in the roots, signals translocated upward within the plant are supposed to be involved in the etiology of the disease. To identify these signals, we performed nontargeted metabolic profiling of xylem sap extracted from healthy Brassica napus plants and from plants infected with $V$. longisporum. HPLC-MS profiles were processed by XCMS, an open source software tool for peak matching and retention time alignment, and subjected to comparative analysis. Metabolic signals with levels differing between healthy and infected plants were identified and verified on xylem sap extracted from single plants. Nineteen metabolites affected by the infection were found, belonging to three classes: metabolites enhanced by infection, suppressed by infection and infection-specific metabolites (the latter metabolites never occurred in healthy or $V$. dahliae-infected plants). Structural characterization of infection-specific signals by mass spectrometric fragmentation revealed common building blocks derived from indole such as indole carboxylic acid, tryptophan and indole pyruvate. The levels of infection-specific signals within different xylem sap pools were strongly correlated. Changes in the concentration of xylem metabolites affected by infection were monitored and the correlation with diseases symptoms and fungal biomass were investigated.
\end{abstract}


Additional keywords:

Brassica napus, Verticillium longisporum, HPLC-MS, XCMS, metabolic profiling, xylem sap, indole derivatives
Abbreviations:
amu atomic mass unit,
dpi days post inoculation,
MS mass spectrometry,
$\mathrm{m} / \mathrm{z} \quad$ mass-to-charge ratio

\section{(Introductory statements)}

Verticillium longisporum is a soil-borne phytopathogenic fungus adapted to crucifers (Brassicaceae). As a typical vascular pathogen, V. longisporum enters the host via roots and remains restricted to xylem vessels of the root system for most of its infection period, while the shoots develop disease symptoms (Eynck et al. 2007; Zhou et al. 2006). The symptoms caused by the infection, like stunting and premature ripening, indicate that secondary metabolites might play an important role in the interaction between phytopathogenic Verticillium species and host plants. Both the pathogen and its host plant are expected to synthesize substances which affect the other partner. Crucifers are known to produce a range of well-characterized phytoalexins as a response and phytoanticipins as a protection against bacteria and fungi (Pedras et al. 2000). Secondary metabolites of Verticillium spp. are believed to play a key role in the pathogenicity, acting as virulence factors with phytotoxic, phytohormonal or defense-suppressing effects (Fradin and Thomma 2006). While the cytology of infection and genetics of the resistance to $V$. longisporum infection in B. napus and Arabidopsis thaliana have been addressed in the last decade (Veronese et al 2003, Zhou et al. 2006, Johansson et al 2006, Eynck et al. 2007), no recent studies focused on small molecules involved in the interaction.

The application of nontargeted metabolic profiling is particularly useful in this context, because it includes the detection of previously unknown metabolites. In contrast to many studies focused on physiological development of plant tissue in response to abiotic or elicitor induced stress, very little is reported about applications of nontargeted 
metabolomic approaches addressed to plant-pathogen interaction studies. This is no easy challenge because plant-host interactions involve extremely heterogeneous and biochemically complex samples consisting of a mixture of pathogen-colonised and nonchallenged plant cells which further complicate the identification of the origin of metabolites (Allwood et al. 2008). However, metabolomic approaches revealed metabolite changes occurring during interactions of Magnaporthe grisea with Brachypodium distachyon which are supposed to play a role in suppression of defence mechanisms or developing disease symptoms (Allwood et al. 2006). NMR-based metabolomics were used as a tool to investigate the metabolic change of Brassica in response to different typically food borne bacteria (Jahangir et al. 2008). Bednarek et al. (2005) studied differential aromatic metabolite profiles in extracts obtained from Arabidopsis roots in response a rootpathogenic oomycete Pythium sylvaticum. Recently, Pedras et al. (2008) investigated defense metabolite profiles in leaves of B. rapa L. species oilseed canola and rapeseed and observed differential metabolic responses in compatible and incompatible interaction with virulent and avirulent races of the biotroph Albugo candida.

HPLC with ESI-MS detection is the method of choice for covering a wide range of small molecules of medium to high polarity with mass of up to $1000 \mathrm{Da}$. For peak matching and retention time alignment in metabolic data generated by HPLC-MS, new open source software XCMS (Smith et al. 2006) is suitable for peak matching and retention time alignment in metabolic profiling. Its application has so far been reported in medical studies and research on mammals (Kind et al. 2007; Wikoff et al. 2007; Dunn et al. 2008). XCMS has so far not been applied to plant metabolomics.

The objective of this work was to identify xylem sap metabolites affected by interaction with $V$. longisporum and to determine their origin, using HPLC-MS for the analysis and XCMS for data processing.

\section{RESULTS}

\section{Nontargeted differential metabolic profiling of pooled xylem sap samples}

The specific feature of Verticillium-Brassica pathosystem is that chemical interactions between plant and fungus are confined to the vascular tissue. Xylem sap of B. napus plants infected with $V$. longisporum and healthy control plants was analyzed by RP-LC-MS in 
order to identify metabolites affected by the infection. At 28 days past infection (dpi) the plants were divided into three groups by height. Each group consisted of four plants. Xylem sap was collected and pooled for each group, generate three samples. In order to prevent selective losses of specific metabolites during cleanup, raw xylem sap was filtered and directly subjected to RP-LC-MS analysis.

For the ionization of substances separated by HPLC we used electrospray (a soft ionization technique) in order to generate molecule ions. Metabolite profiles were acquired on an ion trap mass spectrometer in full-scan mode. Metabolic profiles in form of three-dimensional data (retention time - $\mathrm{m} / \mathrm{z}$ value - intensity) were processed by a routine consisting of (i) peak detection, chromatogram alignment and peak integration across samples by XCMS (Smith et al 2006), (ii) trimmed-mean normalization of ranked intensity ratios (iii), and comparative analysis.

A total of 93 peaks in negative ESI mode and 146 peaks in positive ESI mode were detected in xylem sap profiles after processing with XCMS (Smith et al. 2006). For the comparative analysis, the ratios of normalized areas for each peak in infected and healthy plants were calculated and used to identify signals affected by infection. Mass signals originated from isotopomers containing ${ }^{13} \mathrm{C}$ and obvious solvent and alkali metal adducts were eliminated.

By comparative analysis 19 signals with intensities affected by infection were identified. Two kinds of changes in signal intensities (metabolite levels) in infected samples as compared to healthy were observed: (i) signals enhanced or reduced by infection (appearing in both infected and control plants), and (ii) infection-specific signals (present only in the diseased plants). Signals with intensity ratios higher than 3 (infected versus control plants) were labeled as induced and signals with ratios below 0.4 as suppressed. We found no signals occurring in healthy plants and missing in infected plants. A total of 19 MS signals affected by infection were identified.

The strongest increase of a signal after infection was 300 -fold. Inspection of the raw data revealed that many peaks with intensity ratios larger than 10 were in fact missing in the controls. The areas used for the calculation of the increase factor resulted from peak integration performed by XCMS, which often confused noise (background) for signals. We manually assigned the induction factors of these signals to infinite. The results are summarized in Tab. 1. 
Comparison of normalized signal intensities revealed that all 8 infection-specific signals correlated with disease severity. As shown in Fig. 1, we scaled the intensities to the first sample group. The re-scaling revealed a common pattern: In sample B, each signal reached $21 \pm 5 \%$ of the intensity of the corresponding signal in sample $\mathrm{A}$, while signals in pool $\mathrm{C}$ reached $39 \pm 6 \%$ of their intensities in sample A. This pattern was only observed among infection-specific signals.

A similar but less pronounced relationship among the intensities of suppressed signals only existed for two signals (data not shown). Among the enhanced signals, only $\mathrm{m} / \mathrm{z} 187$ showed an intensity pattern (1: $0.19: 0.59)$ similar to the infection-specific signals as described for infection-specific signals.

\section{Analysis of xylem sap from individual plants infected with $V$. longisporum and V. dahliae}

The severity of disease symptoms after $V$. longisporum infection varies considerably from plant to plant in B. napus (Eynck et al. 2007) and A. thaliana (Tappe et al. 2008). As we observed a relationship between the intensity of infection-specific signals and disease phenotype with pooled xylem sap samples (see above), we also analyzed xylem sap from individual plants. The yield of xylem sap from a single plant at 28 dpi allowed for several HPLC injections. The analysis was conducted in selective ion monitoring mode in order to enhance the sensitivity for candidate ions. Because normalization based on full-scan MS data was not possible, simultaneous photometric detection was performed in a diode array detector and this data were used for the normalization.

In addition to $V$. longisporum, $V$. dahliae-infected plants were included in this experiment in order to find out whether the metabolic changes detected after $V$. longisporum-infection were species-specific.

All signals listed in Tab. 1, which were previously found in pooled xylem sap to be affected by infection, were also found in xylem samples from single plants and were affected by infection with $V$. longisporum in the same way. Strong correlations of intensities of infection-specific signals $\mathrm{m} / \mathrm{z} 396$ versus $\mathrm{m} / \mathrm{z} 558$ and $\mathrm{m} / \mathrm{z} 611$ versus $\mathrm{m} / \mathrm{z} 554$ were observed (Tab. 2). No correlation was found between disease severity (given by plant height reduction) and metabolite intensities (data not shown). 
Signals detected after infection with $V$. longisporum but not in healthy plants were also missing from xylem sap of plants infected with $V$. dahliae. Furthermore, the effects (suppression or increase) of $V$. longisporum on signals present in healthy plants were not observed with $V$. dahliae (Fig. 2b). This is in line with disease symptoms, which were only observed in $V$. longisporum infection. Infection with $V$. dahliae did not cause any stunting (Fig 2a).

\section{Changes in metabolite levels and fungal DNA content in the course of infection}

Because the metabolites affected by infection were originally categorized as suppressed, enhanced and infection-specific based on their intensities at $28 \mathrm{dpi}$, the question arose whether this assignment holds for other time points. Concerning the infection-specific metabolites, we were interested to learn whether they occur already at an early stage of infection and how these changes are related to the colonization of plant tissue by the fungus.

Xylem sap was collected from plants at 14, 21, 28, and 35 dpi after infection with $V$. longisporum and the intensities of selected metabolic signals were quantified. Because the yield of xylem sap delivered by a single plant at early time points $(14,21 \mathrm{dpi})$ was low, samples for analysis were prepared by pooling xylem sap from 4 plants for each sample.

The results for four selected metabolites are shown in Fig. 3. Some signals were present from 14 dpi on while others appear only at 28 dpi. The intensity of each signal followed one of the following time-course patterns.

Metabolic signals with $\mathrm{m} / \mathrm{z} 611,177,554,527,470,396$, and 449 were present in xylem sap of infected plants already at 14 dpi and their intensity continuously increased till $28 \mathrm{dpi}$; their intensity remained unchanged between 28 dpi and 35 dpi with the exception of signal $\mathrm{m} / \mathrm{z} 192$ which strongly decreased during the last phase of infection. Metabolites 137,187 , and 351 were present already at 14 dpi and remain at the same level during the whole experiment with the exception of signal $\mathrm{m} / \mathrm{z} 299$ which slightly decreases its level with time. Metabolite 558, 476, 617, and 655 were low at 14 dpi and 21 dpi and increased from $21 \mathrm{dpi}$ to $35 \mathrm{dpi}$.

DNA levels in hypocotyls and leaves during the infection are shown in Fig 4. In the hypocotyls the amount of fungal DNA continuously increased over all time points. In leaves, $V$. longisporum DNA was detectable only in low amounts at early time points and 
increased strongly between 21 and $28 \mathrm{dpi}$. The development of fungal DNA in leaves parallels the increase of signal intensities for $m / z 558,476,617$, and 655.

\section{Correlations between intensities of infection-specific signals}

Xylem sap pools obtained from 4 plants at 14, 21, 28, and 35 dpi after infection with $V$. longisporum were used to investigate the relationship between intensities of infectionspecific signals by pair-wise comparison. While strong correlations of signal intensities among 6 individual infected plants were found only for two pairs of metabolites (see above), strong metabolite-metabolite correlation for all signals in xylem sap pools were found (Tab 2.). Three of them are presented in Fig. 5.

\section{Characterization of infection-specific metabolic signals}

In xylem sap from B. napus, 8 signals only occurred after infection with $V$. longisporum. Even after 20-times concentration of xylem sap extracts infection-specific signals were not detected in control samples, they indeed only occur in xylem sap. These signals were analyzed in depth by $\mathrm{MS}^{\mathrm{n}}$ fragmentation on an ion trap.

Data-depending fragmentation and product ion scan of these signals revealed that all were structurally related in the following way (see Fig. 6). Four of the signals detected in positive ionization mode possessed indole-3-carboxylate moiety as a common building block (signals $[\mathrm{M}+\mathrm{H}]^{+} \mathrm{m} / \mathrm{z}$ 613, 556, 470, and 527). These signals eluted between 7 and 11 min. Two of these compounds $\left(\mathrm{m} / \mathrm{z} 613\right.$ and 556) formed an ion of $\mathrm{m} / \mathrm{z} 410$ by $\mathrm{MS}^{2}$ fragmentation, whereas the other two compounds ( $\mathrm{m} / \mathrm{z} 470$ and 527) led to a daughter ion of $\mathrm{m} / \mathrm{z}$ 324. Furthermore, metabolites $\mathrm{m} / \mathrm{z} 613$ and $\mathrm{m} / \mathrm{z} 527$ shared a neutral loss of 203 amu, while metabolites $\mathrm{m} / \mathrm{z} 556$ and $\mathrm{m} / \mathrm{z} 470$ shared a neutral loss of $146 \mathrm{amu}$. Further relationship was revealed by $\mathrm{MS}^{3}$ fragmentation of $\mathrm{MS}^{2}$ product ions $\mathrm{m} / \mathrm{z} 410$ and 324 . Both ions yielded the same product ion $\mathrm{m} / \mathrm{z}$ 162, which was likely protonated indole-3carboxylic acid.

Both molecules yielding $[\mathrm{M}+\mathrm{H}]^{+} \mathrm{m} / \mathrm{z} 613$ and $[\mathrm{M}+\mathrm{H}]^{+} \mathrm{m} / \mathrm{z} 556$ in positive mode formed ions in negative ESI mode, too, leading to $[\mathrm{M}-\mathrm{H}]^{-} \mathrm{m} / \mathrm{z} 611$ and $[\mathrm{M}-\mathrm{H}]^{-} \mathrm{m} / \mathrm{z} 554 \mathrm{MS}^{3}$ fragmentation of the daughter ion $\mathrm{m} / \mathrm{z} 410$ in positive mode implied a neutral loss of $248 \mathrm{amu}$. In negative mode (Fig. 7), the total neutral loss of 248 amu occurred in two steps. $44 \mathrm{amu}$ were eliminated in $\mathrm{MS}^{2}$ and further $204 \mathrm{amu}$ in $\mathrm{MS}^{3}$, yielding $\mathrm{m} / \mathrm{z} 306$ from 
$\mathrm{m} / \mathrm{z} 554$ and $\mathrm{m} / \mathrm{z} 363$ from $\mathrm{m} / \mathrm{z}$ 611. In another fragmentation path a neutral loss of $409 \mathrm{amu}$ occurs from both signals $(\mathrm{m} / \mathrm{z} 611, \mathrm{~m} / \mathrm{z} 554)$. In contrast to molecular ions $[\mathrm{M}+\mathrm{H}]^{+} \mathrm{m} / \mathrm{z} 613$ and $[\mathrm{M}+\mathrm{H}]^{+} \mathrm{m} / \mathrm{z} 556$, no corresponding negative molecular ions were observed for $[\mathrm{M}+\mathrm{H}]^{+}$ $\mathrm{m} / \mathrm{z} 470$ and $[\mathrm{M}+\mathrm{H}]^{+} \mathrm{m} / \mathrm{z} 527$.

Two infection-specific signals were only observed in negative ionization mode: $\mathrm{m} / \mathrm{z} 396$ and $\mathrm{m} / \mathrm{z} 558$. The common feature of their fragmentation, which they share with those two members of the previous group that form negative ions $(\mathrm{m} / \mathrm{z} 611$ and $\mathrm{m} / \mathrm{z} 554)$, is a neutral loss of $204 \mathrm{amu}$.

Extraction of pure xylem sap revealed that none of the infection-specific metabolites was extractable into ethyl acetate. Subsequent partition between xylem sap and n-butanol yielded the following ratios (butanol : watery phase): 50:50 for $\mathrm{m} / \mathrm{z} 470,30: 70$ for $\mathrm{m} / \mathrm{z} 527$, 10:90 for $\mathrm{m} / \mathrm{z} 556 / 554,613 / 611,558,0: 100$ for $\mathrm{m} / \mathrm{z} \mathrm{396}$. The order of the elution from polar modified reverse-phase column contradicts this rank partially: $m / z 527<m / z 470<$ $m / z 611 / 613<m / z 558, m / z$ 554/556, < m/z 396.

The metabolic signals specific for xylem sap of $V$. longisporum-infected plants were searched in the shoot of infected plants. Neutral, acidic and basic methanol/chloroform/water extracts were prepared from leaves and stems of the plants from which xylem sap originated. All infection-specific signals were detected in methanol phase of neutral and acidic extracts.

None of the infection-specific signals found in xylem sap was detected in culture supernatants of pure $V$. longisporum cultures in xylem sap-simulating medium (Neumann and Dobinson 2003).

\section{DISCUSSION}

Xylem sap is the primary target for metabolic analysis of the interaction of vascular pathogens with plants. Reverse-phase LC was chosen in our work because we were interested in secondary metabolites rather than sugars, amino acids and inorganic ions, which are well-known components of xylem sap. The purpose of the comparative analysis of metabolic profiles was to find differences in the constitution of xylem sap between infected and healthy plants. For peak detection and retention time alignment we used an 
open source software XCMS (Smith et al. 2006), which was used first in metabolic analysis of knockout mice and human plasma (Nordström et al. 2006) and in a search for cancer biomarkers in urine (Kind et al. 2007) but has not been applied to plant metabolomics so far. Our results show that XCMS is principally suitable to analyze nontargeted metabolic profiles of xylem sap, but signals identified as varying between controls and treatments need to be re-checked manually. For signals occurring in the treated samples but not in the controls, XCMS attempts to integrate the corresponding area of the chromatogram in the control group, generating false intensities (integrated noise) and which lead to wrong "induction factor" calculation. This typically happened with infection-specific metabolites (see Tab 1). It is necessary to correct these values manually; despite this inconvenience, XCMS proved very useful for processing full-scan MS profiles of xylem sap.

Normalization is particularly important for xylem sap data, because the complexity of the sample is moderate and its composition is affected by the variation introduced during xylem sap extraction to a high degree. Varying amounts of water enter xylem during root pressurization in the Scholander bomb, causing additional variability to the underlying biological variability of metabolites. Because of varying dilution of xylem sap which occurs during pressurization, combined with a relatively low number of usable MS signals (80 - 150 per aligned chromatogram) and the unknown effect of infection on the concentration of metabolites underlying these signals, the traditional normalization schemes (largest peak, total ion current, total chromatogram area) are inadequate. We therefore used a normalization scheme developed for quantitative transcript profiling (Venkatesh et al. 2005), which excludes signals likely to be affected by the treatment from the calculation of the normalization coefficient. The analysis of xylem sap extracted from single plants was performed by selective ion monitoring; because full-scan data were not available, we normalized MS signals by the sum of areas of peaks detected by a UV absorption photometer (diode array detector). That some of these peaks were affected by infection can not be excluded but taking into account that most signals affected by infection somewhat increased than decreased, the inclusion of infection-affected signals in the normalization would rather underestimate than overestimate the number and relative increase of signals enhanced by infection.

We found three groups of xylem metabolites signals affected by infection: signal with intensities enhanced or reduced after infection and infection-specific signal. The latter only 
occurred in $V$. longisporum-infected plant, they were not detected in healthy plants nor in plants infected with $V$. dahliae. Signals with intensities reduced after infection might have originated from plant compounds metabolized by $V$. longisporum, possibly defense chemicals known as phytoanticipins. Several fungal pathogens of Brassica spp. are known to enzymatically detoxify phytoanticipins and phytoalexins of their hosts (Pedras et al. 2002; Pedras and Ahiahonu 2005; Pedras et al. 2008). Less common but also possible is that the pathogen blocked a biosynthetic pathway in the plant, inhibiting the production of defense chemicals. Pedras et al. (2008) reported that Albugo candida was able to overcome the defense reponse of $B$. rapa by redirection its phytoalexin biosynthetic pathway towards less detrimental phytoalexin rutalexin. The putative masses of compounds reduced after infection did not correspond to the masses of known defense chemicals of Brassica spp. (Pedras et al. 2006), indicating that xylem sap of B. napus might contain so far unknown phytoanticipins. Alternatively, metabolites suppressed after infection might be precursors of phytoalexins. Purification of these metabolites and determination of their antifungal effects would be necessary in order to distinguish among these hypotheses, while structure elucidation would be needed in order to identify precursors of known phytoalexins. Because compounds enhanced by infection are present in xylem sap from uninfected plants, the material needed for their purification can easily be generated in large amounts. Signals enhanced after infection might originate from phytoalexins. Phytoanticipins are constitutively present in plants, while phytoalexins are synthesized de novo after pathogen attack. The putative masses of these compounds did not correspond to the masses of previously described phytoalexins (Pedras et al. 2006), indicating that xylem of B. napus might contain yet unknown phytoalexins. Detoxification or degradation products of defense compounds might be also enhanced after infection. Alternatively, the infection with $V$. longisporum might have re-directed the synthesis of phytoalexins towards products less harmful to the fungus (Pedras et al. 2008).

Most interesting metabolites occurred in xylem sap of $V$. longisporum-infected plants but not in healthy plants or plants infected with V. dahliae. Because we have not found these signals in culture supernatants of $V$. longisporum (data not shown), our working hypothesis is that they, too, are of plant origin. Their mass-to-charge ratios do not correspond to known phytoalexins (Pedras et al. 2000; Pedras et al. 2006), indicating that the compounds might be previously unknown phytoalexin or products of $V$. longisporum-specific detoxificaton of plant defense chemicals by fungal enzymes. If the latter is the case, this 
detoxification might be involved in the determination of host specificity of $V$. longisporum. Detoxification of defense chemicals and its role in the determination of host range has been investigated in depths for other many fungi infecting Brassicaceae (e.g., Pedras and Ahiahonu 2005) but not for V. longisporum so far. Alternatively, V. longisporum might redirect host's metabolism towards less fungitoxic compounds as mentioned above in context with plant metabolites enhanced by infection.

The intensity of metabolic signals affected by $V$. longisporum did not change after infection with $V$. dahliae. This observation is in line with the lack of disease symptoms in B. napus infected with $V$. dahliae, which is considered non-pathogenic on this plant (Eynck et al. 2007).

As shown in Fig. $3 \mathrm{~A}$ and $3 \mathrm{~B}$, the development of signal intensities over time for two signals occurring only after $V$. longisporum-infection parallels the growth of fungal biomass in the plant tissue (comp. Fig. 4). However, no correlation between the amount of fungal DNA and signal intensities of the metabolites was observed with individual plants at 28 dpi.

A strong metabolite-metabolite correlation was found among infection-specific signals, suggesting that they are derived from the same pathway. The characterization of infectionspecific signals by mass spectrometric fragmentation points in the same direction (Fig. 6 and 7). All infection-specific signals detectable in negative ESI mode show a neutral loss of $204 \mathrm{amu}$, which is likely to be tryptophan. The metabolites with $\mathrm{m} / \mathrm{z} 396$ and $\mathrm{m} / \mathrm{z} 558$ detectable only in negative mode show fragment ions at m/z 192 and m/z 174, respectively, with a difference of $18 \mathrm{amu}$ possibly indicating a hydroxyl group. The fragment ion at $\mathrm{m} / \mathrm{z} 174$ has the same mass as indole-3-acetic acid in negative mode. The cleavage of $44 \mathrm{amu}$, referring to $\mathrm{CO}_{2}$, from signals $\mathrm{m} / \mathrm{z} 611$ and $\mathrm{m} / \mathrm{z} 554$ in the first fragmentation step in negative mode, indicates that they contain a carboxylate moiety. This, enabling stabilization of a negative charge, can maybe explain our finding that both molecules form stabile ions in negative and positive ESI mode $(\mathrm{m} / \mathrm{z} 613$ and $\mathrm{m} / \mathrm{z} 556$ in positive mode), whereas signals $\mathrm{m} / \mathrm{z} 470$ and $\mathrm{m} / \mathrm{z} 537$ can only be detected in positive mode. The latter share a fragment ion at $\mathrm{m} / \mathrm{z} 324$ (positive mode) in the first fragmentation step, leading to $\mathrm{m} / \mathrm{z} 162$ in $\mathrm{MS}^{3}$, and $\mathrm{m} / \mathrm{z} 118$ in $\mathrm{MS}^{4}$. The fragment ion with $\mathrm{m} / \mathrm{z} 324$ in positive mode was identified as $\beta$-D-glucopyranosyl indole-3-carboxylate moiety according to its molecular mass and its fragmentation pathway which is similar to those reported (Bednarek et al. 2005, supplementary material) for B-D-glucopyranosyl indole-3-carboxylic acid with a 
molecular weight of 323. The common $\mathrm{MS}^{3}$ fragment ion at $\mathrm{m} / \mathrm{z} 162$ for all infectionspecific signals detected in positive mode is likely to be an indole-3-carboxylate moiety, and the fragment ion at $\mathrm{m} / \mathrm{z} 118$ can be referred to indole moiety.

Infection-specific metabolites were not extractable from water phase into ethyl acetate or n-butanol, which indicates that they were very polar. Based on mass spectrometric characterization, their extractability and hits in KEGG compound database for molecules with masses corresponding to daughter ions and neutral losses observed in the fragmentation (Fig. 6 and 7), we postulate putative building blocks of infection-specific metabolites signals as shown in Tab. 3. According to this hypothesis, a common motif of all infection-specific metabolites is indole, which occurs one time in metabolite No.1, twice in metabolites No. 2, 3, 5, and 6 and three time in the metabolite No. 4. Tryptophan has been known as building block of phytoalexins and glucosinolates in Brassica spp. and other crucifers, but no metabolite of B. napus containing three indoles has been known so far.

Structures containing three indole moieties appear uncommon, but indole pigments have been recently described as products of a spontaneous reaction between indole-3-pyruvate and tryptophan without any enzymatic activity (Zuther et al. 2008), even though this spontaneous reaction seems to be very unlikely to occur in xylem sap. Indole-3-carboxylic acid glucose esters, among $\beta$-D-glucopyranosyl indole-3-carboxylic acid and 6-hydroxyindole-3-carboxylic acid 6-O- $\beta$-D-glucopyranoside, as well as tryptophan, have been reported to be soluble compounds in leaves of $A$. thaliana, which were induced in defense reaction to Pseudomonas syringae pathovar tomato. Free indole-3-carboxylic acid which was also enhanced in this defense response, but was found only as a cell-wall bound constituent (Hagemeier et al. 2001). This is in line with our result: free indole- 3carboxylic-acid was not detected in xylem sap. The predominance of indole derivates among accumulated metabolites in A. thaliana/ Pseudomonas syringae pathovar tomato interaction was supposed to be relevant in pathogen defense (Hagemeier et al. 2001), for instance cell wall-bound indole-3-carboxylate was assumed to be an end products of pathogen defence-related indolic metabolism.

Our results indicate that indole derivatives play a crucial role in the interaction between $B$. napus and $V$. longisporum in the vascular tissue of infected plants. Infection-specific metabolites detected in xylem sap appear to be novel compounds. Work towards the purification and characterization of these metabolites is under way. 
Metabolite profiling analyses by LC coupled to ESI-MS were initiated to characterize the metabolic signals in B. napus/V. longisporum interaction. This study revealed that the main variation at the metabolite level in xylem sap of B. napus infected with $V$. longisporum, came from the occurrence of several indolic compounds whose accumulation increases during the disease progress. These compounds are specific in B. napus/V. longisporum interaction.

\section{MATERIALS AND METHODS}

\section{Plant material}

Rapid-cycling rape, (Brassica napus var. napus, genome ACaacc) was supplied by Paul H. Williams (Department of Plant Biology, University of Wisconsin-Madison, Crucifer Genetics Cooperative, Stock number 5). Seeds were surface sterilized by immersing them into $70 \%$ ethanol for $30 \mathrm{~s}$. Afterwards seeds were rinsed with sterilized tap water three times for $30 \mathrm{~s}$ and sown in sterile silica sand.

\section{Fungal isolates}

All experiments in this study were carried out using Verticillium longisporum VL 43 from Brassica napus and Verticillium dahliae VD 73 from Linum usitatissimum. Both strains were isolated from plants grown in northern Germany as described in Zeise and von Tiedemann (2001).

Fungal stocks with $1-3 \times 10^{6}$ conidia per $\mathrm{ml}$ were stored at $-80{ }^{\circ} \mathrm{C}$ in $25 \%$ glycerol.

\section{Preparation of inoculum for plant infection}

Shaking cultures were started by adding $500 \mu \mathrm{L}$ of the spore suspension (1-3 x $10^{6}$ conidia per $\left.\mathrm{ml}\right)$ to $300 \mathrm{ml}$ Czapek Dox broth. The inoculated broth was incubated at $23{ }^{\circ} \mathrm{C}$ on a rotary shaker at $100 \mathrm{rpm}$ in the dark. After $10-14$ days, the culture was filtered through a sterile gauze. Spore concentration was determined using a haemocytometer and was adjusted to $1 \times 10^{6}$ spores per $\mathrm{ml}$. 


\section{Plant inoculation and cultivation}

Seedlings were grown in autoclaved silica sand in climate-chambers under constant conditions for seven days $\left(30.0 \mathrm{kLux}, 60 \%\right.$ humidity, $22 / 20{ }^{\circ} \mathrm{C}$ day/night, and 15 -h day length; Philips TL5 HO lamps). Seven-day-old seedlings were carefully taken out of the substrate and inoculated by root-dipping in a spore suspension of either $V$. longisporum (isolate $43,1 \times 10^{6}$ spores per $\mathrm{ml}$ ) or $V$. dahliae (isolate $73,1 \times 10^{6}$ spores per $\mathrm{ml}$ ) for 30 45 minutes. As a control, seedlings were dipped in sterile water. After inoculation, single seedlings were potted in a sterile sand-soil mixture (1:1) and grown under the conditions described above.

\section{Xylem sap preparation}

Plants were harvested 28 days post inoculation (dpi). For time-course experiments, xylem sap was collected 14, 21, 28, and 35 dpi. Shoots were cut above the hypocotyl, and xylem sap was collected by pressurizing roots to $4 \times 10^{5} \mathrm{~Pa}$ for $15 \mathrm{~min}$ using a Scholander pressure chamber. Xylem sap was filtered through a $0.2-\mu \mathrm{m}$-Teflon filter (WICOM, Heppenheim, Germany) and subjected to HPLC-MS analysis immediately or stored at $20^{\circ} \mathrm{C}$. Xylem sap was checked for contamination with cytoplasm by determining malate dehydrogenase activity (absence of activity indicated absence of cytoplasm contamination).

\section{Xylem sap extracts}

$20 \mathrm{ml}$ of xylem sap either obtained from infected or healthy plants was extracted twice with equal volume of ethyl acetate. The watery xylem sap phase was subsequently extracted twice with equal volume of n-butanol. Combined extracts of the same solvent and the remaining xylem sap phase were evaporated to dryness under vacuum at $30^{\circ} \mathrm{C}$. The residue was dissolved in $1 \mathrm{ml}$ of methanol/bi-distilled water (1:9), and the solution was filtered through a $0.2-\mu \mathrm{m}$-Teflon filter. Samples were directly subjected to HPLCmass spectrometry (HPLC-MS) or stored at $-20{ }^{\circ} \mathrm{C}$. 


\section{Preparation of shoot extracts}

The shoots of plants from which xylem sap was obtained were shock frozen with liquid nitrogen and stored at $-80{ }^{\circ} \mathrm{C}$. Frozen shoot material was lyophilized (at $-20{ }^{\circ} \mathrm{C}$ ) for $24 \mathrm{~h}$. Dry shoot material was finely ground in a ball mill. Extraction protocol described by Birkemeyer and Kopka (2007) was modified as follows: to $50 \mathrm{mg}$ of dry shoot material (two replications for each plant), $1.5 \mathrm{ml}$ methanol (gradient quality; Fisher Scientific, Schwerte, Germany) and $1.0 \mathrm{ml}$ chloroform (p.a.; Roth, Karlsruhe, Germany) were added. The mixture was vigorously shaken and incubated for $5 \mathrm{~min}$ at $37^{\circ} \mathrm{C}$. Subsequently, $2 \mathrm{ml}$ of either twice-distilled water, $2 \%$ formic acid (puriss. p.a.; Fluka, Seelze, Germany), or $0.1 \mathrm{M}$ calcium carbonate in water were added. The suspension was shaken for $2 \mathrm{~h}$ at room temperature. The samples were centrifuged at $3583 \mathrm{~g}$ for $15 \mathrm{~min}$, the phases were separated, and each phase was evaporated under vacuum to dryness at $30{ }^{\circ} \mathrm{C}$. The residue of the polar phase was dissolved in $1 \mathrm{ml}$ of methanol/bi-distilled water (1:9), and the solution was filtered through a $0.2-\mu \mathrm{m}$-Teflon filter. Samples were directly subjected to HPLC-mass spectrometry (HPLC-MS) or stored at $-20{ }^{\circ} \mathrm{C}$.

\section{Preparation of culture supernatants from $V$. longisporum}

Fungal cultures were started by adding $100 \mu \mathrm{L}$ of the spore suspension $\left(1-4 \times 10^{6}\right.$ conidia per $\mathrm{ml}$ ) to $100 \mathrm{ml}$ simulated xylem fluid medium (Neumann and Dobinson 2003). The inoculated cultures were incubated at $20^{\circ} \mathrm{C}$ in darkness. After $10-14$ days, the culture was filtered through a paper filter. Culture filtrates were extracted twice with equal volume of ethyl acetate. Subsequently, the watery phase of the medium was re-extracted twice with equal volume of n-butanol. Combined extracts were evaporated to dryness under vacuum at $30^{\circ} \mathrm{C}$. The residue was dissolved in $1 \mathrm{ml}$ of methanol/bi-distilled water (1:9), and the solution was filtered through a $0.2-\mu \mathrm{m}$-Teflon filter. Extracts or and pure culture filtrate were directly subjected to HPLC-mass spectrometry (HPLC-MS) or stored at $-20^{\circ} \mathrm{C}$.

\section{High performance liquid chromatography (HPLC) and mass spectrometric (MS) procedures}

Methanol (gradient quality; Fisher Scientific, Schwerte, Germany), acetonitrile (gradient quality; VWR, Darmstadt, Germany), and acetic acid (p.a.; Fluka) were used for the 
mobile phases.

For nontargeted differential metabolite profiling and $\mathrm{MS}^{\mathrm{n}}$ fragmentation a HPLC system equipped with autosampler (ProStar 410, Varian, Darmstadt, Germany), binary pump system (ProStar 210, Varian), degasser (Degassit, MetaChem Technologies) and a column oven (Jetstream 2 plus, Techlab, Germany) coupled to a ion trap mass spectrometer (500MS, Varian) via electrospray ionization (ESI) source was used.

$10 \mu \mathrm{L}$ of the sample were injected onto a reversed-phase column Polaris C18-Ether $(100 \mathrm{x}$ $2 \mathrm{~mm}$ i.d., $3 \mu \mathrm{m}$ particle size, Varian) with a compatible guard column (MetaGuard Polaris $\mathrm{C} 18$-Ether, Varian) kept at $40{ }^{\circ} \mathrm{C}$. The mobile phase consisted of a binary gradient of $7 \mathrm{mM}$ acetic acid in $95 \%$ water : $5 \%$ acetonitrile (A) and $7 \mathrm{mM}$ acetic acid in methanol (B) $(0-2.20 \min 90 \% \mathrm{~A}: 10 \% \mathrm{~B}, 2.20-25.0 \mathrm{~min}$ from $90 \% \mathrm{~A}: 10 \% \mathrm{~B}$ to $2 \% \mathrm{~A}: 98 \% \mathrm{~B}, 25-$ $30 \min 2 \% \mathrm{~A}: 98 \% \mathrm{~B}$, followed by washing and re-equilibration steps at a flow rate of $0.2 \mathrm{ml} / \mathrm{min})$.

ESI source were operated either with negative or positive spray polarity, the parameters were set as follows (negative/ positive): needle voltage $-3500 \mathrm{~V} /+5000 \mathrm{~V}$, shield voltage $-600 \mathrm{~V} /+600 \mathrm{~V}$, capillary voltage $-/+50 \mathrm{~V}$, drying gas (nitrogen) $20 \mathrm{psi}(138 \mathrm{kPa})$ at $250{ }^{\circ} \mathrm{C}$ and nebulizing gas (-ESI air, +ESI nitrogen) $50 \mathrm{psi}(345 \mathrm{kPa})$. The MS analyzer was operated in full scan mode, mass range m/z 100-1000, scan speed $5000 \mathrm{Da} / \mathrm{sec}$, 3 scans averaged, multiplier voltage set to $1385 \mathrm{~V}$. For fragmentation of candidate signals the ion trap was operated in data-depending-scanning mode using TurboDDS ${ }^{\mathrm{TM}}$ feature (Varian) in this system. For data acquisition MS workstation / MS Data Review 6.9 (Varian) was used. Conversion of raw data format to netCDF was performed with MS Workstation 6.5 .

\section{Selective ion monitoring and product ion scan using triple quadrupole MS}

Verification of canditate signals was performed on a HPLC system consisting of autosampler (ProStar 430, Varian), binary pump system (ProStar 210, Varian), degasser (Degassit, MetaChem Technologies), and column oven (Prostar 510, Varian) connected to photodiode array detector (ProStar 330, Varian) and a triple-quadrupole mass spectrometer (1200 L, Varian) with electrospray interface. 
The chromatographic separation was carried out under the same conditions as described above for nontargeted profiling. The eluate was passed through the cell of a diode array detector (200-800 $\mathrm{nm})$ and subsequently subjected to negative electrospray ionization with the following settings: Needle Voltage $-4400 \mathrm{~V}$, Shield Voltage $-600 \mathrm{~V}$, Capillary voltage $-40 \mathrm{~V}$, Drying gas (nitrogen) $18 \mathrm{psi}(124 \mathrm{kPa})$ at $250{ }^{\circ} \mathrm{C}$, nebulizing gas (air) $50 \mathrm{psi}$ $(345 \mathrm{kPa})$. The candidate ions were detected in selective ion monitoring mode in parallel. When performing product ion scans, the collision energy was set to $20 \mathrm{eV}$ and the collision gas was argon at a pressure of 1.4 mTorr.

MS Workstation 6.5 (Varian) was used for data acquisition, data analysis and integration of peak areas. Peaks simultaneously recorded with diode array detector were used to normalize mass spectrometry signals detected in selective ion mode.

\section{Data processing for nontargeted profiling}

For data processing, XCMS Analyte profiling software Version 1.5.2 running under R package 2.4.0 (Smith et al., 2006) and a user-written Perl scripts applied with ActivePerl 5.8.8 were used. Raw data files were converted into netCDF format. XCMS was used for peak recognition, retention time alignment and peak matching across all samples preclassified into defined groups of samples such as infected and uninfected. XCMS output consisted of a list of aligned peaks defined by mass-to-charge ratio and retention time and their peak areas for all samples of the data set. XCMS generated peak list was used for the normalization of signal intensities performed on the data itself with the help of a trimmedmean algorithm which was originally developed for cDNA differential display data (Venkatesh et al. 2005). The algorithm, implemented in a user-written Perl script, calculates a normalization factor from a subset of signal intensity ratios, excluding signals which are likely to be induced or suppressed by the infection.

Finally comparative analysis of normalized signal intensities was performed by applying a t-test implemented in the Perl script. 


\section{Statistics}

Significance of differences in signal intensities (peak area) among uninfected, $V$. longisporum-infected, and V. dahliae-infected plants was determined by ANOVA, and the significance of correlation coefficients was determined with 1-tailed t tests (SPSS 15.0; SPSS Inc., Chicago, Illinois, USA).

\section{Quantification of $V$. longisporum DNA in hypocotyls and shoots of B. napus}

Fungal biomass was determined in frozen hypocotyls and shoots from single plants harvested from 14-35 dpi. Total DNA was isolated using the cetyltrimethylammonium bromide (CTAB) method as modified by Brandfass and Karlovsky (2006), except for the final precipitation with polyethylene glycol (polyethylene glycol 6000, Serva, Heidelberg, Germany), which was performed as follows. After chloroform extraction, $700 \mu$ of the DNA solution in CTAB buffer was transferred to a $1.5-\mathrm{ml}$ tube containing $200 \mu \mathrm{l}$ polyethylene glycol (30\%) and $100 \mu 15 \mathrm{M} \mathrm{NaCl}$; the solution was mixed, incubated for $20 \mathrm{~min}$ at room temperature, and centrifuged for $15 \mathrm{~min}$ at $14,000 \mathrm{~g}$. The pellet was washed with 70\% (v/v) ethanol, dried, and dissolved in $30 \mu \mathrm{TE}$ buffer $(10 \mathrm{mM}$ Tris/ $\mathrm{HCl}$, 1 mM EDTA, pH 8.0). DNA concentration was estimated by densitometry after electrophoresis in $0.8 \%$ (w/v) agarose gels (Cambrex, Rockland, ME, USA) in TAE buffer (40 mM Tris, $1 \mathrm{mM}$ EDTA, $\mathrm{pH}$ set to 8.5 ) at $7 \mathrm{~V} \mathrm{~cm}^{-1}$ for $60 \mathrm{~min}$. Densitometry was performed as described before (Brandfass and Karlovsky 2006). Fungal DNA was quantified by real-time PCR as reported by Eynck et al. (2007).

\section{ACKNOWLEDGEMENTS}

This work was funded by Deutsche Forschungsgemeinschaft DFG (project FOR546). We thank Varian Deutschland GmbH for excellent technical support and advice. Technical assistance of Monika Boßmann, Patricia Bartoschek, Claudia Nordmann, and Ines Kunze in xylem sap extraction is acknowledged. 


\section{LITERATURE CITED}

Allwood, J.W., Ellis, D.I., Heald, J.K., Goodacre, R., Mur, L.A.J. (2006) Metabolomic approaches reveal that phosphatidic and phosphatidyl glycerol phospholipids are major discriminatory non-polar metabolites in responses by Brachypodium distachyon to challenge by Magnaporthe grisea. Plant J 46(3):351-368

Allwood, J.W., Ellis, D.I., Goodacre, R. (2008) Metabolomic technologies and their application to the study of plants and plant-host interactions. Physiol Plant 132(2):117-135

Alvarez, S., Marsh, E.L., Schroeder, S.G., Schachtman, D.P. (2008) Metabolomic and proteomic changes in the xylem sap of maize under drought. Plant Cell Environ 31(3):325340

Bednarek, P., Schneider, B., Svatos, A., Oldham, N.J., Hahlbrock, K. (2005) Structural complexity, differential response to infection, and tissue specificity of indolic and phenylpropanoid secondary metabolism in Arabidopsis roots. Plant Physiol 138(2):10581070

Birkemeyer, C., Kopka, J. (2007) Design of metabolite recovery by variations of the metabolite profiling protocol. In: Nikolau BJ, Wurtele ES (ed) Concepts in Plant Metabolomics. Springer, Dordrecht, The Netherlands, pp 45-69

Brandfass, C., Karlovsky, P. (2006) Simultaneous detection of Fusarium culmorum and F. graminearum in plant material by duplex PCR with melting curve analysis. BMC Microbiol 6:4

Dunn, W.B., Broadhurst, D., Brown, M., Baker, P.N., Redman, C.W., Kenny, L.C., Kell D.B. (2008) Metabolic profiling of serum using Ultra Performance Liquid Chromatography and the LTQ-Orbitrap mass spectrometry system. J Chromatogr B Analyt Technol Biomed Life Sci doi:10.1016/j.jchromb.2008.03.021 
Eynck, C., Koopmann, B., Grunewaldt-Stoecker, G., Karlovsky, P., von Tiedemann A. (2007) Differential interactions of Verticillium longisporum and V. dahliae with Brassica napus detected with molecular and histological techniques. Eur J Plant Pathol 118:259-274

Fradin, E.F., Thomma, B.P.H.J. (2006) Physiology and molecular aspects of Verticillium wilt diseases caused by V.dahliae and V. albo-atrum. Molecular Plant Pathology 7:71-86

Hagemeier, J., Schneider, B., Oldham, N.J., Hahlbrock, K. (2001) Accumulation of soluble and wall-bound indolic metabolites in Arabidopsis thaliana leaves infected with virulent or avirulent Pseudomonas syringae pathovar tomato strains. Proc Natl Acad Sci U S A 98(2):753-758

Jahangir, M., Kim, H.K., Choi, Y.H., Verpoorte, R. (2008) Metabolomic response of Brassica rapa submitted to pre-harvest bacterial contamination. Food Chemistry 107(1):362-368

Johansson, A., Staal, J., Dixelius, C. (2006) Early responses in the ArabidopsisVerticillium longisporum pathosystem are dependent on NDR1, JA- and ET-associated signals via cytosolic NPR1 and RFO1. Mol Plant Microbe Interact 19:958-969

Karapapa, V.K., Bainbridge, B.W., Heale, J.B. (1997) Morphological and molecular characterisation of Verticillium longisporum comb. nov., pathogenic to oilseed rape. Mycological Research 101: 1281- 1294

Neumann, M.J., Dobinson, K.F. (2003) Sequence tag analysis of gene expression during pathogenic growth and microsclerotia development in the vascular wilt pathogen Verticillium dahliae. Fungal Genet Biol 38(1):54-62

Nordström, A., O'Maille, G., Qin, C., Siuzdak, G. 2006. Nonlinear data alignment for UPLC-MS and HPLC-MS based metabolomics: quantitative analysis of endogenous and exogenous metabolites in human serum. Anal Chem. 78:3289-3295. 
Pedras, M.S., Okanga, F.I., Zaharia, I.L., Khan, A.Q. (2000) Phytoalexins from crucifers: synthesis, biosynthesis, and biotransformation. Phytochemistry 53(2):161-176

Pedras, M.S., Nycholat, C.M., Montaut, S., Xu, Y., Khan, A.Q. (2002) Chemical defenses of crucifers: elicitation and metabolism of phytoalexins and indole-3-acetonitrile in brown mustard and turnip. Phytochemistry 59(6):611-625

Pedras, M.S., Ahiahonu, P.W. (2005) Metabolism and detoxification of phytoalexins and analogs by phytopathogenic fungi. Phytochemistry 66(4):391-411

Pedras, M.S., Adio, A.M., Suchy, M., Okinyo, D.P., Zheng, Q.A., Jha, M., Sarwar, M.G. (2006) Detection, characterization and identification of crucifer phytoalexins using highperformance liquid chromatography with diode array detection and electrospray ionization mass spectrometry. J Chromatogr A 1133(1-2):172-183

Pedras, M. S., Jha, M., Minicm, Z., Okeola, O. G. (2007) Isosteric probes provide structural requirements essential for detoxification of the phytoalexin brassinin by the fungal pathogen Leptosphaeria maculans. Bioorg Med Chem. 15:6054-6061.

Pedras, M S, Zheng, Q-A, Gadagi, R S, Rimmer, S R (2008) Phytoalexins and polar metabolites from the oilseeds canola and rapeseed: Differential metabolic responses to the biotroph Albugo candida and to abiotic stress. Phytochemistry 69: 894-910

Smith, C.A., Want, E.J., O'Maille, G., Abagyan, R., Siuzdak, G. (2006) XCMS: processing mass spectrometry data for metabolite profiling using nonlinear peak alignment, matching, and identification. Anal Chem 78(3):779-787

Wikoff, W.R., Gangoiti, J.A., Barshop, B.A., Siuzdak, G. (2007) Metabolomics identifies perturbations in human disorders of propionate metabolism. Clin Chem 53(12):2169-2176

Venkatesh, B., Hettwer, U., Koopmann, B., Karlovsky, P. 2005. Conversion of cDNA differential display results (DDRT-PCR) into quantitative transcription profiles. BMC Genomics. 6:51 DOI 10.1186/1471-2164-6-51 
Zeise, K., von Tiedemann, A. (2001) Morphological and physiological differentiation among vegetative compatibility groups of Verticillium dahliae in relation to Verticillium longisporum. J Phytopathology 149:469-475

Zhou, L., Hu, Q., Johansson, A., Dixelius, C. (2006) Verticillium longisporum and Verticillium dahliae: infection and disease in Brassica napus. Plant Pathol 55:137-144

\section{Optional Author-Recommended Internet Resources}

KEGG database http://www.genome.jp/kegg/ligand.html 


\section{TABLES AND FIGURES}

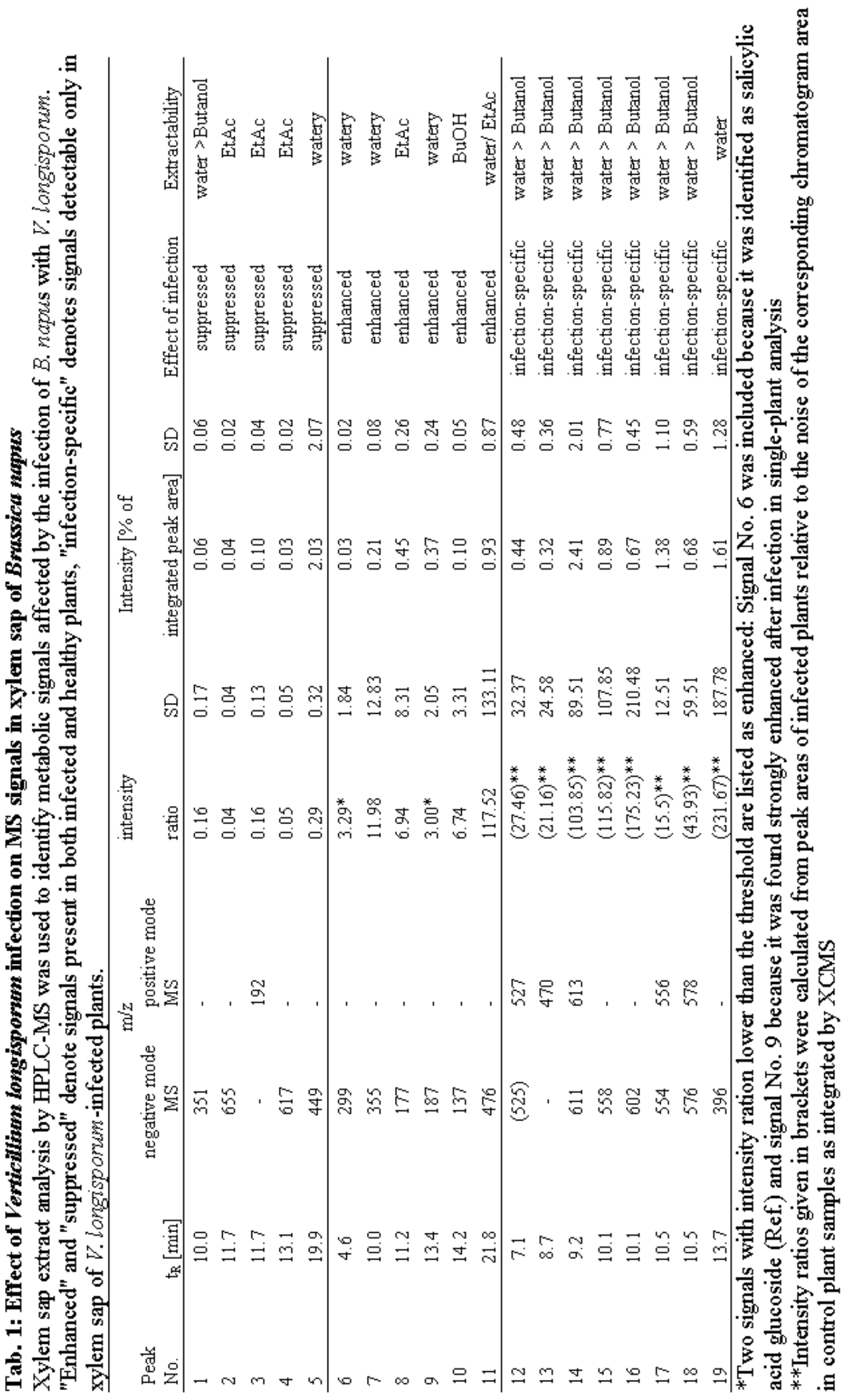


Tab. 2: Correlations metabolite versus metabolite (peak areas)

Pairwise correlation ( $\mathrm{x}$ versus $\mathrm{y}$ ) of signal intensities of infection-specific metabolites identified by $m / z$ value. Pearson correlation is given as (r), significance at $p \leq 0.05$ (1-tailed $t$ test) is given as (s), not significant (ns) at $\mathrm{p}>0.05$. Xylem sap samples (n) pooled from 4 plants are designated as pools.

\begin{tabular}{|c|c|c|c|c|}
\hline $\mathrm{x}(\mathrm{m} / \mathrm{z})$ & $\mathrm{y}(\mathrm{m} / \mathrm{z})$ & $\mathrm{r}$ & $\mathrm{n}$ (pools) & significance \\
\hline 558 & 554 & 0.896 & 20 & $\mathrm{~s}$ \\
\hline 396 & 554 & 0.951 & 20 & $\mathrm{~s}$ \\
\hline 396 & 611 & 0.895 & 20 & $\mathrm{~s}$ \\
\hline 558 & 611 & 0.842 & 20 & $\mathrm{~s}$ \\
\hline 611 & 554 & 0.902 & 20 & $\mathrm{~s}$ \\
\hline 396 & 558 & 0.966 & 20 & $\mathrm{~s}$ \\
\hline 558 & 476 & 0.927 & 20 & $\mathrm{~s}$ \\
\hline 396 & 476 & 0.886 & 20 & $\mathrm{~s}$ \\
\hline 611 & 476 & 0.771 & 20 & $\mathrm{~s}$ \\
\hline 554 & 476 & 0.809 & 20 & $\mathrm{~S}$ \\
\hline 612 & 555 & 0.900 & 16 & $\mathrm{~s}$ \\
\hline 527 & 470 & 0.824 & 16 & $\mathrm{~s}$ \\
\hline 612 & 527 & 0.856 & 16 & $\mathrm{~S}$ \\
\hline 612 & 470 & 0.765 & 16 & $\mathrm{~s}$ \\
\hline 612 & 470 & 0.837 & 13 & $\mathrm{~S}$ \\
\hline $\mathrm{x}(\mathrm{m} / \mathrm{z})$ & $\mathrm{y}(\mathrm{m} / \mathrm{z})$ & $\mathrm{r}$ & $\begin{array}{l}\mathrm{n} \text { (individual } \\
\text { plants) }\end{array}$ & significance \\
\hline 558 & 396 & 0.901 & 6 & $\mathrm{~s}$ \\
\hline 611 & 554 & 0.987 & 6 & $\mathrm{~S}$ \\
\hline 611 & 558 & 0.193 & 6 & ns \\
\hline 611 & 396 & 0.155 & 6 & ns \\
\hline 554 & 558 & 0.094 & 6 & ns \\
\hline 554 & 396 & 0.079 & 6 & ns \\
\hline
\end{tabular}

Tab. 3: Putative building blocks composing infection-specific signals

\begin{tabular}{ccccl} 
No. & $\begin{array}{c}\mathrm{t}_{\mathrm{R}} \\
{[\mathrm{min}]}\end{array}$ & $\begin{array}{c}\text { signal } \\
(\mathrm{m} / \mathrm{z})\end{array}$ & {$[\mathrm{M}]$} & Putative building blocks \\
\hline 1 & 8.7 & 470 & 469 & ß-D-Glucopyranosyl indole-3-carboxylic acid, block ${ }^{1}$ \\
2 & 7.1 & 527 & 526 & $\begin{array}{l}\text { Indole-3 -pyruvate, ß-D-glucopyranosyl indole-3- } \\
\text { carboxylic acid }\end{array}$ \\
3 & 10.5 & $554 / 556$ & 555 & $\begin{array}{l}\mathrm{CO}_{2} \text {, tryptophan, indole-3-carboxylic acid, block } \\
1\end{array}$ \\
4 & 9.2 & $611 / 613$ & 612 & $\begin{array}{l}\text { Indole-3-pyruvate, } \mathrm{CO}_{2} \text {, tryptophan, indole-3- } \\
\text { carboxylic acid } \\
5\end{array}$ \\
13.7 & 396 & 397 & $\begin{array}{l}\text { Tryptophan, 5,6-dihydroxyindole-2-carboxylate } \\
\text { Tryptophan, indole-3-acetic acid, glucose }\end{array}$ \\
6 & 10.1 & 558 & 559 &
\end{tabular}




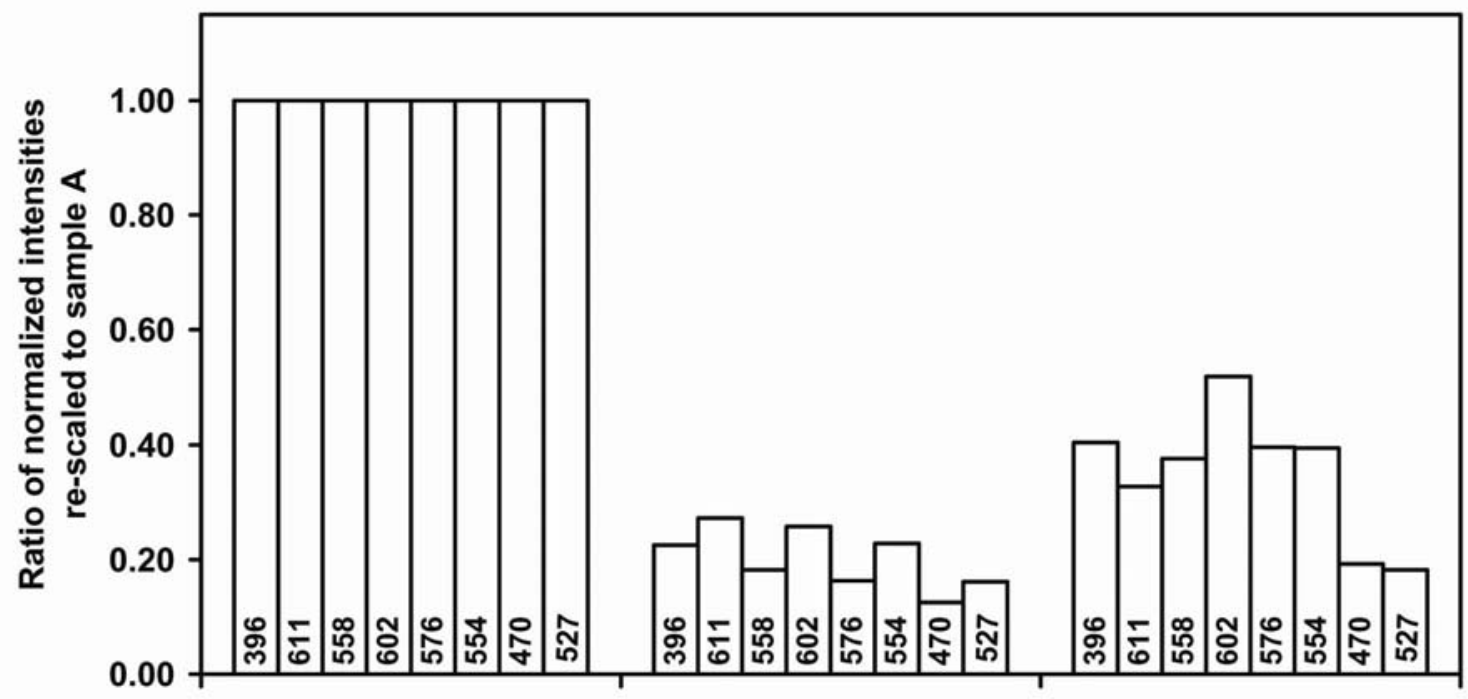

A

B

C

Xylem sap pools

Fig. 1: Intensity of infection-specific signals and disease symptoms

Each xylem sap sample (A, B, C) was pooled from four $V$. longisporum-diseased plants sorted by the stunting symptom (plant height) into three groups. A: plant height $<30 \mathrm{~cm}$ (all plants in this group were smaller than $20 \mathrm{~cm}$ ). B: plant height $30-35 \mathrm{~cm}$. C: plant height $35-40 \mathrm{~cm}$.

Signals $\mathrm{m} / \mathrm{z} 470$ and 527 were detected in positive ionization mode, the remaining signals in negative mode. 

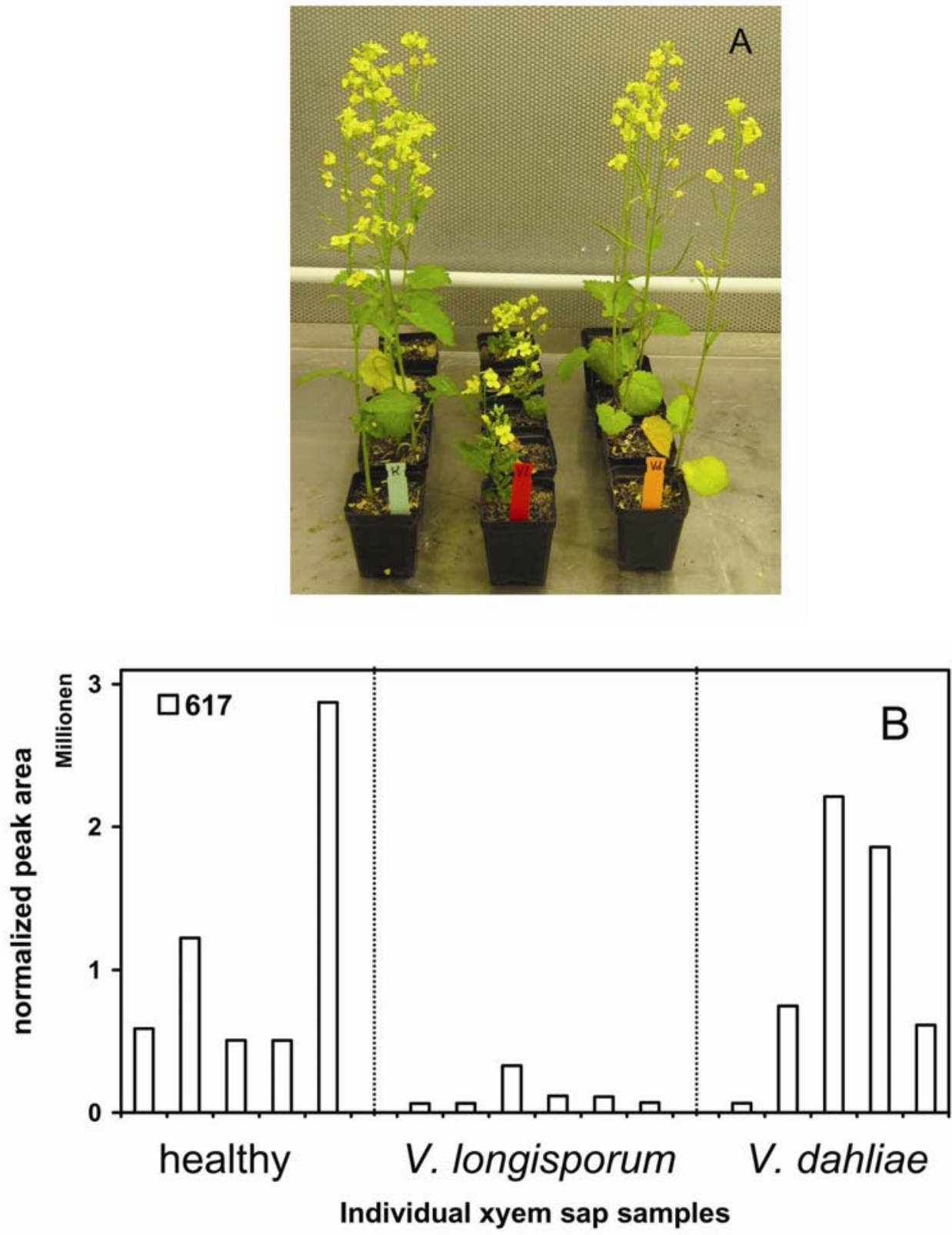

Fig. 2: Analysis of individual plants

A: Individual rapid cycling rape plants 28 days after infection with $V$. longisporum (middle) or $V$. dahliae (right). Control plants were mock-inoculated with water (left). B: Intensity of a signal $\mathrm{m} / \mathrm{z} 617$ in xylem sap from single $B$. napus plants infected with $V$. longisporum (middle, $\mathrm{n}=6$ ) V. dahliae (right, $\mathrm{n}=5$ ) and healthy control (left, $\mathrm{n}=5$ ) at 28 dpi. Signal intensity is diminished in plants infected with $V$. longisporum (middle) but not in V. dahliae-infected (right) and healthy plants (left). 

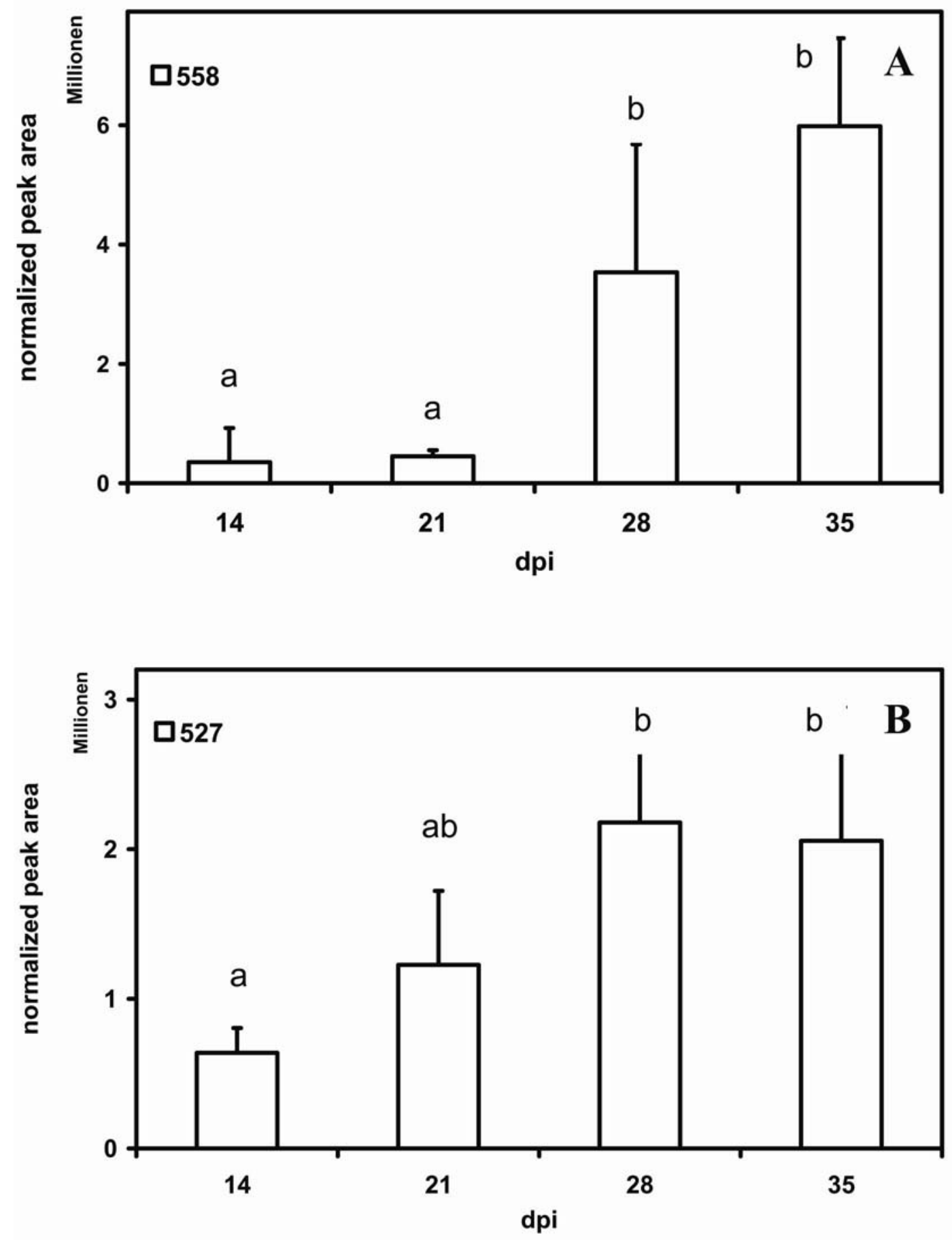

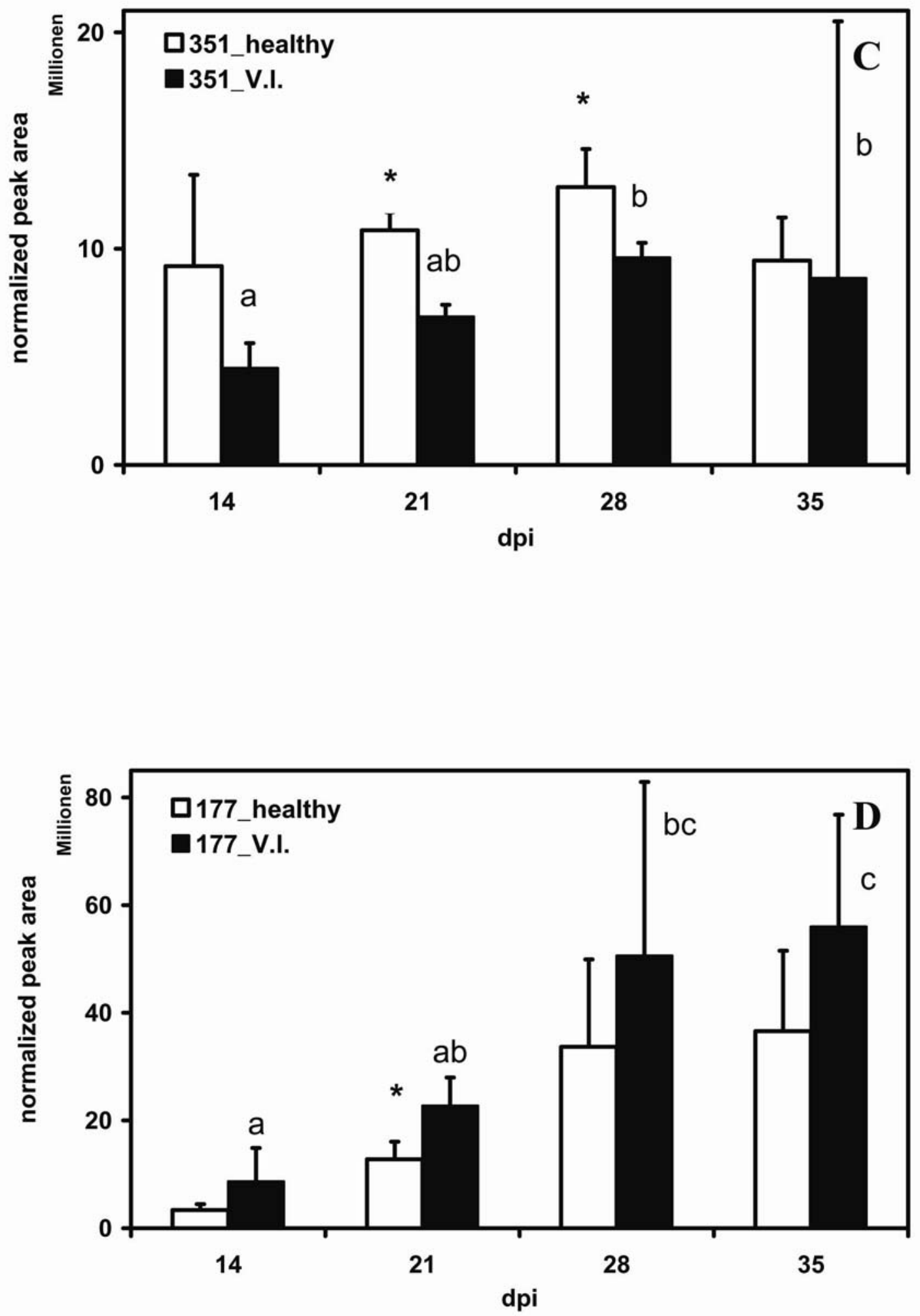

Fig. 3: Development of signal intensities in time

A-D: Intensities of selected metabolic signals in xylem sap from $V$. longisporum-infected $B$. napus plants and non-infected healthy plants during disease development (14-35 dpi). Each bar depicts an average of 5 xylem sap samples, each pooled from 4 plants. Standard deviation is shown as error bars. Statistical significance of differences among time points is 
indicated for $\mathrm{p}<0.05$ (Tukey). Values showing significant differences $(\mathrm{P} \leq 0.05)$ between xylem sap samples from $V$. longisporum-infected plants and samples from healthy plants are labeled by asterisks (one tailed t-test). Signal 558 (A) and 527 (B) only occured in $V$. longisporum-infected plants.

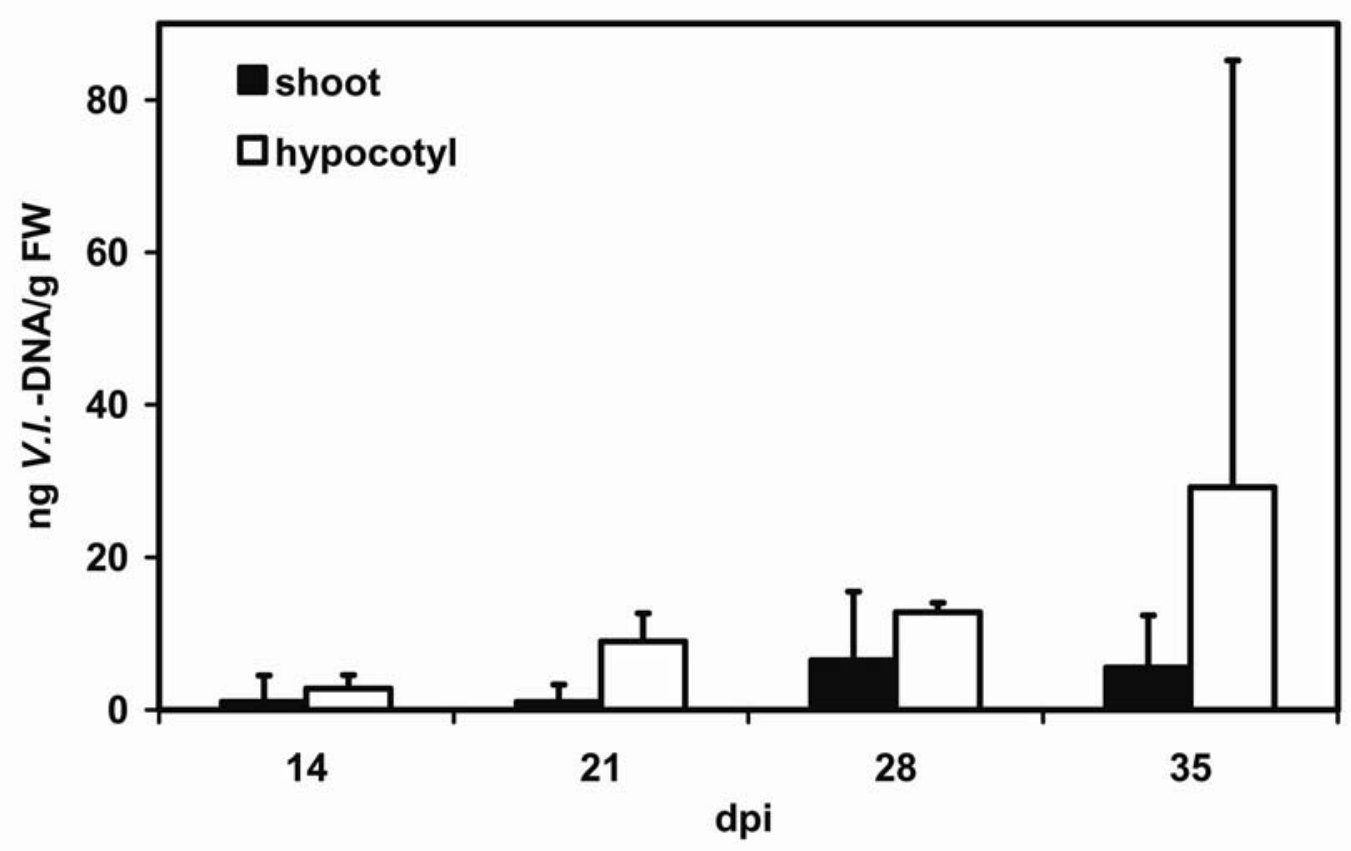

Fig. 4: Development of fungal DNA amount in plant over time

Amount of $V$. longisporum-DNA (ng DNA/gFW) detected by real-time PCR in hypocotyl (white bars) and shoot tissue (black bars) of infected plants during disease development (14-35dpi). $n=10$. 


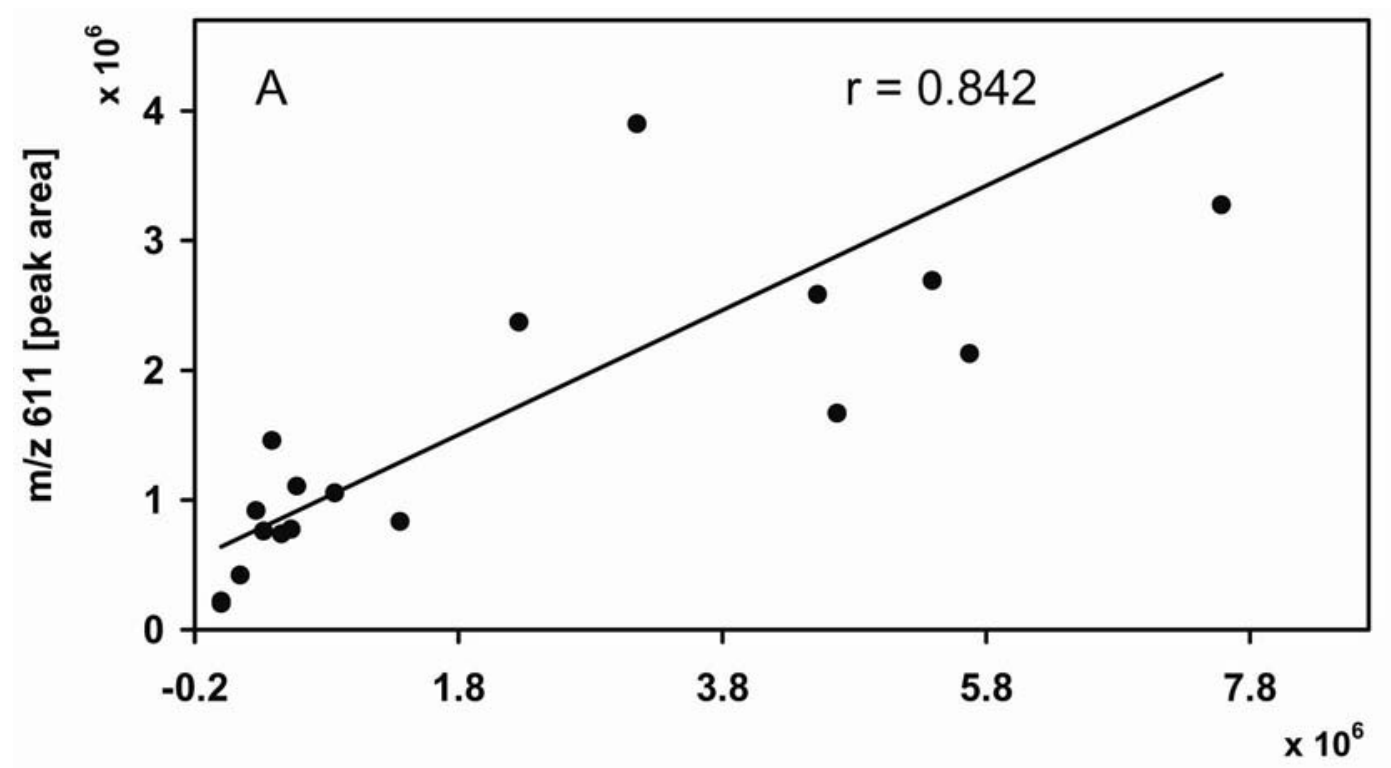

$\mathrm{m} / \mathrm{z} 558$ [peak area]

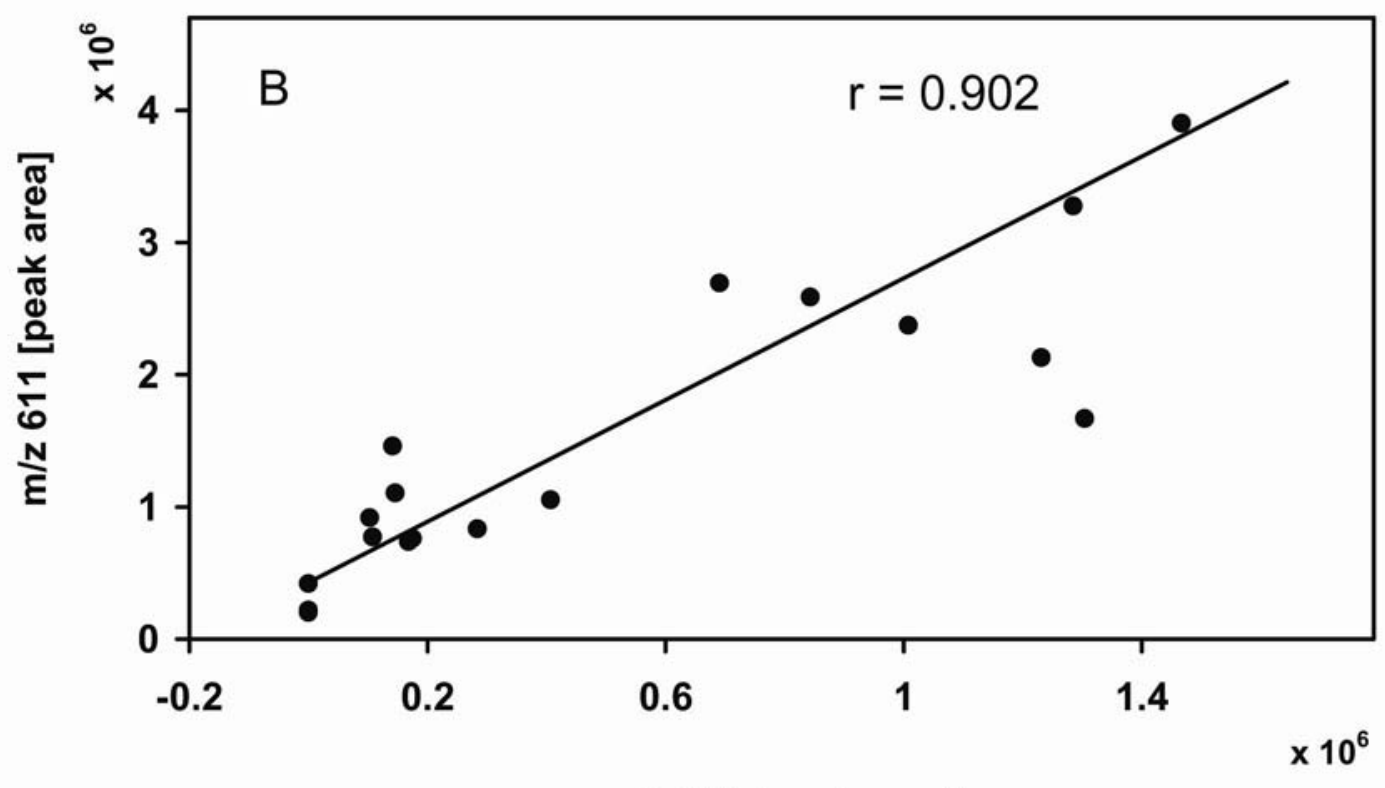

m/z 554 [peak area] 


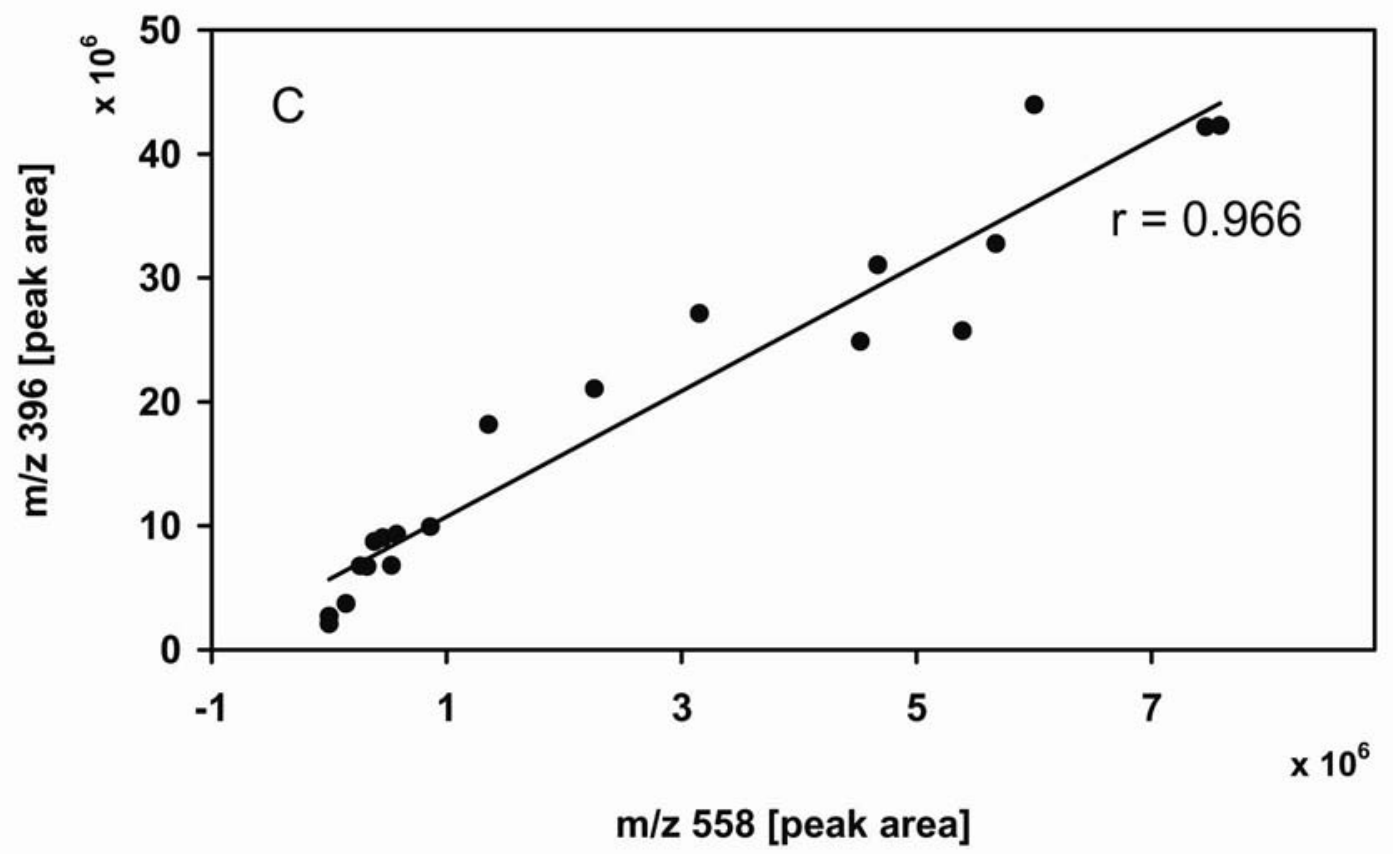

Fig. 5: Metabolite-metabolite-correlations among xylem sap pools of infected plants Correlation of signal intensities: A) $\mathrm{m} / \mathrm{z} 558$ (x-axis) versus $\mathrm{m} / \mathrm{z} 611$ (y axis); B) $\mathrm{m} / \mathrm{z} 554$ (x-axis) versus $m / z 611$ (y axis); C) m/z 558 (x-axis) versus $m / z 396$ (y axis).

Each point $(\bullet)$ depicts a xylem sap sample pooled from 4 plants. Pearson correlation is given as ( $\mathrm{r}$ ), all correlations are significant at $\mathrm{P} \leq 0.05$ (1-tailed $\mathrm{t}$ test). 
1 $m / z 470[\mathrm{M}+\mathrm{H}]^{+} \quad m / z 527[\mathrm{M}+\mathrm{H}]^{+}$
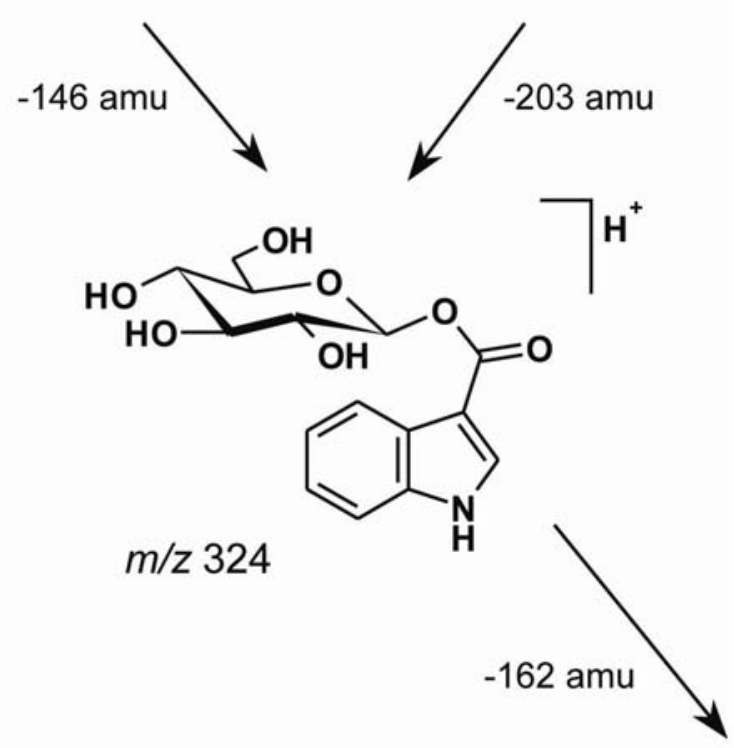

3 $m / z 556[\mathrm{M}+\mathrm{H}]^{+} \quad m / z 613[\mathrm{M}+\mathrm{H}]^{+}$
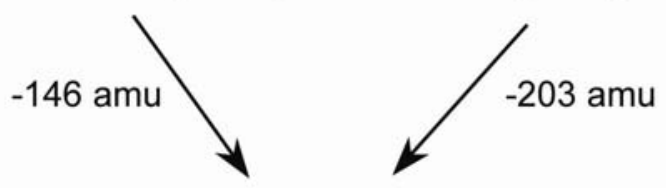

$m / z 410$<smiles>C[C+]CC</smiles>

$m / z 162$<smiles>C[C+]1CCCCC1</smiles>

$m / z 118$

Fig. 6: Fragmentation scheme of infection-specific signals in positive ESI

Fragmentation scheme of infection specific signals in positive ESI mode based on results from multiple stage data-dependent fragmentation on an ion trap MS. 


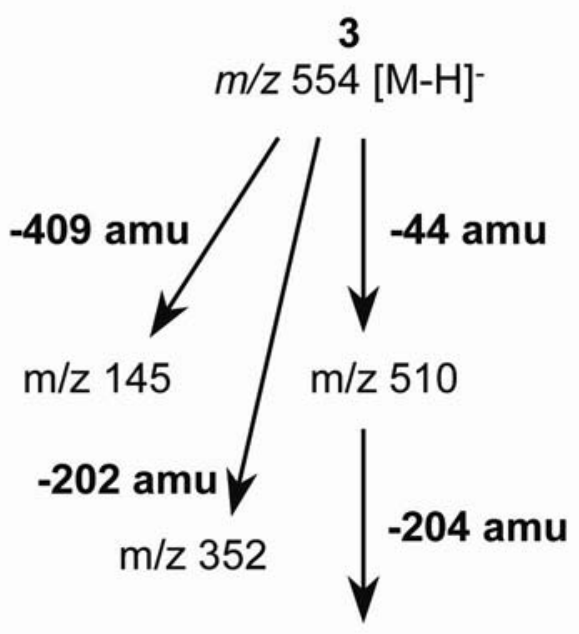

$\mathrm{m} / \mathrm{z} 306$

5

$\mathrm{m} / \mathrm{z} 396$ [M-H] $^{-}$

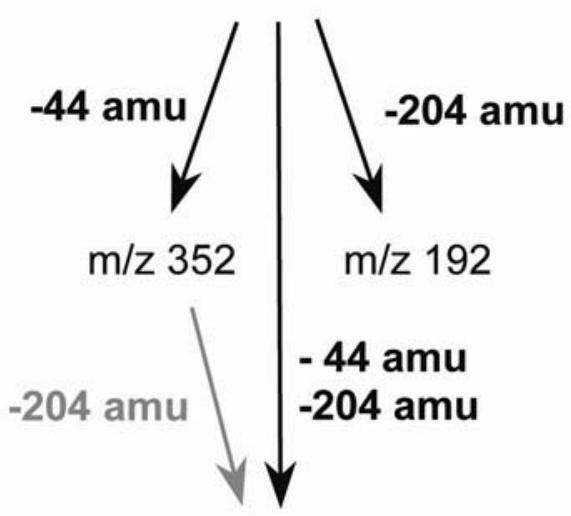

$m / z 148$
4

$\mathrm{m} / \mathrm{z} 611[\mathrm{M}-\mathrm{H}]^{-}$

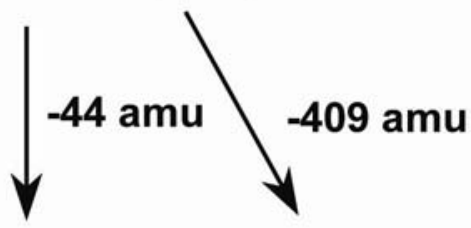

$\mathrm{m} / \mathrm{z} 567 \quad \mathrm{~m} / \mathrm{z} 202$

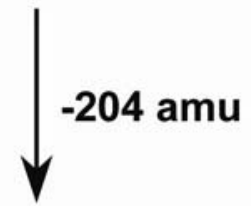

$\mathrm{m} / \mathrm{z} 363$

6

$m / z 558$ [M-H] $^{-}$

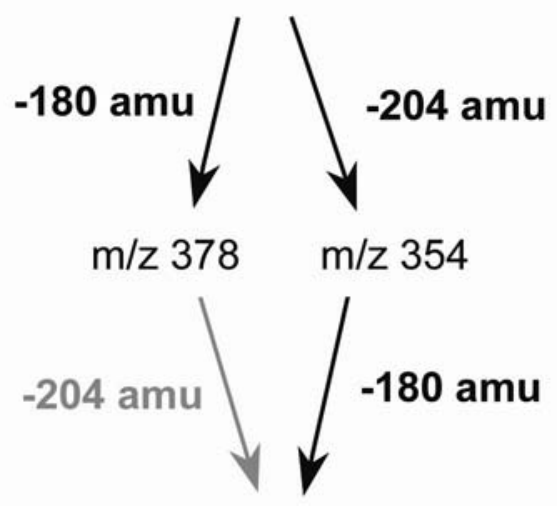

$m / z 174$

Fig. 7: Fragmentation scheme of infection-specific signals in negative ESI

Fragmentation scheme of infection specific signals in negative ESI mode based on results from product ion scans on a triple quadrupole MS and multiple stage data-dependent fragmentation on an ion trap MS. Grey arrows represent a minor fragmentation. 


\title{
Chapter 3: Identification of salicylic acid glucoside in xylem sap of Brassica napus and the effect of infection with Verticillium longisporum*
}

*This manuscript has been submitted for publication.

Astrid Ratzinger ${ }^{1}$, Nadine Riediger ${ }^{2}$, Andreas von Tiedemann ${ }^{2}$, Petr Karlovsky $^{1 *}$

${ }^{1}$ Molecular Phytopathology and Mycotoxin Research Unit \& ${ }^{2}$ Plant Pathology and Plant Protection Unit, Department of Crop Sciences, Goettingen University, Grisebachstrasse 6, 37077 Goettingen, Germany

\begin{abstract}
Salicylic acid (SA) and its glucoside (SAG) were detected by HPLC-MS in xylem sap of uninfected Brassica napus. Concentrations of SA and SAG both in xylem sap and in shoot extracts increased after infection of the plant with the vascular pathogen Verticillium longisporum. The elevation of SA and SAG were correlated with disease severity assessed as the reduction of shoot length. Similarly, SAG levels in shoot extracts were strongly correlated with the amount of fungal DNA in the hypocotyls of single plants infected with $V$. longisporum. At 14 days post inoculation (dpi) and relative to uninfected controls, the xylem sap of plants infected with $V$. longisporum contained about three times more SA and about five times more SAG. Salicylic acid glucoside (but not SA) levels in infected plants gradually declined with time until 35 dpi, even though they remained higher in infected than in uninfected plants. Jasmonic acid (JA) and abscisic acid (ABA) levels in xylem sap were not affected by infection of B. napus with $V$. longisporum. Levels of SA, SAG, JA, and ABA were unaffected by infection with $V$. dahliae. These data indicate that SA and SAG are involved in the response of $B$. napus to infection with $V$. longisporum and may act as long-distance signals transported in the plant xylem.
\end{abstract}


Keywords: Brassica napus, metabolic profiling, salicylic acid, salicylic acid glucoside, Verticillium longisporum, xylem

\author{
Abbreviations \\ ABA cis-Abscisic acid, \\ dpi Days post inoculation, \\ JA Jasmonic acid, \\ $\mathrm{m} / \mathrm{z} \quad$ Mass-to-charge ratio, \\ SA Salicylic acid, \\ SAG Salicylic acid glucoside
}

\title{
Introduction
}

In plants, salicylic acid (SA) is a crucial component of the signal transduction pathway that is triggered by infection and leads to the activation of a complex defense response designated systemic acquired resistance (SAR; Durrant and Dong 2004). Salicylic acid is an essential component of the pathway because the expression of salicylic acid hydroxylase (product of NahG gene) in several plant species abolished SAR (Gaffney et al. 1993). Several studies demonstrated elevated endogenous SA levels in phloem sap upon pathogen attack (Métraux et al. 1990; Yalpani et al. 1991). However, SA does not serve as a mobile signal transmitted from the infected tissue into distal parts of the plant to activate SAR (Rasmussen et al. 1991; Vernooij et al. 1994). Thus, the nature of this signal remains unknown. Investigations on Arabidopsis mutants dir1-1 and sfd1 indicated that the signal is a lipid-derived molecule (Maldonado et al. 2002; Nandi et al. 2004). Truman et al. (2007) recently suggested that the signal of SAR is jasmonic acid, while Park et al. (2007) proposed that it is methyl salicylate (MeSA) alone or MeSA and a lipid-based molecule (Park et al. 2007).

Salicylic acid and its derivatives are transported within plants by different mechanisms. Methyl salicylate is volatile and acts as an airborne mobile signal between TMV-infected leaves and healthy tissue of the same tobacco plant as well as neighboring plants (Shulaev et al. 1997). Transmission of MeSA via vascular tissue was suggested (Shulaev et al. 1997) but not addressed experimentally. The undissociated form of SA may cross phospholipid 
membranes by diffusion and get trapped there in an ionized form (Yazaki et al. 2007). Salicylic acid and its derivatives may also be actively transported between cell compartments, as has been shown for the transport of salicylic acid glucoside (SAG) into the vacuole (Dean and Mills 2004).

Long-distance transport of non-volatile SA derivatives may only occur via the phloem or with the respiration stream in xylem. SA transport via phloem was first suggested based on in vivo-labeling with ${ }^{18} \mathrm{O}_{2}$. Although $\left[{ }^{18} \mathrm{O}\right] \mathrm{SA}$ was produced in TMV-inoculated lower tobacco leaves, $70 \%$ of $\left[{ }^{18} \mathrm{O}\right] \mathrm{SA}$ was detected in uninoculated younger leaf tissue above (Shulaev et al.1995). Another study investigated the distribution of ${ }^{14} \mathrm{C}$-labeled $\mathrm{SA}$ in phloem and upper uninoculated cucumber leaves after injection of ${ }^{14} \mathrm{C}$-labeled benzoic acid accompanied by an inoculation of cotyledons with TNV (Mölders et al. 1996); their results did not support the hypothesis that SAR was induced by phloem-transported SA. Further investigation revealed that the phloem distribution of $\left[{ }^{14} \mathrm{C}\right] \mathrm{SA}$ from the infiltrated leaf within the Arabidopsis rosette could not be explained by phloem allocation alone (Kiefer and Slusarenko 2003). In line with these results, Rocher at al. (2006) hypothesized that SA transport within the plants was not limited to phloem. Exogenously applied $\left[{ }^{14} \mathrm{C}\right]$-labeled SA was transported from cotyledons via the phloem towards the basal part of the seedling, where a small part of SA was exported into the xylem. The authors suggested that, in addition to phloem allocation of SA and endogenous synthesis induced by some unknown signal, long-distance transport of SA via xylem may contribute to SA accumulation in distal parts of the plant in response to pathogen infection of a lower part. They attempted to measure endogenous SA in xylem sap but the concentration was below the limit of detection of HPLC with UV detection.

Verticillium longisporum, a soil-borne fungal pathogen with host-specificity for the genus Brassica, enters host plants through the root and colonizes xylem vessels. Because the pathogen remains restricted to the roots and hypocotyl for extended periods of its infection cycle while the shoots develop disease symptoms (Eynck et al. 2007; Zhou et al. 2006), we hypothesize that a chemical signal(s) transported upward triggers the development of disease symptoms. The most plausible mechanism of signal transport from the root to the shoot is by xylem sap.

In this work we demonstrate that SA and its glucoside are present in xylem sap of healthy Brassica napus and investigate how their levels are affected by infection with Verticillium longisporum. 


\section{Material and Methods}

\section{Plant material}

Rapid-cycling rape, (Brassica napus var. napus, genome ACaacc) was provided by Paul H. Williams (Department of Plant Biology, University of Wisconsin-Madison, Crucifer Genetics Cooperative, Stock number 5). Seeds were surface sterilized by immersing them into $70 \%$ ethanol for $30 \mathrm{~s}$ and then were rinsed with sterilized tap water for $30 \mathrm{~s}$ three times.

\section{Fungal isolates}

Verticillium longisporum VL 43 from Brassica napus and Verticillium dahliae VD 73 from Linum usitatissimum were used in this study. Both strains were isolated from plants grown in northern Germany as described in Zeise and von Tiedemann (2001).

Fungal stocks with $1-3 \times 10^{6}$ conidia per $\mathrm{ml}$ were stored at $-80^{\circ} \mathrm{C}$ in $25 \%$ glycerol.

\section{Preparation of inoculum for plant infection}

Shake cultures were started by adding $500 \mu \mathrm{L}$ of the spore suspension $\left(1-3 \times 10^{6}\right.$ conidia

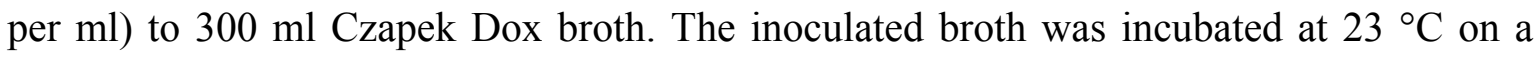
rotary shaker at $100 \mathrm{rpm}$ in the dark. After 10-14 days, the culture was filtered through a sterile gauze. Spore concentration was determined using a haemocytometer and was diluted to $1 \times 10^{6}$ spores per $\mathrm{ml}$.

\section{Plant inoculation and cultivation}

Rapid-cycling rapeseeds were germinated in autoclaved sand in climate-controlled chambers $\left(30.0 \mathrm{kLux}, 60 \%\right.$ humidity, $22 / 20{ }^{\circ} \mathrm{C}$ day/night, and 15 -h day length; Philips TL5 HO lamps). Seven-day-old seedlings were inoculated by root-dipping in a spore suspension of either $V$. longisporum (isolate 43, $1 \times 10^{6}$ spores per $\mathrm{ml}$ ) or $V$. dahliae (isolate $73,1 \mathrm{x}$ $10^{6}$ spores per $\mathrm{ml}$ ) for $30-45$ minutes. As a control, seedlings were dipped in sterile water. After inoculation, single seedlings were potted in a sterile sand-soil mixture (1:1) and grown under the conditions described above. 


\section{Xylem sap preparation}

Rapeseed plants were harvested 28 days post inoculation (dpi). For time-course experiments, xylem sap was collected 14, 21, 28, and 35 dpi. Shoots were cut above the hypocotyl, and xylem sap was collected by pressurizing roots to 4 x $10^{5} \mathrm{~Pa}$ for $15 \mathrm{~min}$ using a Scholander pressure chamber (Scholander et al. 1965). Xylem sap was filtered through a $0.2-\mu \mathrm{m}$-Teflon filter (WICOM, Heppenheim, Germany) and subjected to HPLCMS analysis immediately or stored at $-20{ }^{\circ} \mathrm{C}$. Xylem sap was checked for contamination with cytoplasm by determining malate dehydrogenase activity (absence of activity indicated absence of cytoplasm contamination).

\section{Preparation of shoot extracts}

The shoots of plants from which xylem sap was obtained were shock frozen with liquid nitrogen and stored at $-80{ }^{\circ} \mathrm{C}$. Frozen shoot material was lyophilized (at $-20{ }^{\circ} \mathrm{C}$ ) for $24 \mathrm{~h}$. Dry shoot material was finely ground in a ball mill. Extraction protocol described by Birkemeyer and Kopka (2007) was modified as follows. To $50 \mathrm{mg}$ of dry shoot material (two replications for each plant), $1.5 \mathrm{ml}$ methanol (gradient quality; Fisher Scientific, Schwerte, Germany) and $1.0 \mathrm{ml}$ chloroform (p.a.; Roth, Karlsruhe, Germany) were added. The mixture was vigorously shaken and incubated for $5 \mathrm{~min}$ at $37{ }^{\circ} \mathrm{C}$. Subsequently, $2 \mathrm{ml}$ of either twice-distilled water or $2 \%$ formic acid (puriss. p.a.; Fluka, Seelze, Germany) in water were added. The suspension was shaken for $2 \mathrm{~h}$ at room temperature. The samples were centrifuged at $3583 \mathrm{~g}$ for $15 \mathrm{~min}$, the phases were separated, and each phase was evaporated under vacuum to dryness at $30{ }^{\circ} \mathrm{C}$. The residue of the polar phase was dissolved in $1 \mathrm{ml}$ of methanol/bi-distilled water (1:9), and the solution was filtered through a $0.2-\mu \mathrm{m}$ Teflon filter. Samples were directly subjected to HPLC-mass spectrometry (HPLC-MS) or stored at $-20{ }^{\circ} \mathrm{C}$.

\section{High performance liquid chromatography and mass spectrometry}

Methanol (gradient quality; Fisher Scientific, Schwerte, Germany), acetonitrile (gradient quality; VWR, Darmstadt, Germany), and acetic acid (p.a.; Fluka) were used for the mobile phases.

For full-scan analysis, an HPLC system equipped with autosampler (ProStar 410, Varian, 
Darmstadt, Germany), binary pump system (ProStar 210, Varian), degasser, and a column oven coupled to an ion trap mass spectrometer (500-MS, Varian) via electrospray ionization source was used. Samples were injected onto a reversed-phase column Polaris C18-Ether $\left(100 \times 2 \mathrm{~mm}, 3 \mu \mathrm{m}\right.$ particle size; Varian) kept at $40{ }^{\circ} \mathrm{C}$. The mobile phase consisted of a binary gradient of $7 \mathrm{mM}$ acetic acid in $95 \%$ water with $5 \%$ acetonitrile (A) and $7 \mathrm{mM}$ acetic acid in methanol (B): $0.0-2.2 \mathrm{~min} 10 \% \mathrm{~B}, 2.2-25.0 \mathrm{~min}$ from $10 \% \mathrm{~B}$ to $98 \%$ B , 25-30 min 98\% B, followed by washing (98\% B) and re-equilibration (10\% B) steps at a flow rate of $0.2 \mathrm{ml} / \mathrm{min}$. The electrospray ionization source was operated either with negative or positive spray polarity with the mass spectrometer operating in a full scan mode at a mass range of a mass-to-charge ratio $(\mathrm{m} / \mathrm{z}) \quad 100-1000$ and a scan speed of 5000 $\mathrm{Da} / \mathrm{s}$. Raw data files were converted into NetCDF format and processed using XCMS Analyte Profiling Software (Smith et al. 2007).

Phytohormones, SA, and SAG were quantified using multiple reaction monitoring mode on the HPLC system specified above connected to a triple-quadrupole mass spectrometer (1200 L, Varian) with an electrospray interface. Standards of SA, JA, and ABA were purchased from Sigma-Aldrich. Salicylic acid glucoside was kindly provided by Prof. H. Kneifel (Research Centre Jülich GmbH, Institute Phytosphere, Jülich, Germany). The analytes were separated on a reversed-phase column (Polaris C18-A, 125 x $2 \mathrm{~mm}, 5 \mu \mathrm{m}$ particle size, Varian) at $40{ }^{\circ} \mathrm{C}$ and eluted isocratically with $7 \mathrm{mM}$ acetic acid in $47.5 \%$ water, $2.5 \%$ acetonitrile, and $50 \%$ methanol at a flow rate of $0.2 \mathrm{ml} / \mathrm{min}$. The eluate was subjected to negative electrospray ionization, and the ions were detected using the following mass transitions: SAG $\mathrm{m} / \mathrm{z} 298.8 \rightarrow \mathrm{m} / \mathrm{z} 137$ and $\mathrm{m} / \mathrm{z} 298.8 \rightarrow \mathrm{m} / \mathrm{z}$ 93; SA $\mathrm{m} / \mathrm{z}$ $136.8 \rightarrow \mathrm{m} / \mathrm{z}$ 93; ABA $\mathrm{m} / \mathrm{z} 262.8 \rightarrow \mathrm{m} / \mathrm{z}$ 153, JA m/z $208.9 \rightarrow \mathrm{m} / \mathrm{z} 59$.

\section{Statistics}

Data are presented as means \pm standard deviation. The standard deviations for the increases in SA or SAG relative to the control were calculated according to the error propagation formula. Differences in concentrations of phytohormones and salicylic acid derivatives among uninfected, $V$. longisporum-infected, and $V$. dahliae-infected plants were determined by ANOVA, and the significance of correlation coefficients was determined with 1-tailed t-tests (SPSS 15.0; SPSS Inc., Chicago, Illinois, USA). 


\section{Quantification of $V$. longisporum DNA}

Fungal biomass was determined in lyophilized hypocotyls from single plants harvested at 28 dpi. DNA was isolated using the cetyltrimethylammonium bromide (CTAB) method as modified by Brandfass and Karlovsky (2006), except for the final precipitation with polyethylene glycol (polyethylene glycol 6000, Serva, Heidelberg, Germany), which was performed as follows. After chloroform extraction, $700 \mu \mathrm{l}$ of the DNA solution in CTAB buffer was transferred to a $1.5-\mathrm{ml}$ tube containing $200 \mu$ polyethylene glycol $(30 \%)$ and $100 \mu 15 \mathrm{M} \mathrm{NaCl}$; the solution was mixed, incubated for $20 \mathrm{~min}$ at room temperature, and centrifuged for $15 \mathrm{~min}$ at $14,000 \mathrm{~g}$. The pellet was washed with $70 \%(\mathrm{v} / \mathrm{v})$ ethanol, dried, and dissolved in $30 \mu \mathrm{TE}$ buffer $(10 \mathrm{mM}$ Tris/HCl, $1 \mathrm{mM}$ EDTA, pH 8.0). DNA concentration was estimated by densitometry after electrophoresis in $0.8 \%(\mathrm{w} / \mathrm{v})$ agarose gels (Cambrex, Rockland, ME, USA) in TAE buffer (40 mM Tris, $1 \mathrm{mM}$ EDTA, $\mathrm{pH}$ set to 8.5 ) at $7 \mathrm{~V} \mathrm{~cm}^{-1}$ for $60 \mathrm{~min}$. Densitometry was performed as described before (Brandfass and Karlovsky 2006). Fungal DNA was quantified by real-time PCR as described by Eynck et al. (2007).

\section{Results}

\section{Salicylic acid glucoside (SAG) in xylem sap and shoots of Brassica napus}

Xylem sap extracted from Brassica napus plants infected with Verticillium longisporum and from healthy controls was analyzed by HPLC-MS to identify metabolites affected by the infection. Plants at 28 dpi were used because at this time the amount of xylem sap extractable from a single plant exceeded $100 \mu$. Xylem sap was filtered and directly subjected to HPLC-MS. Comparative analysis of metabolic profiles of infected and healthy plants revealed a number of signals with increased intensities in infected plants. One of these signals had a mass-to-charge ratio $(\mathrm{m} / \mathrm{z})$ of 299 , which is the same as molecular ion of SAG in negative ionization mode.

Methanol/chloroform/water extracts were prepared from shoots of the plants from which xylem sap originated. Metabolic profiles of the polar phase of these extracts were recorded in negative ionization mode and examined for signals with $\mathrm{m} / \mathrm{z} 299$. Three signals were found, the intensity of one of which increased after infection. At the retention time of the 
metabolite generating this signal $(3.5 \mathrm{~min})$, another signal at $\mathrm{m} / \mathrm{z} 137$ was detected. Because 138 is the molecular weight of SA and the difference between 299 and 137 corresponds to a glucose residue, we hypothesized that the signal at $\mathrm{m} / \mathrm{z} 299$ originated from SAG and that the ion with $\mathrm{m} / \mathrm{z} 137$ originated from free SA generated during ionization by in-source fragmentation. The hypothesis was proven correct by comparing the xylem metabolite with $m / z 299$ with chemically synthesized SAG kindly provided to us by Prof. H. Kneifel (Forschungszentrum Jülich, Jülich, Germany). Comparison of retention times and analysis of product ion spectra on a triple quadrupole confirmed that the signal with $m / z$ 299, which was enhanced by the infection with $V$. longisporum, originated from SAG (Fig. 1).

\section{Relationship between salicylic acid and its glucoside in xylem sap and shoots and the effect of infection}

Because SAG is formed from SA by glucosyl transferase (Yalpani et al. 1992), we compared the levels of SA and SAG in xylem sap and shoot extracts. To this end, individual plants infected with $V$. longisporum at 28 dpi and uninfected controls of the same age were analyzed for the levels of SA and SAG in xylem sap and in acidic methanol/chloroform/water extracts of the shoots. Because SAG completely extracts into the polar phase while SA partitions between both phases (data not shown), the polar phase was used for the analysis. Quantitative analysis was performed by multiple reaction monitoring on a triple-quadrupole (transitions $\mathrm{m} / \mathrm{z} 299 \rightarrow \mathrm{m} / \mathrm{z} 137$ and m/z $299 \rightarrow \mathrm{m} / \mathrm{z}$ 93). Infected plants contained 0.1 to $1.5 \mu \mathrm{M}$ SAG in xylem sap and 2 to $189 \mathrm{nmol} \mathrm{g}^{-1}$ dry weight in shoots. Healthy plants only contained 0.06 to $0.11 \mu \mathrm{M}$ SAG in xylem sap and 0.51 to $1.48 \mathrm{nmol} \mathrm{g}^{-1}$ dry weight in shoots. The concentrations of salicylic acid and its glucoside were strongly correlated both in shoot tissue and xylem sap (Fig. 2a and 2b). The SAG levels in xylem sap and shoot extract were also correlated (Fig. 2c). These two correlations indicate that SA levels in the two compartments (xylem sap and shoot tissue) were also correlated, which was confirmed (data not shown).

In shoot extracts from infected plants, SAG levels were enhanced by a factor of 2.1 to 218 (median 76) relative to healthy controls. The increase of SAG in xylem sap of infected plants was 1.5 to 19.6 (median 6.1). In another experiment, SAG concentration in shoot extracts increased from 11- to 31-fold (median 17). 
Large differences in SA and SAG concentrations among individual infected plants indicated that SA and SAG were affected by the level of infection, which is notoriously variable (Eynck et al. 2007). We therefore investigated the relationships between SA and SAG concentrations, disease symptoms, and fungal DNA content in the tissue of inoculated plants. Xylem sap and shoot extracts from individual plants were collected 28 dpi. The results presented in Fig. 3 show that the elevation of SAG concentration both in xylem sap and shoots was correlated with disease severity assessed as reduction of shoot length. Both SAG and SA concentrations in shoots were highly correlated with the shoot length reduction caused by the infection (Fig. 3a and 3b). Similarly, a weak correlation between shoot length reduction and amount of SAG in xylem sap was observed (Fig. 3c); the same was true for SA (Fig. 3d).

Because disease symptoms are directly or indirectly caused by fungal colonization, we determined the amount of fungal DNA in hypocotyls of individual infected plants 28 dpi and compared quantities of DNA to those of SA and SAG. A strong correlation between SAG levels in shoot extracts from single plants and the amount of $V$. longisporum DNA was found (Fig. 4a). The relationship between disease symptoms (shoot length) and the amount of $V$. longisporum DNA in hypocotyls, however, was less pronounced (Fig. 4b).

\section{Changes in the concentration of SA, SAG, JA, and ABA in xylem sap at different times after infection}

Xylem sap was collected from plants 14, 21, 28, and 35 dpi. Because at early time points plants were too small to deliver sufficient amounts of xylem sap, each xylem sap sample at every time point represented a pool extracted from four plants. Salicylic acid glucoside, SA, JA, and ABA concentrations were determined by HPLC-MS in filtered xylem sap. For both healthy plants and $V$. dahliae-infected plants, SAG concentration remained at the same level (about $23 \mathrm{nM}$ ) over the course of the experiment. At all time points, SAG levels in xylem sap were significantly increased after infection with $V$. longisporum (Fig. 5a). The highest increase of 4.7-fold (up to $107 \mathrm{nM}$ ) was observed $14 \mathrm{dpi}$; thereafter SAG gradually declined to a 2.4 -fold increase at 35 dpi. The level of free SA in xylem sap in control plants reached the highest concentration at 14 dpi and decreased thereafter (Fig. $5 b)$. After infection with $V$. longisporum, the SA concentration increased and remained at about $500 \mathrm{nM}$ during the entire sampling period. The ratio of SA concentrations in 
$V$. longisporum-infected vs. healthy plants rose from 4.1 - to 9.1 between 14 dpi and 35 dpi because the gradual decrease of SA concentration was less pronounced in infected plants than in healthy plants. In xylem sap from $V$. dahliae-infected plants and healthy plants of the same age, SA concentrations were similar at all sampling times.

The level of JA in xylem sap continuously increased from 14 dpi to 35 dpi in $V$. longisporum-infected, V. dahliae-infected and healthy plants in the same way and the differences between infected and healthy plants were insignificant at all sampling times (Fig. 5c). Abscisic acid levels increased in the xylem sap of healthy, $V$. longisporum- and $V$. dahliae-infected plants in the same manner up to $28 \mathrm{dpi}$.

\section{Discussion}

The presence of SA and SAG in whole shoots, roots, fruits, and phloem sap of numerous plant species has been demonstrated, and the involvement of SA in plant defense response is well established (Loake and Grant 2007). High concentrations of SA in phloem sap after pathogen attack (Métraux et al. 1990; Rasmussen et al. 1991) and experiments with exogenously applied radioactively labeled SA inspired the view that SA is also transported within the xylem, but attempts to detect SA in xylem sap by HPLC with spectrophotometric methods failed (Rocher et al. 2006). We identified and quantified SA and SAG in xylem sap of B. napus by HPLC with mass spectrometry. The failure of the previous attempt can be explained by lower sensitivity of the detector and by the dilution of xylem sap before analysis.

Salicylic acid glucoside is formed by the activity of glucosyl transferase, which has been characterized in tobacco (Enyedi and Raskin 1993), oat roots (Yalpani et al. 1992), soybeans (Dean et al. 2003), and Arabidopsis thaliana (Song 2006). Salicylic acid glucoside is synthesized from SA in a concentration-dependent manner (Dean et al. 2005) and the glucosylation of SA is reversible (Hennig et al. 1993). It is therefore not surprising that we found tight correlations between the levels of SAG and SA both in the shoot tissue and in xylem sap (Fig. 2a-c).

Active transport of SAG into the vacuole has been demonstrated (Dean and Mills 2004; Dean et al. 2005), but little is known about the transport of SAG into other organelles and 
compartments or its long-term transfer. The accumulation of SAG in inoculated leaf tissue but not in phloem sap and uninoculated leaves led Enyedi et al. (1992) to hypothesize that SAG was not the translocatable form of SA. Our detection of SA and SAG in xylem sap, however, corroborates the assumption of Rocher et al. (2006) that SA may be rapidly transported upwards by the transpiration stream. The same apparently holds true for SAG. Upward transfer with xylem sap has been established for the translocation of abscisic acid from the roots, where it is synthesized under drought stress, to its target in the shoots (Hartung et al. 2002).

Our data show that SA, both as free acid and in form of a glucoside, can be transported over long distances within the vascular tissue. This prompts the question of how this finding can be reconciled with the current view of the role of SA in plant defense. Many experiments have shown that a mobile signal of unknown nature carries information about pathogen attack from lower parts of the plant to its upper parts. For example, in a typical experiment on tobacco, the lower leaves are TMV-inoculated and the upper leaves respond by launching a defense reaction, which involves the accumulation of SA (Shulaev et al.1995). While the transport of SA in phloem was demonstrated (Métraux et al. 1990), such transport cannot fully explain the distribution of ${ }^{14} \mathrm{C}$-labeled SA or the pattern of defense induction, as discussed by Rocher et al. (2006). The latter authors suggested that distribution of SA via xylem might play a role in defense response to pathogen attack. That SA and SAG move through xylem and that such movement is part of a defense response is supported by the current findings: SA and SAG are present in xylem sap of B. napus and their concentrations in xylem sap increase after infection. Root-to-shoot signaling also appears to occur in $A$. thaliana infected with $V$. longisporum, because certain genes in the shoot are induced by infection long before the fungus can be detected in this part of the plant (Tappe et al. 2008). While Johansson et al. (2006) suggested that SA is involved because they observed the induction of SA-dependent pathogenesis-related $(P R)$ genes $P R-1$ and $P R-2$ in in vitro-grown $A$. thaliana as early as 7 dpi with $V$. longisporum, Tappe et al. (2008) did not detect any increase in SA levels in soil-grown A. thaliana until 35 dpi. We observed a correlation between shoot length reduction and SA/SAG concentrations in B. napus infected with $V$. longisporum. Salicylic acid and SAG levels in xylem sap after infection increased as compared to uninfected controls at all time points surveyed (14, 21, 28, 35 dpi). Severely diseased plants with high levels of SA and SAG contained high amounts of fungal DNA in the hypocotyls. At 28 dpi, fungal biomass and shoot length 
were negatively correlated. Both fungal biomass and shoot length were strongly correlated with the SAG levels (Fig. 3a and 4). Apparently, the presence of $V$. longisporum in the hypocotyl dramatically enhanced SA and SAG levels in the remaining part of the shoot. As early as 14 dpi, stunting was visible, fungal DNA was detected in the hypocotyls (data not shown), and SA and SAG levels in xylem sap were elevated (Fig. 5a, b). Whereas our data with $B$. napus indicate that SA is involved in plant defense against $V$. longisporum, Arabidopsis genotypes impaired in the SA pathway (NahG, sid2-1, eds1-1, pad4-1) did not exhibit enhanced susceptibility to V. longisporum (Johansson et al. 2006). Interestingly, all of these mutants showed an increase in fresh weight after inoculation as compared to the wild type. Two of the mutants (sid2-1 and pad4-1) were even found to be less affected than the wild type by $V$. longisporum with regard to disease symptoms. In contrast to B. napus, it appears that the SA pathway in A. thaliana does not play an important role in $V$. longisporum infection, and that A. thaliana might not be an appropriate model for the $V$. longisporum-oilseed rape pathosystem.

$V$. dahliae-infected plants were indistinguishable from uninfected controls both in SA and SAG levels and in phenotype (Fig. 5a, b). Veronese et al. (2003) showed that $P R-1$ was not induced in in vitro-grown $A$. thaliana by $V$. dahliae, but the strain they used (VdBob.70 (90-02)), which was isolated from cauliflower, appears to be $V$. longisporum (A. von Tiedemann, unpublished results). Tjamos et al. (2005) observed increased expression of $P R-1, P R-2$, and $P R-5$ in A. thaliana grown in soil after infection with $V$. dahliae. Most recently, the lack of disease symptoms in $B$. napus infected with $V$. dahliae was demonstrated by Eynck et al. (2007) confirming the non-host relationship of this Verticillium species on Brassicaceae (Zeise and von Tiedemann, 2002).

Jasmonic acid and ABA levels in A. thaliana increased in response to $V$. longisporum infection at late stages of infection (Tappe et al. 2008). Johansson et al. (2006) demonstrated that the JA pathway was decisively involved in the response of $A$. thaliana to $V$. longisporum and that $\mathrm{ABA}$ was not required (though the $A B A 2$ mutant was impaired in defense reaction). In our work with B. napus, JA and ABA levels in xylem sap were similar in $V$. longisporum-infected plants, in $V$. dahliae-infected plants, and in uninfected controls, indicating that $\mathrm{JA}$ and $\mathrm{ABA}$ are not involved in the response of $B$. napus to $V$. longisporum infection. The importance of SA, JA and ABA for defense against $V$. longisporum infection apparently differs among plants. 
In summary, this work demonstrates for the first time the presence of SA and SAG in xylem sap. With the transpiration stream, these molecules can be transported from roots to the shoots and thus might act as long-distance signals in the plant. The levels of SA and SAG in xylem sap of B. napus are enhanced by $V$. longisporum infection and are correlated with disease symptoms and fungal biomass, strongly indicating that SA is involved in the response of $B$. napus to $V$. longisporum infection. The role of xylem transport of SA and SAG in plant defense signaling in general and in the specific pathosystem studied here remains an issue for future research.

\section{Acknowledgements}

This research work was part of an interfacultative research unit FOR546 funded by Deutsche Forschungsgemeinschaft (DFG), Bonn, Germany. We acknowledge the assistance of P. Bartoschek, M. Bossmann, I. Kunze, and U. Vetterling with xylem sap extraction.

\section{References}

Birkemeyer C, Kopka J (2007) Design of metabolite recovery by variations of the metabolite profiling protocol. In: Nikolau BJ, Wurtele ES (ed) Concepts in Plant Metabolomics. Springer, Dordrecht, The Netherlands, pp 45-69

Brandfass C, Karlovsky P (2006) Simultaneous detection of Fusarium culmorum and F. graminearum in plant material by duplex PCR with melting curve analysis. BMC Microbiol 6:4

Dean JV, Shah RP, Mohammed LA (2003) Formation and vacuolar localization of salicylic acid glucose conjugates in soybean cell suspension cultures. Physiol Plant 118:328-336 
Dean JV, Mills JD (2004) Uptake of salicylic acid 2-O-b-D-glucose into soybean tonoplast vesicles by an ATP-binding cassette transporter-type mechanism. Physiol Plant 120:603612

Dean JV, Mohammed LA, Fitzpatrick T (2005) The formation, vacuolar localization, and tonoplast transport of salicylic acid glucose conjugates in tobacco cell suspension cultures. Planta 221:287-296

Durrant WE, Dong X (2004) Systemic acquired resistance. Annu Rev Phytopathol 42:185209

Enyedi AJ, Yalpani N, Silverman P, Raskin I (1992) Localization, conjugation, and function of salicylic acid in tobacco during the hypersensitive reaction to tobacco mosaic virus. Proc Natl Acad Sci U S A 89:2480-2484

Enyedi AJ, Raskin I (1993) Induction of UDP-glucose: Salicylic acid glucosyltransferase gctivity in tobacco mosaic virus-inoculated tobacco (Nicotiana tabacum) leaves. Plant Physiol 101:1375-1380

Eynck C, Koopmann B, Grunewaldt-Stoecker G, Karlovsky P, von Tiedemann A (2007) Differential interactions of Verticillium longisporum and V. dahliae with Brassica napus detected with molecular and histological techniques. Eur J Plant Pathol 118:259-274

Gaffney T, Friedrich L, Vernooij B, Negrotto D, Nye G, Uknes S, Ward E, Kessmann H, Ryals J (1993) Requirement of salicylic acid for the induction of systemic acquired resistance. Science 261:754-756

Hartung W, Sauter A, Hose E (2002) Abscisic acid in the xylem: where does it come from, where does it go to? J Exp Bot 53:27-32

Hennig J, Malamy J, Grynkiewicz G, Indulski J, Klessig DF (1993) Interconversion of the salicylic acid signal and its glucoside in tobacco. Plant J 4:593-600 
Johansson A, Staal J, Dixelius C (2006) Early responses in the Arabidopsis-Verticillium longisporum pathosystem are dependent on NDR1, JA- and ET-associated signals via cytosolic NPR1 and RFO1. Mol Plant Microbe Interact 19:958-969

Kiefer IW, Slusarenko AJ (2003) The pattern of systemic acquired resistance induction within the Arabidopsis rosette in relation to the pattern of translocation. Plant Physiol $132: 840-847$

Loake G, Grant M (2007) Salicylic acid in plant defence-the players and protagonists. Curr Opin Plant Biol10:466-472

Maldonado AM, Doerner P, Dixon RA, Lamb CJ, Cameron RK (2002) A putative lipid transfer protein involved in systemic resistance signalling in Arabidopsis. Nature 419:399403

Métraux JP, Signer H, Ryals J, Ward E, Wyss-Benz M, Gaudin J, Raschdorf K, Schmid E, Blum W, Inverardi B (1990) Increase in salicylic acid at the onset of systemic acquired resistance in cucumber. Science 250:1004-1006

Mölders W, Buchala A, Metraux JP (1996) Transport of salicylic acid in tobacco necrosis virus-infected cucumber plants. Plant Physiol 112:787-792

Nandi A, Welti R, Shah J (2004) The Arabidopsis thaliana dihydroxyacetone phosphate reductase gene SUPPRESSOR OF FATTY ACID DESATURASE DEFICIENCY1 is required for glycerolipid metabolism and for the activation of systemic acquired resistance. The Plant Cell 16:465-477

Park SW, Kaimoyo E, Kumar D, Mosher S, Klessig DF (2007) Methyl salicylate is a critical mobile signal for plant systemic acquired resistance. Science 318:113-116

Rasmussen JB, Hammerschmidt R, Zook MN. (1991) Systemic induction of salicylic acid accumulation in cucumber after inoculation with Pseudomonas syringae pv syringae. Plant Physiol 97:1342-1347 
Rocher F, Chollet JF, Jousse C, Bonnemain JL (2006) Salicylic acid, an ambimobile molecule exhibiting a high ability to accumulate in the phloem. Plant Physiol 141:16841693

Scholander PF, Hammel HT, Bradstre ED, Hemmings E A (1965) Sap pressure in vascular plants - Negative hydrostatic pressure can be measured in plants. Science 148:339-346

Shulaev V, Leon J, Raskin I (1995) Is salicylic acid a translocated signal of systemic acquired resistance in tobacco? Plant Cell 10:1691-1701

Shulaev V, Silverman P, Raskin I (1997) Airborne signalling by methyl salicylate in plant pathogen resistance. Nature 385:718-721

Smith CA, Want EJ, O`Maille G, Abagyan R, Suizdak G (2006) XCMS: processing mass spectrometry data for metabolite profiling using nonlinear peak alignment, matching, and identification. Anal Chem 78:779-787

Song JT (2006) Induction of a salicylic acid glucosyltransferase, atsgt1, is an early disease response in Arabidopsis thaliana. Mol Cells 22:233-238

Tappe H, Floerl S, Thurow C, Carsjens JG, Ratzinger A, Dilcher M, Karlovsky P, Feussner I, Polle A, Gatz C (2008) Expression profiling of Arabidopsis thaliana plants after infection with the xylem-colonizing fungus Verticillium longisporum. Submitted.

Tjamos SE, Flemetakis E, Paplomatas EJ, Katinakis P (2005) Induction of resistance to Verticillium dahliae in Arabidopsis thaliana by the biocontrol agent K-165 and pathogenesis-related proteins gene expression. Mol Plant Microbe Interact 18:555-561

Truman W, Bennett MH, Kubigsteltig I, Turnbull C, Grant M (2007) Arabidopsis systemic immunity uses conserved defense signaling pathways and is mediated by jasmonates. Proc Natl Acad Sci U S A 104:1075-1080 
Vernooij B, Friedrich L, Morse A, Reist R, Kolditz-Jawhar R, Ward E, Uknes S, Kessmann H, Ryals J (1994) Salicylic acid is not the translocated signal responsible for inducing systemic acquired resistance but is required in signal transduction. Plant Cell 6:959-965

Veronese P, Narasimhan ML, Stevenson RA, Zhu JK, Weller SC, Subbarao KV, Bressan RA (2003) Identification of a locus controlling Verticillium disease symptom response in Arabidopsis thaliana. Plant J 35:574-587.

Yalpani N, Silverman P, Wilson TM, Kleier DA, Raskin I (1991) Salicylic acid is a systemic signal and an inducer of pathogenesis-related proteins in virus-infected tobacco. Plant Cell 3:809-818

Yalpani N, Balke NE, Schulz M (1992) Induction of UDP-glucose: salicylic acid glucosyltransferase in oat roots. Plant Physiol 100:1114-1119

Yazaki K, Sugiyama A, Morita M, Shitan N (2007) Secondary transport as an efficient membrane transport mechanism for plant secondary metabolites. Phytochem Rev DOI $10.1007 / \mathrm{s} 11101-007-9079-8$

Zeise K, von Tiedemann A (2001) Morphological and physiological differentiation among vegetative compatibility groups of Verticillium dahliae in relation to Verticillium longisporum. J Phytopathology 149:469-475

Zeise K, von Tiedemann A (2002) Host specialization among vegetative compatibility groups of Verticillium dahliae in relation to V. longisporum. J Phytopathology 150:112119.

Zhou L, Hu Q, Johansson A, Dixelius C (2006) Verticillium longisporum and Verticillium dahliae: infection and disease in Brassica napus. Plant Pathol 55:137-144 


\section{Figures}

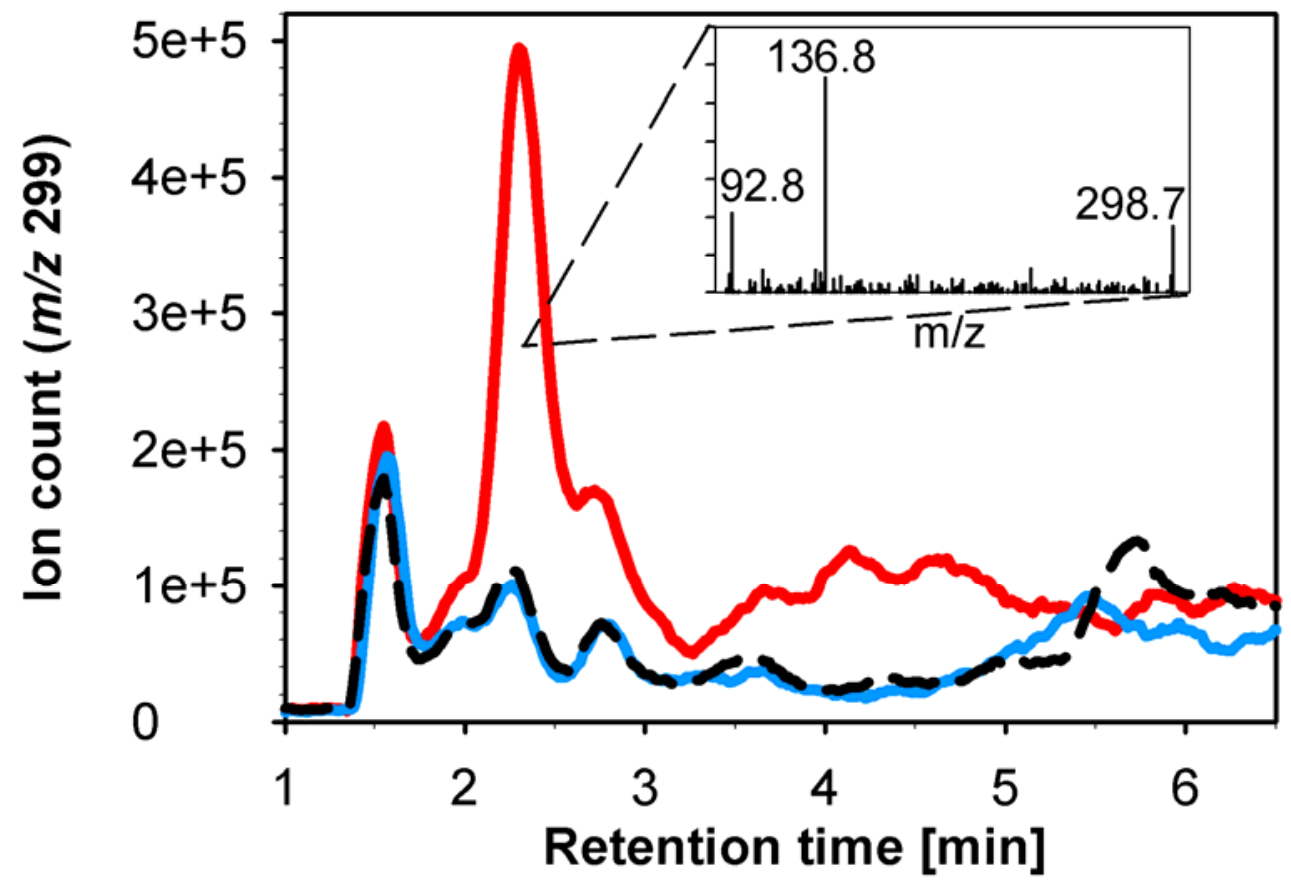

Figure 1: Selected ion chromatogram of salicylic acid glucoside in xylem sap

Xylem sap of healthy plants (black dashed line), V. longisporum-infected plants (red line) and of $V$. dahliae-infected plants (blue line) was analyzed by HPLC-MS in selected ion monitoring mode. Ion chromatograms of salicylic acid glucoside $\left(\mathrm{m} / \mathrm{z} 299,[\mathrm{M}-\mathrm{H}]^{-}\right)$and product ion spectra of $m / z 299$ are shown. 

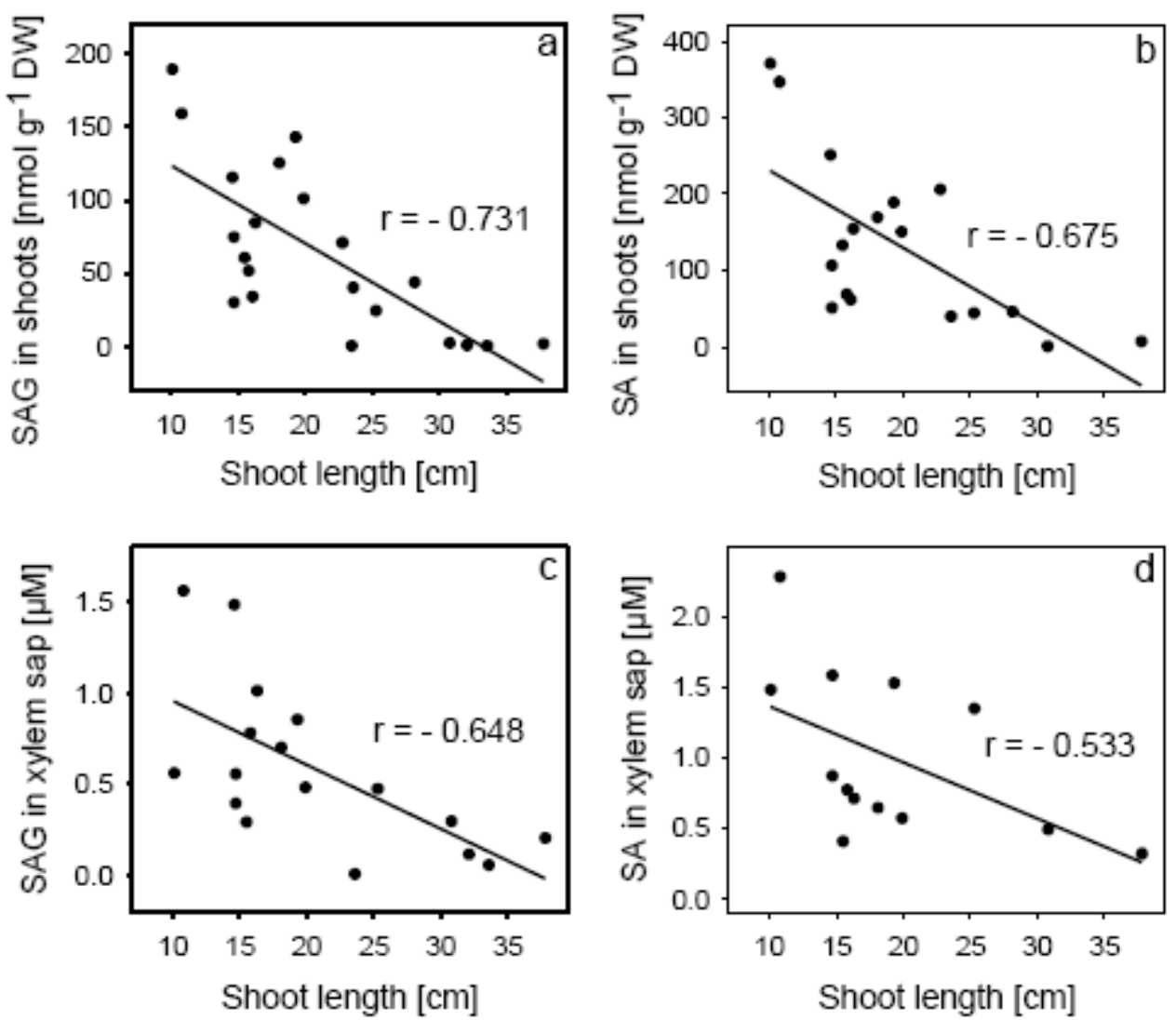

Figure 2: Comparison of the concentrations of salicylic acid and salicylic acid glucoside in xylem sap and shoot extracts

Xylem sap from $V$. longisporum-infected single plants 28 dpi was analyzed. Pearson correlation is given ( $\mathrm{r}$ ), all correlations are significant at $\mathrm{P} \leq 0.01$ (1-tailed $\mathrm{t}$-test). 

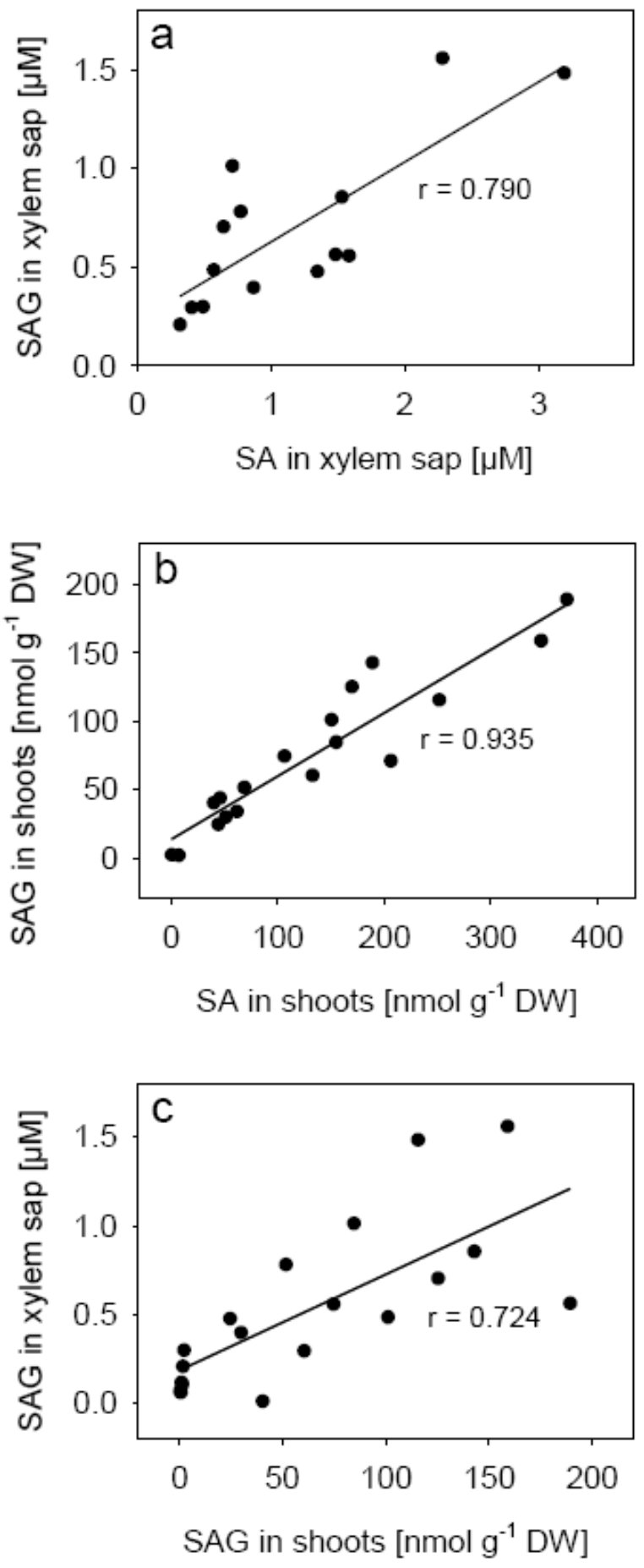

Figure 3: Concentrations of salicylic acid and salicylic acid glucoside in xylem sap and shoot extracts in relation to shoot length

Xylem sap from $V$. longisporum-infected single plants 28 dpi was analyzed. Pearson correlation is given (r), all correlations are significant at $\mathrm{P} \leq 0.05$ (1-tailed t-test). 

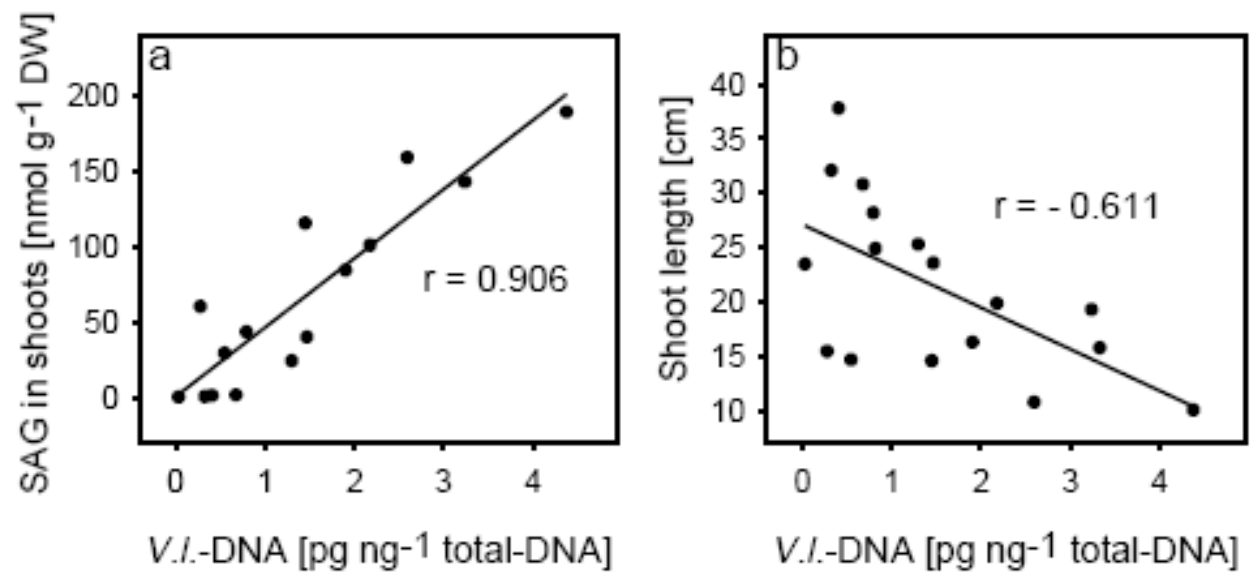

Figure 4: Concentrations of salicylic acid and salicylic acid glucoside in shoot extracts in relation to the amount of fungal DNA in the hypocotyl

Shoot extracts from individual $V$. longisporum-infected plants 28 dpi were analyzed; fungal DNA (V.l.-DNA) in hypocotyl was determined by real-time PCR. Pearson correlation is given $(r)$, all correlations are significant at $\mathrm{P} \leq 0.01$ (1-tailed t-test). 

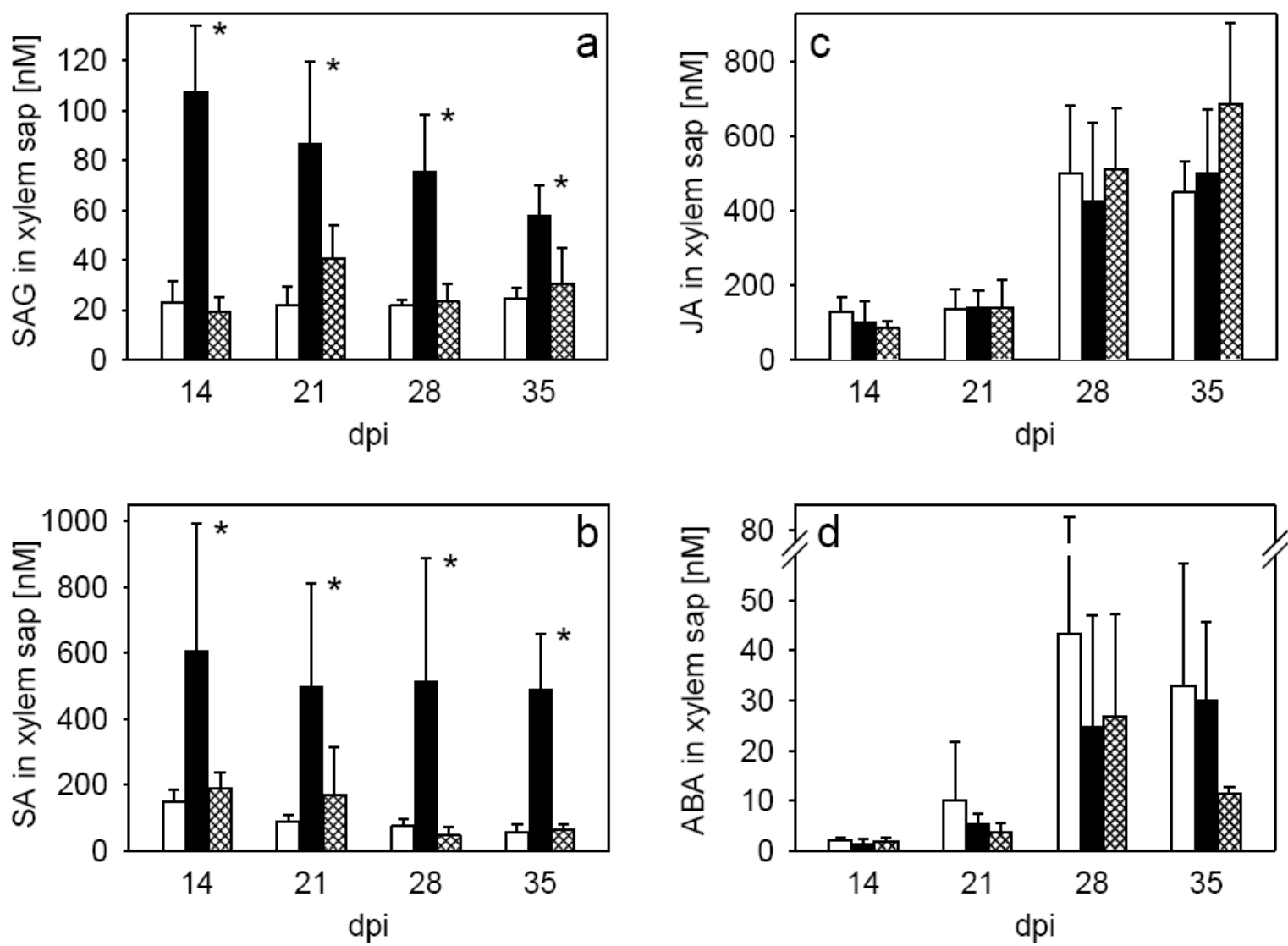

Figure 5: Concentrations of SAG, SA, JA and ABA in xylem sap of $V$. longisporumand $V$. dahliae-infected $B$. napus plants

Analysis of xylem sap from healthy plants (white), V. longisporum-infected plants (black) and $V$. dahliae-infected plants (patterned) at different time points after infection is shown. Each data point represents an average of 5 samples (each sample pooled from 4 plants). Error bars indicate standard deviation. Values showing significant differences $(\mathrm{P} \leq 0.05)$ between xylem sap samples from $V$. longisporum-infected plants and both remaining samples (mock- and $V$. dahliae-infected) are labeled by asterisks. a) Salicylic acid glucoside, b) salicylic acid, c) jasmonic acid, d) abscisic acid. 


\title{
Chapter 4: Relationship between metabolic and genomic diversity in sesame (Sesamum indicum L.)*
}

*This manuscript has been accepted for publication in BMC Genomics.

Hernán Laurentin ${ }^{1}$, Astrid Ratzinger ${ }^{2}$ and Petr Karlovsky ${ }^{2}$

${ }^{1}$ Universidad Centroccidental Lisandro Alvarado, Biologic Sciences Department, Agronomy Faculty, Barquisimeto, Venezuela.

${ }^{2}$ University Goettingen, Molecular Phytopathology and Mycotoxin Research Unit, Goettingen, Germany.

\begin{abstract}
Background

Diversity estimates in cultivated plants provide a rationale for conservation strategies and support the selection of starting material for breeding programs. Diversity measures applied to crops usually have been limited to the assessment of genome polymorphism at the DNA level. Occasionally, selected morphological features are recorded and the content of key chemical constituents determined, but unbiased and comprehensive chemical phenotypes have not been included systematically in diversity surveys. Our objective in this study was to assess metabolic diversity in sesame by nontargeted metabolic profiling and elucidate the relationship between metabolic and genome diversity in this crop.
\end{abstract}

\section{Results}

Ten sesame accessions were selected that represent most of the genome diversity of sesame grown in India, Western Asia, Sudan and Venezuela based on previous AFLP studies. Ethanolic seed extracts were separated by HPLC, metabolites were ionized by 
positive and negative electrospray and ions were detected with an ion trap mass spectrometer in full-scan mode for $\mathrm{m} / \mathrm{z}$ from 50 to 1000 . Genome diversity was determined by Amplified Fragment Length Polymorphism (AFLP) using eight primer pair combinations. The relationship between biodiversity at the genome and at the metabolome levels was assessed by correlation analysis and multivariate statistics.

\section{Conclusions}

Patterns of diversity at the genomic and metabolic levels differed, indicating that selection played a significant role in the evolution of metabolic diversity in sesame. This result implies that when used for the selection of genotypes in breeding and conservation, diversity assessment based on neutral DNA markers should be complemented with metabolic profiles. We hypothesize that this applies to all crops with a long history of domestication that possess commercially relevant traits affected by chemical phenotypes.

\section{Background}

The diversity of characters among members of a species is an inherent feature of biological complexity. Most studies of biological diversity in crops have focused on morphological characters and DNA markers, covering both ends of the path of gene expression from genome to phenotype. Genome analysis records and compares the genetic make-up of lineages or individuals based on DNA sequences or fragment patterns. Both sequence analysis and DNA fingerprinting sample genome diversity, which is independent of environmental conditions and the developmental stage of the organism [1]. AFLP markers [2] are anonymous and are generally thought to be selectively neutral, which probably holds true for many kinds of DNA markers [3]. Even whole-genome sequencing of populations, the ultimate genome diversity survey tool, reveals at most the potential of a population to express various phenotypic features. Approaches based on transcriptomics and proteomics can identify gene expression patterns that underlie the current phenotype and that are affected by environment and the developmental stage of the organism. The relationship between the abundance of mRNA and protein molecules on one side and of phenotypic features relevant for crop production on the other is obscure and cannot yet be exploited for breeding purposes even in major crops with extensive genomic resources, let alone in minor or orphan crops. 
A third level of gene expression, represented by the metabolic constitution of the organism, is directly related to features that are important in plant production. We are interested in secondary metabolites, because these natural products provide most of the chemical diversity in plants, and are a key factor (i) affecting the resistance of crops to pathogens and pests, and (ii) controlling commercially relevant traits such as taste, color, aroma and antioxidative properties.

The metabolic phenotype of an organism is analyzed by metabolomics, whose final goal is to identify and quantify all of the metabolites present in a sample $[4,5]$. Such a complete inventory is not attainable with current technology even for model organisms, so different types of metabolite analysis with more limited scopes serve as surrogates. Metabolic fingerprints are a static set of analytical signals originating from small molecules (e.g. HPLC peaks, TLC spots, or mass spectra), which can be used for diagnostic purposes or to confirm the origin of a sample. In metabolic profiling, which is analogous to transcription profiling, metabolic signals, either anonymous or assigned to structures, are generated and evaluated quantitatively for samples originating from different varieties, physiological states or treatments. Term profiling is also used for a comprehensive analysis of a class of substances defined by common structural features (e.g., oxylipin profiling). Alternative definitions of metabolic profiling and fingerprinting $[6,7]$ are likely to lead to confusions whenever metabolic analysis and genome fingerprinting are treated jointly.

Sesame (Sesamum indicum L.) is one of the most ancient crops $[8,9]$. Sesame seed is highly nutritive (50\% oil and $25 \%$ protein) and may be consumed directly or pressed to five an oil of excellent quality. Most studies of secondary metabolites in sesame focused on the lignans sesamin, sesamol, sesamolin and sesaminol $[10,11,12,13]$ in seeds. These natural products have antioxidative properties and may confer health-promoting qualities on products containing sesame seeds or oil $[14,15,16,17]$. Sesame lignans also may play a role in the resistance of sesame to insect pests and microbial pathogens $[18,19,20,21$, $22,23]$. The metabolism of sesame lignans after ingestion is understood to a limited extent [24]. Metabolic profiling has not been a part of diversity studies in sesame.

Our objective in this study was to compare metabolic and genomic diversity in sesame and to discern the relationship between the two sets of data. Based on the difference in the diversification of sesame at the genomic and metabolic levels we will assess the usefulness of metabolic profiles in the identification of parent lines for breeding programs and in the selection of accessions for biodiversity preservation in sesame. 


\section{Results}

Sesame accessions for this study were selected based on previously published AFLP data and represent most of the genome diversity in sesame from India, Western Asia, Sudan and Venezuela. Among these accessions, eight accessions have Jaccard similarity coefficients from pairwise comparisons that range from 0.39 to 0.85 . These accessions encompass nearly all of the genome diversity detected by AFLP in the two-dimensional space of principal coordinate analysis and represent the four previously described major clusters [25]. The two Venezuelan genotypes, an experimental line and a commercial cultivar, were included because they represent Venezuelan breeding products with a Jaccard's similarity coefficient of 0.45 [26]. These genotypes represent the two major clusters comprising Venezuelan commercial cultivars and contain $80 \%$ of the total genetic diversity of sesame in Venezuela.

Three hundred and eighty one AFLP markers, ranging from 100 to 550 base-pairs, were recorded using 8 primer combinations. Ninety-five percent of the markers were polymorphic. Eighty-eight bands (23\%) were unique, ranging from 5 (Turkey) to 21 (India 7) per accession (Table 1).

The reproducibility of the metabolic analysis was very good because similarity and dissimilarity measures and principal component analysis results showed negligible differences regarding three independent profiles generated from extracts of Sudan3 accession and compared to the other 9 accessions. The average of the three replicas obtained for Sudan3 was used for all further analysis.

Eighty-eight dominant metabolic signals were selected based on the mass chromatogram quality index, 47 of them in negative mode ESI and 41 in positive mode ESI. More than $50 \%$ of the signals resulted from peaks eluting in a well-resolved area with retention times between 15 and $27 \mathrm{~min}$. Thirty-four signals were common to all accessions, 16 in positive mode ESI and 18 in negative mode ESI. Eight signals were either accession-specific or present in all except one accession (Table 2). No association was found between the distribution of unique AFLP markers and accession-specific metabolic signals.

The coefficient of correlation between correlation coefficient-based similarity matrix and simple-matching coefficient-based similarity matrix was $0.63(\mathrm{P}<0.01)$. Correlation between matrices obtained from AFLP data and metabolic profiles was not significant. Comparisons of matrices of metabolic data with Jaccard's coefficient matrix of AFLP data resulted in a correlation coefficient of $-0.09(\mathrm{P}<0.33)$ for the simple matching coefficient 
matrix and $-0.24(\mathrm{P}<0.18)$ for the correlation matrix. There were consistencies in scatter plots for pairs of accessions that fell into the same category (high similarity, intermediate similarity, or low similarity) for genomic and metabolic data (Fig. 1). Accession pair SyriaSudan 3 had high similarity on both axes, Sudan2-43x32, India7-43×32, and India5-Sudan2 were dissimilar both in metabolic profiles and AFLP fingerprints and pairs India1-India8, India1-Syria, India1-Turkey, India1-43x32 and India8-Turkey had intermediate similarities.

Biplot of principal coordinate analysis based on AFLP data calculated from Jaccard's coefficient captured $64 \%$ of the total variation (Fig. 2). Accessions Sudan2 and India7 on one side, and commercial cultivars Inamar and $43 \times 32$ on the other, are the most distinctive. Biplots of principal component/coordinate analysis based on correlation coefficient, which captured $62 \%$ of the variation, and simple matching coefficient, which captured $77 \%$ of the variation, had similar patterns in that accessions India5 and $43 \times 32$ formed one group and the remaining eight accessions formed a second group. Visual comparison of biplots obtained with AFLP and metabolic profiles confirmed the classification of cultivar $43 \times 32$ as the most distinctive, which explains why Sudan2-43x32 was one of the most dissimilar pairs. Both biplots grouped Syria-Sudan3 as the most similar pair and India1-Syria, India1Sudan3, India1-Turkey and I8-43×32 as pairs with intermediate similarities. The most important contradiction between both biplots was the placement of India5, India7 and Inamar. Based on AFLPs, India7 and Inamar were the most distinctive accessions, whereas metabolic profiles grouped them together with six other accessions. The opposite situation was found for India5, which was classified as one of the most distinctive based on metabolic profiles, but groups together with 5 other accessions based on the AFLP data.

\section{Discussion}

Seed metabolic profiles were unrelated to the geographic origin of the accessions studied, which is similar to results obtained previously for genome diversity as assessed by AFLPs [25]. The relationship patterns generated for the AFLP data and for the seed metabolic profiles were different. No relevant data from other plant species are available for comparison, but there are two studies of the relationship between genomic and metabolic diversity in microorganisms. In rhizobia (bacteria), metabolic and genomic data (AFLP) 
were unrelated [27], while there were strong similarities between genome variation and metabolite diversity between two endophytic fungi [28].

If the number of characters reflects the sampling depth, then the metabolic profiles and AFLP fingerprints cover only a small portion of the underlying character sets. The AFLPbased analysis appears more robust because it was based on 363 polymorphic bands while only 88 signals were evaluated in the metabolic profiles. However, the metabolic profiles may contain more information because they are based on continuous rather than binary variables. To test this hypothesis we transformed the metabolic data into a binary matrix and compared the binary and continuous results. The quantitative information (normalized amplitudes of mass signals) did not affect the similarity patterns and therefore can be neglected in diversity surveys.

Diversity in AFLP patterns and metabolic profiles reflect different facets of genomic polymorphism. AFLPs are insensitive to gene expression and may occur most frequently in noncoding portions of the genome. Seed metabolic profiles result from biosynthetic activities in embryo and endosperm based on the expression of a small fraction of the total genomes. If the samples are representative, then differences between the diversity patterns are due to differences in the diversification of sesame at genomic and metabolic levels. Because the majority of plant genomes consist of noncoding sequences, most changes in AFLP patterns are expected to result from neutral mutations fixed by genetic drift rather than by selection. On the other hand all metabolites synthesized by a plant affect its fitness: apart from the metabolic costs incurred, anabolic processes are subjected to different selection pressures, both positive (e.g., resistance to pathogens, protection against light, improved dissemination of seeds) and negative (e.g., reduced attractiveness of seeds for disseminating animals because of a bitter taste, volatiles attracting pests, trigger of the germination of microbial pathogens). The synthesis of many secondary metabolites is known to be limited to conditions under which they enhance the fitness of their producer, limiting the costs of biosynthesis [41]. Metabolic profiles of sesame recorded under different environmental conditions are therefore likely to differ. For example, exposure to biotic stress is likely to generate defence-related signals, which may not be present in metabolic profiles of plants grown in the absence of pathogens and pests. Regardless of the progress in analytical technologies, chemical diversity revealed by metabolic profiling under a single set of conditions therefore remains an underestimate of the total metabolic capacity of sesame. 
The genetic basis of the variation in the metabolic composition on plants was proven by the association between metabolic peaks detected by HPLC-MS and specific genomic loci in segregating populations of A. thaliana [29]. The disparity between the diversity patterns represented by AFLPs and by metabolic profiles thus provides insights into the processes that led to the composition of the current sesame genome. With the growing availability of instrumentation and software tools for nontargeted metabolite analysis by HPLC-MS [30, 31], large-scale metabolic profiling is becoming a feasible task for diversity studies in cultivated plants. From a practical point of view, crop improvement programs [32] will benefit from the complementation of diversity assessment based on DNA markers by metabolic profiling particularly for plants such as pepper [35, 40], mulberry [36, 37, 39] and fenugreek [38], the commercial value of which is largely affected by complex mixtures of secondary metabolites.

In addition to genuine differences in similarity patterns between genomic and metabolomic profiles caused by differences in diversification rates, non-representative sampling also may lead to inconsistencies. The involvement of one accession in many pairwise comparisons would amplify this distortion. For example, two accessions in our set affect 17/45 pairwise comparisons. Thus a small number of biased data sets may alter the global pattern of biplots in a principal components or coordinates analysis. In this situation, scatter plots can identify which data sets are correlated, which are not, and which are not independent. Consistencies in scatter plots corroborate the representativeness of sampling in a particular pairwise comparison. For example, the pairs Sudan2-43x32, India7-43×32 and India5-Sudan2 were consistently the most dissimilar pairs in both the AFLP and the metabolic analysis. Similarly consistent were the comparison of pairs Syria-Sudan3 (highly similar for both approaches), and India1-Syria, India1-India8, India1-Turkey, India1-43x32 and I8-Turkey (intermediate similarity). Thus, the consistency of pairwise comparisons is independent of the similarity level.

Selection on the metabolome of a plant could distort the congruency in diversification between neutral DNA markers (AFLP) and metabolic profiles in a manner dependent on the intensity and duration of the selection pressure. Comparative analysis of intra- and interpopulation diversity at the genomic and metabolic levels will aid our understanding of the effect of selection on the evolution of metabolic capacity. Dedicated statistical tools that test the congruency in diversification of the metabolome and the genome are not 
available. However, tools for diversity estimation established in population genetics can be applied, offering at least qualitative insights.

Plants subjected to selection for metabolic traits should evolve faster on the metabolic level than neutral DNA markers should at the genomic level, as the rate of fixation of neutral mutations is controlled by only the mutation rate and population size. For example, Turkey-Syria accession pair appears to demonstrate the effect of selection on metabolic profiles of sesame (compare Fig. 2 and 3).

Common selection pressure exerted on different genotypes may result in different outcomes, i.e. convergent evolution or increased diversification. Increased diversification occurs when the biochemical basis of traits under selection differs among genotypes, e.g. when unrelated metabolic pathways enhance resistance to a common pathogen. The accession pair India7-Syria are very different at the genomic level but have similar metabolic phenotypes, and could have resulted from convergent evolution driven by common selection on genotypes with the same metabolic potential. Alternatively, neutral markers may have diversified over a long period of time, during which the metabolic phenotype was maintained by constant selection pressure.

The third situation encountered in our comparison of genomic and metabolic diversity in sesame was that the relative amount of diversification between the members of a pair was qualitatively similar at both the genomic and the metabolic levels. Thus pairs that were highly different at the genomic level also were highly different at the metabolic level. We suggest that varying selection and complex evolutionary histories might explain this kind of data. The analysis of the inheritance of metabolic patterns and of the association between metabolic and genomic markers might provide deeper insights. We have begun to generate segregating populations to address these questions.

The purpose of untargeted metabolic profiling in our work was to sample metabolic diversity without bias for the biological activity or practical relevance of the underlying compounds. One might want to know, however, whether metabolites of particular interest have been recovered in ethanol extracts used for the analysis. The most prominent metabolites of sesame are phenylpropanoids with one or more methylenedioxybenzole (piperonyl-) moieties such as sesamin and sesaminol. These lignans occur in free form and as di- and triglucosides and possess antioxidative properties. Certain sesame lignans lower blood and liver cholesterol levels, qualifying as health-promoting agents. In traditional analytical protocols, crushed sesame seeds are defatted with hexane prior extraction with 
ethanol or methanol. The defatting step is often used in lignan analysis in order to improve the recovery [42], but the lignans of sesame can be extracted from oil directly into methanol [43, 44], indicating that defatting is not necessary. Indeed, an HPLC method for the analysis of sesame lignans based on extraction with $80 \%$ ethanol without defatting was described [45]. Similarly, extraction of sesame with methanol without defatting was used for sesamin determination [46]. In line with these results, we observed that the recovery of eight sesame lignans did not improve substantially by defatting seeds prior ethanol extraction (data not shown), which we used in the comparison of lignan content among sesame accessions [47]. As long as the life span of reverse phase columns is not a matter of concern, defatting seeds prior extraction can be omitted.

\section{Conclusions}

Diversity patterns in sesame (Sesamum indicum L.) at the genomic level (neutral DNA markers) and at the metabolic level (nontargeted HPLC-MS profiles) differed, often showing a higher diversification rate at the metabolic level. For sesame breeders this means that the distances among accessions determined by genome fingerprinting need not reflect differences in metabolic capacity. Genetic analyses based on neutral markers is not an accurate predictor of the potential of parental lines for breeding programs aiming to improve traits controlled by metabolic phenotype such as resistance to pests or taste. The complementation of AFLP fingerprints by metabolic profiles for breeding and conservation purposes in sesame is recommended.

\section{Methods}

\section{Plant material}

Seeds were obtained from Centro Nacional de Investigaciones Agropecuarias (CENIAP) Germplasm Bank, Venezuela (Table 3). Plants were germinated and grown in the greenhouse with a photoperiod of 12 hours dark and 12 hours light at $30^{\circ} \mathrm{C}$. 


\section{AFLP analysis}

DNA was extracted from leaves and AFLP analysis was performed based on the protocol by Voss et al. [2] with minor modifications as previously reported [25, 26], using eight primer combinations (Table 1). AFLP reactions were performed twice for each accession, using restriction enzymes EcoRI and Tru1I (MBI Fermentas, Germany) and compatible primers (see Table 1 and Table 7 in [25]). Primers for pre-amplification were extended by one selective nucleotides ( $\mathrm{C}$ for MseI and A for EcoRI). During selective amplification, fluorescent label (Cy5) was attached to the EcoRI primer. DNA fragments were separated on ALFexpress II DNA analyzer (Amersham Pharmacia Biotech, Uppsala, Sweden). Automatic band recognition and matching was done by using GelCompar II software (Applied Math, Belgium). A threshold value of 5\% relative to the maximum value within each lane was applied and only fragments identified in both replicas (between 94 and 100 $\%$ of all bands recorded) were used for band matching. The results of band matching were encoded as a binary matrix, which was used for all further analysis.

\section{Metabolic profiling}

Seeds originating from five plants per accession were bulked and $1 \mathrm{~g}$ of tissue was frozen with liquid nitrogen, ground in a mortar with a pestle and extracted anaerobically with a mixture of $80 \%$ ethanol (gradient grade, Roth, Germany) and $20 \%$ water for $16 \mathrm{~h}$ with stirring $(100 \mathrm{rpm})$. The liquid phase was filtered through $0.2 \mu \mathrm{m}$ filters and kept at $-20^{\circ} \mathrm{C}$ until HPLC analysis.

For HPLC analysis, $10 \mu \mathrm{l}$ aliquots of extracts were loaded onto a polar-modified RP-18 phase column (C18-Pyramid, Macherey-Nagel, Düren, Germany, $3 \mu \mathrm{m}, 2$ x $125 \mathrm{~mm}$ ) and separated at $40^{\circ} \mathrm{C}$ with a gradient of $10 \%-98 \%$ methanol at a flow rate of $0.2 \mathrm{ml}$ min-1. The eluent was subjected to electrospray ionisation (ESI). Ions were analyzed in both positive and negative full scan mode between $\mathrm{m} / \mathrm{z} 50$ and 1000 with an ion trap.

\section{Data processing and analysis}

Raw data from the metabolic study were processed with the CODA algorithm (background reduction and spike elimination [33]). Extracted ion chromatograms with a mass quality index of at least 0.85 (according to technical manual of ACD/MS Manager v. 8.0, 
Advanced Chemistry Development, Toronto, Canada) were generated and compared. Based on these chromatograms, peak tables were generated. Ten peaks with the highest MCQ value for each accession were selected. For each peak, matching peaks in all accessions were identified, building a set of peaks for use in further analysis. Isotope peaks, recognized by the difference of one unit in the molecular weight and the same retention time, were combined to generate one value per metabolite per accession.

Peak areas were standardized twice, first within every accession by dividing the area by the total sum of areas of all peaks for each accession to compensate for loading differences, and second within every $\mathrm{m} / \mathrm{z}$ value (across accessions) by dividing peak areas by the maximum area within the $\mathrm{m} / \mathrm{z}$ value. The purpose of the second normalization was to weight major peaks in each extracted ion chromatogram equally for statistical evaluation, because the relationship between the amount of a substance that enters the ion source and the magnitude of the signal recorded by a mass detector varies among metabolites. Due to the lack of a suitable criterion, no data pretreatment was applied [34]. The resulting matrix was used to calculate correlation coefficients as a measure of similarity between pairs of accessions. To assess the effect of differences in signal intensities within extracted ion chromatograms, the matrix of doubly-normalized intensities was transformed into a binary matrix by replacing all nonzero values with 1 . Using the binary matrix, a simple matching coefficient was calculated for each pair of accessions. The correlation between the correlation coefficient-based matrix and simple matching coefficient-based matrix was calculated by Mantel test (500 permutations). To visualize the relationship among accessions according to their metabolite content, principal component analysis was conducted with the correlation matrix. Principal coordinate analysis was used for the simple matching coefficient matrix. Calculations of similarity and dissimilarity coefficients, principal component and coordinate analysis were performed with NTSySpc 2.11T (Applied Biostatistics, Setauket (NY), USA).

A binary matrix from the AFLP data was obtained and a Jaccard's coefficient similarity matrix was calculated. The relationship among accessions was visualised as a principal coordinate analysis. Comparison of ordination obtained by AFLP and metabolite content was based on Pearson's correlation and a Mantel test between the matrices with 1000 permutations. The two approaches also were compared by scatter plots to visualize the correlation. The variability range in the scatter plots was split into three sections (high similarity, intermediate similarity and low similarity) on both the $\mathrm{X}$ axis and the $\mathrm{Y}$ axis. 
Pairwise comparisons for the same category in both approaches were identified i.e. pairs of accessions that were highly similar both in AFLP and metabolic data, pairs that possessed an intermediate similarity in both data sets, and pairs dissimilar both in genome and metabolome. The results of principal coordinate analysis performed on AFLP data and principal component analysis performed on metabolic data were compared visually.

\section{Authors' contributions}

HL participated in the design of the study, conducted the genomic diversity study and statistical analysis, and drafted the manuscript. AR generated and analyzed metabolic profiles. PK conceived the study, participated in the experimental design and data analysis and wrote parts of the manuscript. All authors read and approved the final version of the manuscript.

\section{Acknowledgements}

This work was supported by the Programme Alban, European Union Programme of High Level Scholarships for Latin America, International $\mathrm{PhD}$ program for Agricultural Sciences in Göttingen University (IPAG) and Universidad Centroccidental Lisandro Alvarado.

\section{References}

1. Ovesná J, Poláková K, Leisová L: DNA analyses and their applications in plant breeding. Czech J Genet Plant Breed 2002, 38:29-40.

2. Voss P, Hogers R, Bleeter M, Reijans M, van de Lee T, Hornes M, Frijters A, Pot J, Peleman J, Kuiper M, Zabeau M: AFLP: a new technique for DNA fingerprinting. Nucleic Acids Res 1995, 23:4407-4414.

3. Li YC, Korol AB, Fahima T, Beiles A, Nevo E: Microsatellites: Genomic distribution, putative functions and mutational mechanisms. Molecular Ecology 2002, 11:2453-65.

4. Dunn W, Ellis D: Metabolomics: Current analytical platforms and methodologies. Trends in Analytical Chemistry 2005, 24:285-294. 
5. Hall RD: Plant metabolomics: From holistic hope, to hype, to hot topic. New Phytologist 2006, 169:453-468.

6. Fiehn O: Metabolomics - the link between genotypes and phenotypes. Plant Molecular Biology 2002, 48:155-171.

7. Fiehn O, Weckwerth W: Deciphering metabolic networks. Eur J Biochem 2003, 270:579-588.

8. Bedigian D and Harlan $\mathrm{J}$ : Evidence for cultivation on sesame in the ancient world. Economic Botany 1986, 40:137-154.

9. Bedigian D: Evolution of sesame revisited: domestication, diversity and prospects. Genet Res Crop Evol 2003, 50: 779-787.

10. Yoshida H, Takagi S: Effects of seed roasting temperature and time on the quality characteristics of sesame (Sesamum indicum) oil. J Sci Food Agric 1997, 75:19-26.

11. Shyu Y, Hwang L: Antioxidative activity of the crude extract of lignan glycosides from unroasted Burma black sesame meal. Food Research International 2002, 35:357-365.

12. Dachtler M, van de Put F, Stijn F, Beindorff C, Fritsche J: On-line LC-NMR-MS characterization of sesame oil extracts and assessment of their antioxidant activity. Eur J Lipid Sci Technol 2003, 105:488-496.

13. Shirato-Yasumoto S, Komeichi M, Okuyama Y, Horigane A: A simplified HPLC quantification of sesamin and sesamolin in sesame seed. SABRAO Journal of Breeding and Genetics 2003, 35:27-34.

14. Kang MH, Naito M, Tsujihara N, Osawa T: Sesamolin inhibits lipid peroxidation in rat liver and kidney. $J$ Nutr 1998, 128:1018-1022.

15. Kang MH, Kawai Y, Naito M, Osawa T: Dietary defatted sesame flour decreases susceptibility to oxidative stress in hypercholesterolemic rabbits. $J$ Nutr 1999 , 129:1885-1890.

16. Kang MH, Naito M, Sakai K, Uchida K, Osawa T: Mode of action of sesame lignans in protecting low-density lipoprotein against oxidative damage in vitro. Life Sci 2000, 66:161-171.

17. Miyake Y, Fukumoto S, Okada M, Sakaida K, Nakamura Y, Osawa T: Antioxidative catechol lignans converted from sesamin and sesaminol triglucoside by culturing with Aspergillus. J Agric Food Chem 2005, 53:22-27. 
18. Devine G, Denholm I: An unconventional use of piperonyl butoxide for managing the cotton whitefly, Bemisia tabaci (Hemiptera:Aleyrodidae). Bulletin of Entomological Research 1988, 88:601-610.

19. Devine G, Ishaaya I, Horowitz A, Denholm I: The response of pyriproxyfenresistant and susceptible Bemisia tabaci Genn. (Homoptera:Aleyrodidae) to pyriproxyfen and fenoxycarb alone and in combination with piperonyl butoxide. Pestic Sci 1999, 55:405-411.

20. Brooker N, Long J, Stephan S: Field assessment of plant derivative compound for managing fungal soybean diseases. Biochemical Society Transactions 2000, 28:917-920.

21. Victor S, Crisóstomo F, Bueno F, Pagnocca F, Fernandes J, Correa A, Bueno O, Hebling J, Bacci Jr. M, Vieira P, daSilva F: Toxicity of synthetic piperonyl compounds to leaf-cutting ants and their symbiotic fungus. Pest Manag Sci 2001, 57:603-608.

22. Harmatha J, Nawrot $\mathrm{J}$ : Insect feeding deterrent activity of lignans and related phenylpropanoids with a methylenedioxyphenyl (pyperonyl) structure moiety. Entomologia Experimentalis et Applicata 2002, 104:51-60.

23. Nascimento I, Murata A, Bortoli S, Lopes L: Insecticidad activity of chemical constituents from Aristolochia pubescens against Anticarsia gemmatalis larvae. Pest Manag Sci 2004, 60:413-416.

24. Peñalvo J, Heinonen S, Aura A, Adlerreutz H: Dietary sesamin in converted to enterolactone in humans. $J$ Nutr 2005, 135:1056-1062.

25. Laurentin H, Karlovsky $P$ : Genetic relationship and diversity in a sesame (Sesamum indicum L.) germplasm collection using amplified fragment length polymorphism (AFLP). BMC Genetics 2006, 7:10.

26. Laurentin H, Karlovsky P: AFLP fingerprinting of sesame (Sesamum indicum L.) cultivars: identification, genetic relationship and comparison of AFLP informativeness parameters. Genetic Resources and Crop Evolution 2007, 54:1437-1446.

27. Wolde-meskel E, Terefework Z, Lindstrom K, Frostegard A: Metabolic and genomic diversity of rhizobia isolated from field standing native and exotic woody legumes in southern Ethiopia. Syst Appl Microbiol 2004, 27:603-611.

28. Seymour F, Cresswell J, Jack P, Lappin-Scott H, Haag H, Talbot N: The influence 
of genotypic variation on metabolite diversity in populations of two endophytic fungal species. Fungal Genetics and Biology 2004, 41:721-734.

29. Keurentjes JJ, Fu J, de Vos CH, Lommen A, Hall RD, Bino RJ, van der Plas LH, Jansen RC, Vreugdenhil D, Koornneef M: The genetics of plant metabolism. Natural Genetics 2006, 38:842-849.

30. De Vos RC, Moco S, Lommen A, Keurentjes JJ, Bino RJ, Hall RD: Untargeted large-scale plant metabolomics using liquid chromatography coupled to mass spectrometry. Nature Protocols 2007, 2:778-791.

31. Dixon RA, Gang DR, Charlton AJ, Fiehn O, Kuiper HA, Reynolds TL, Tjeerdema RS, Jeffery EH, German JB, Ridley WP, Seiber JN: Applications of metabolomics in agriculture. J Agric Food Chem 2006, 54:8984-8994.

32. Rao N: Plant genetic resources: advancing conservation and use through biotechnology. African Journal of Biotechnology 2004, 3:136-145.

33. Windig W, Phalp JM, Payne AW: Noise and Background Reduction Method for Component Detection in Liquid Chromatography/Mass Spectrometry. Anal Chem 1996, 68:3602-3606.

34. Berg RA van den, Hoefsloot HCJ, Westerhuis JA, Smilde AK, Werf MJ van der: Centering, scaling, and transformations: improving the biological information content of metabolomics data. BMC Genomics 2006, 7:142.

35. Joy N, Abraham Z, Soniya EV: A preliminary assessment of genetic relationships among agronomically important cultivars of black pepper. $B M C$ Genetics 2007, 8:42.

36. Bhattacharya E, Ranade SA: Molecular distinction amongst varieties of mulberry using RAPD and DAMD profiles. BMC Plant Biology 2001, 1:3.

37. Awasthi AK, Nagaraja GM, Naik GV, Kanginakudru S, Thangavelu K, Nagaraju J: Genetic diversity and relationships in mulberry (genus Morus) as revealed by RAPD and ISSR marker assays. BMC Genetics 2004, 5:1.

38. Dangi RS, Lagu MD, Choudhary LB, Ranjekar PK, Gupta VS: Assessment of genetic diversity in Trigonella foenum-graecum and Trigonella caerulea using ISSR and RAPD markers. BMC Plant Biol 2004, 4:13.

39. Bhattacharya E, Dandin SB, Ranade SA: Single primer amplification reaction methods reveal exotic and indigenous mulberry varieties are similarly diverse. J Biosci 2005, 30:669-77. 
40. Tam SM, Mhiri C, Vogelaar A, Kerkveld M, Pearce SR, Grandbastien MA: Comparative analyses of genetic diversities within tomato and pepper collections detected by retrotransposon-based SSAP, AFLP and SSR. Theor Appl Genet 2005, 110:819-31.

41. Karlovsky, P: Secondary Metabolites in Soil Ecology. In Soil Biology. Volume 2. Edited by P. Karlovsky (Editor in Chief A. Varma). New York: Springer; 2007:119.

42. Willför SM, Smeds AI, Holmbom BR: Chromatographic analysis of lignans. $J$ Chromatography A 2006, 1112:64-77.

43. Dachtler M, van de Put FHM, Stijn Fv, Beindorff CM, Fritsche J: On-line LCNMR-MS characterization of sesame oil extracts and assessment of their antioxidant activity. Eur J Lipid Sci Technol 2003, 105:488-96.

44. Lim JS, Adachi Y, Takahashi Y, Ide T: Comparative analysis of sesame lignans (sesamin and sesamolin) in affecting hepatic fatty acid metabolism in rats. $\mathrm{Br} J$ Nutr 2007, 97:85-95.

45. Yasumoto SS, Komeichi M, Okuyama Y, Horigane A: A simplified HPLC quantification of sesamin and sesamolin in sesame seed. SABRAO J Breed Gen 2003, 35:27-34.

46. Williamson KS, Morris JB, Pye QN, Kamat CD, Hensley K: A survey of sesamin and composition of tocopherol variability from seeds of eleven diverse sesame (Sesamum indicum L.) genotypes using HPLC-PAD-ECD. Phytochem Anal. 2007 [Epub ahead of print], DOI: 10.1002/pca.1050

47. Hettwer U, Laurentin H, Karlovsky P: Determination of antioxidative furofuran lignans in sesame seeds by HPLC-MS. In Proceedings of the Second International Symposium on Recent Advances in Food Analysis, Last Minute Posters: 2-4 November 2005; Prague. Edited by Hajslova J and Nielen MWF. Abingdon: Taylor and Francis; 2006:15.

48. Laurentin H, Layrisse A, Quijada P: Evaluación de dos ciclos de selección recurrente para altos rendimientos de semilla en una población de ajonjolí. Agronomía Tropical (Maracay) 2000, 50:521-535.

49. Mazzani B: Inamar: nueva variedad de ajonjolí producida en el Instituto Nacional de Agricultura. Agronomía Tropical (Maracay) 1953, 3:211-213. 


\section{Tables and Figures}

Table 1. AFLP: Primer combinations and polymorphism of DNA bands

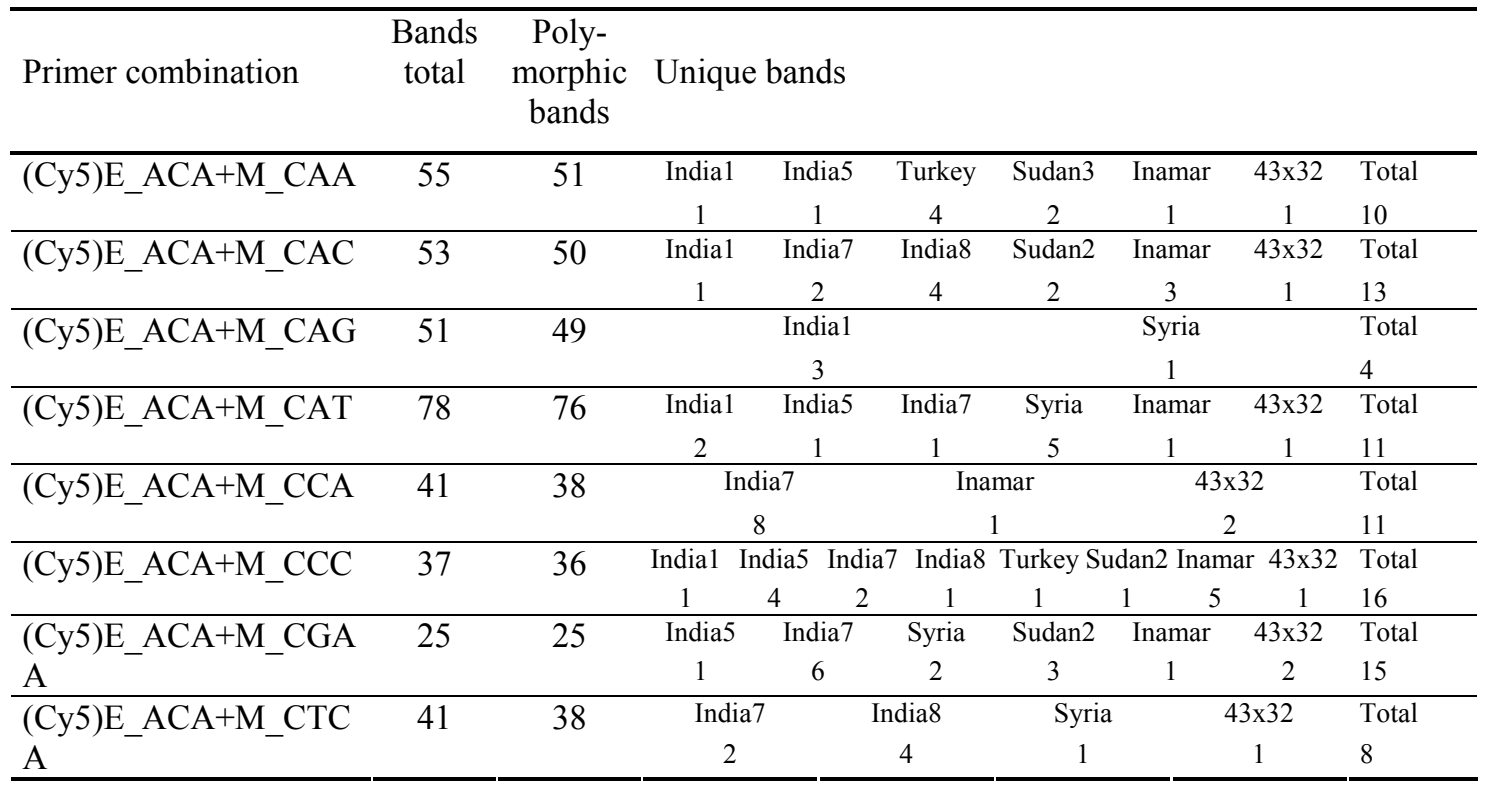


Table 2. Metabolic signals in sesame seed extracts used in the analysis

\begin{tabular}{|c|c|c|c|c|c|}
\hline \multicolumn{6}{|c|}{ Negative mode } \\
\hline Mass range & $\begin{array}{c}\mathrm{m} / \mathrm{z} \\
\text { values } \\
\text { total }\end{array}$ & $\begin{array}{l}m / z \text { values common } \\
\text { to all accessions }\end{array}$ & \multicolumn{3}{|c|}{$\begin{array}{l}\text { Number of accession-specific } m / z \text { values } \\
\text { or values lacking in only one accession }\end{array}$} \\
\hline \multirow[t]{2}{*}{$200-400$} & \multirow[t]{2}{*}{14} & \multirow[t]{2}{*}{3} & \multicolumn{2}{|c|}{ Sudan 2} & Total \\
\hline & & & \multicolumn{2}{|c|}{2} & 2 \\
\hline \multirow[t]{2}{*}{$400-600$} & \multirow[t]{2}{*}{11} & \multirow[t]{2}{*}{1} & \multicolumn{2}{|c|}{ Turkey } & Total \\
\hline & & & \multicolumn{2}{|c|}{1} & 1 \\
\hline \multirow[t]{2}{*}{$600-800$} & \multirow[t]{2}{*}{17} & \multirow[t]{2}{*}{10} & \multicolumn{2}{|c|}{ India 5} & Total \\
\hline & & & \multicolumn{2}{|c|}{1} & 1 \\
\hline \multirow[t]{2}{*}{$800-1000$} & \multirow[t]{2}{*}{5} & \multirow[t]{2}{*}{4} & \multirow{2}{*}{\multicolumn{2}{|c|}{$\frac{\text { India } 5}{1}$}} & Total \\
\hline & & & & & 1 \\
\hline \multicolumn{6}{|c|}{ Positive mode } \\
\hline Mass range & $\begin{array}{c}\mathrm{m} / \mathrm{z} \\
\text { values } \\
\text { total }\end{array}$ & $\begin{array}{l}m / z \text { values common } \\
\text { to all accessions }\end{array}$ & \multicolumn{3}{|c|}{$\begin{array}{l}\text { Number of accession-specific } m / z \text { values } \\
\text { or values lacking in only one accession }\end{array}$} \\
\hline \multirow{3}{*}{$\frac{400-600}{600-800}$} & 2 & 1 & & & \\
\hline & \multirow[t]{2}{*}{25} & \multirow[t]{2}{*}{6} & India 8 & $43 \times 32$ & Total \\
\hline & & & & 1 & 2 \\
\hline \multirow[t]{2}{*}{$800-1000$} & \multirow[t]{2}{*}{14} & \multirow[t]{2}{*}{9} & \multicolumn{2}{|c|}{ India 5} & Total \\
\hline & & & \multicolumn{2}{|c|}{1} & 1 \\
\hline
\end{tabular}


Table 3. Sesame accessions

\begin{tabular}{|l|c|c|c|}
\hline \multicolumn{5}{|c|}{ CENIAP Germplasm Bank } \\
\hline Accessions & Country of Origin & Working code & Diversity Centre \\
\hline $93-2223$ & India & India 1 & India \\
\hline $89-007$ & India & India 5 & India \\
\hline $95-464$ & India & India 7 & India \\
\hline $92-2918$ & India & India 8 & India \\
\hline $92-2922$ & Turkey & Turkey & Western Asia \\
\hline $93-2022$ & Syria & Syria & Western Asia \\
\hline $92-310$ & Sudan & Sudan 2 & Africa \\
\hline $92-2872$ & Sudan & Sudan 3 & Africa \\
\hline \multicolumn{5}{|c|}{$\begin{array}{c}\text { Venezuelan accessions } \\
\text { Accessions }\end{array}$} & $\begin{array}{c}\text { Country of Origin } \\
\text { 43x32 }\end{array}$ & $\begin{array}{c}\text { Generated in } \\
\text { Venezuela }\end{array}$ & $\begin{array}{l}\text { Line selected from a second cycle of } \\
\text { recurrent selection toward high yield } \\
\text { under heavy whitefly infestation. The } \\
\text { original population was obtained by } \\
\text { cross, one to one, among 50 exotic } \\
\text { accessions [48] }\end{array}$ \\
\hline $\begin{array}{c}\text { Experimental line } \\
\text { Commercial cultivar } \\
\text { "Inamar" }\end{array}$ & \multicolumn{2}{|c|}{$\begin{array}{c}\text { Developed in } \\
\text { Venezuela }\end{array}$} & $\begin{array}{l}\text { Individual selection from the offspring } \\
\text { from the same Acarigua's parents [49] }\end{array}$ \\
\hline
\end{tabular}



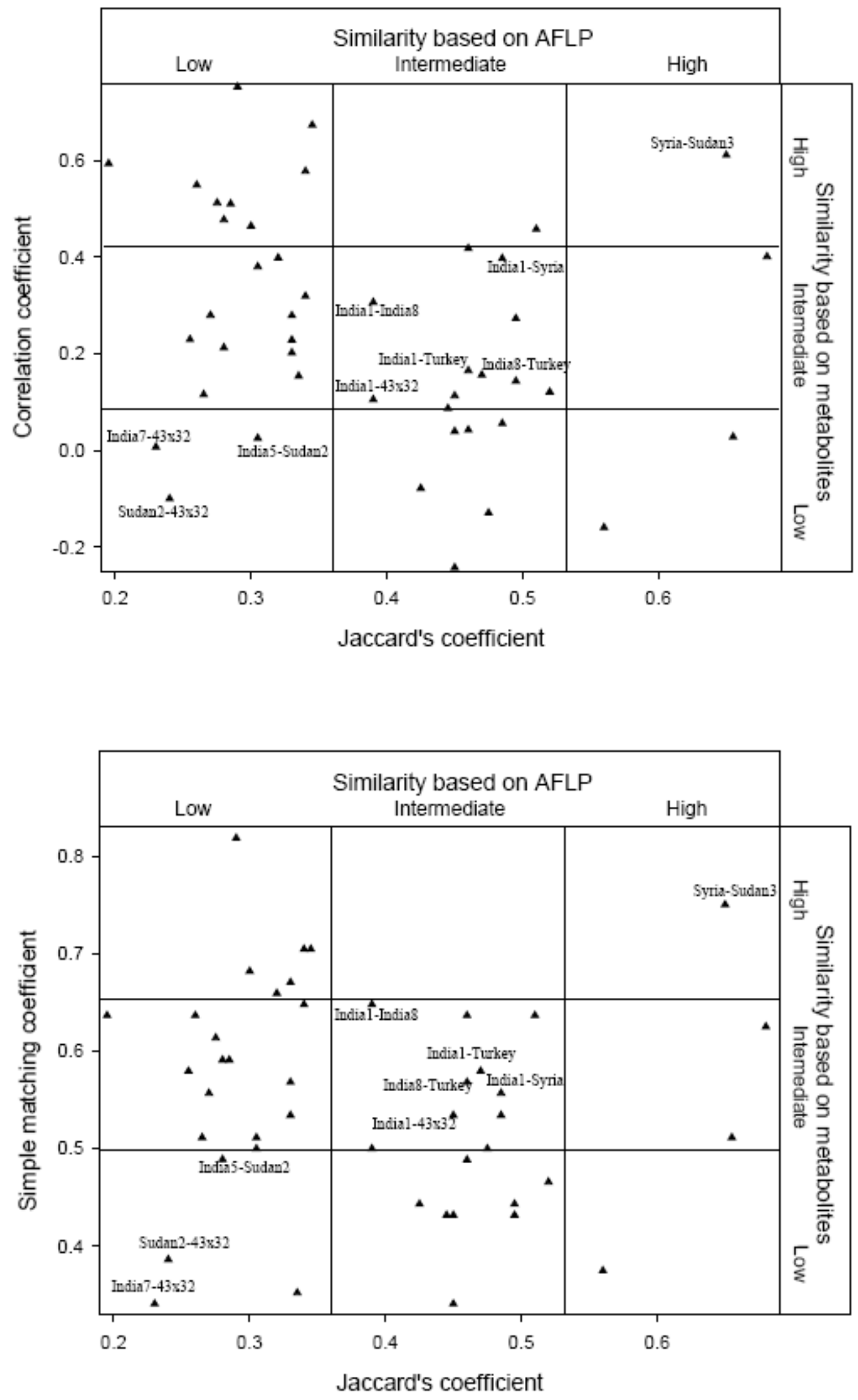

Figure 1. Scatter plots comparing ordination based on AFLP (Jaccard's coefficient) with ordination based on metabolic profiles 
Upper part: Metabolic profile comparisons based on quantitative variables (correlation coefficient). Lower part: Metabolic profile comparisons based on binary variables (simple matching coefficient). Accessions in pairwise comparisons which have a high, intermediate or low similarity for both approaches are labeled.

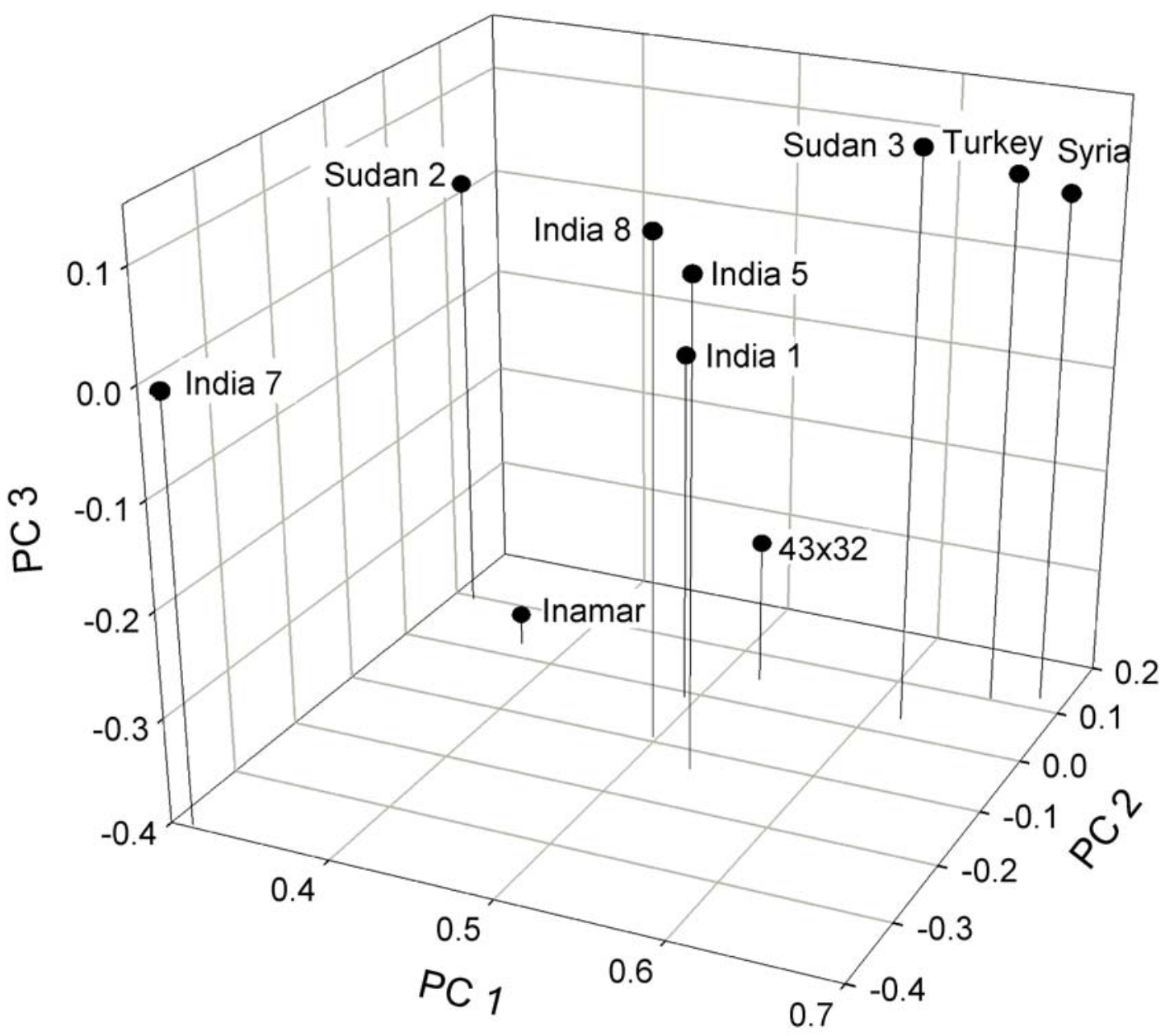

Figure 2. Biplot of principal coordinate analysis based on Jaccard's coefficient for AFLP 


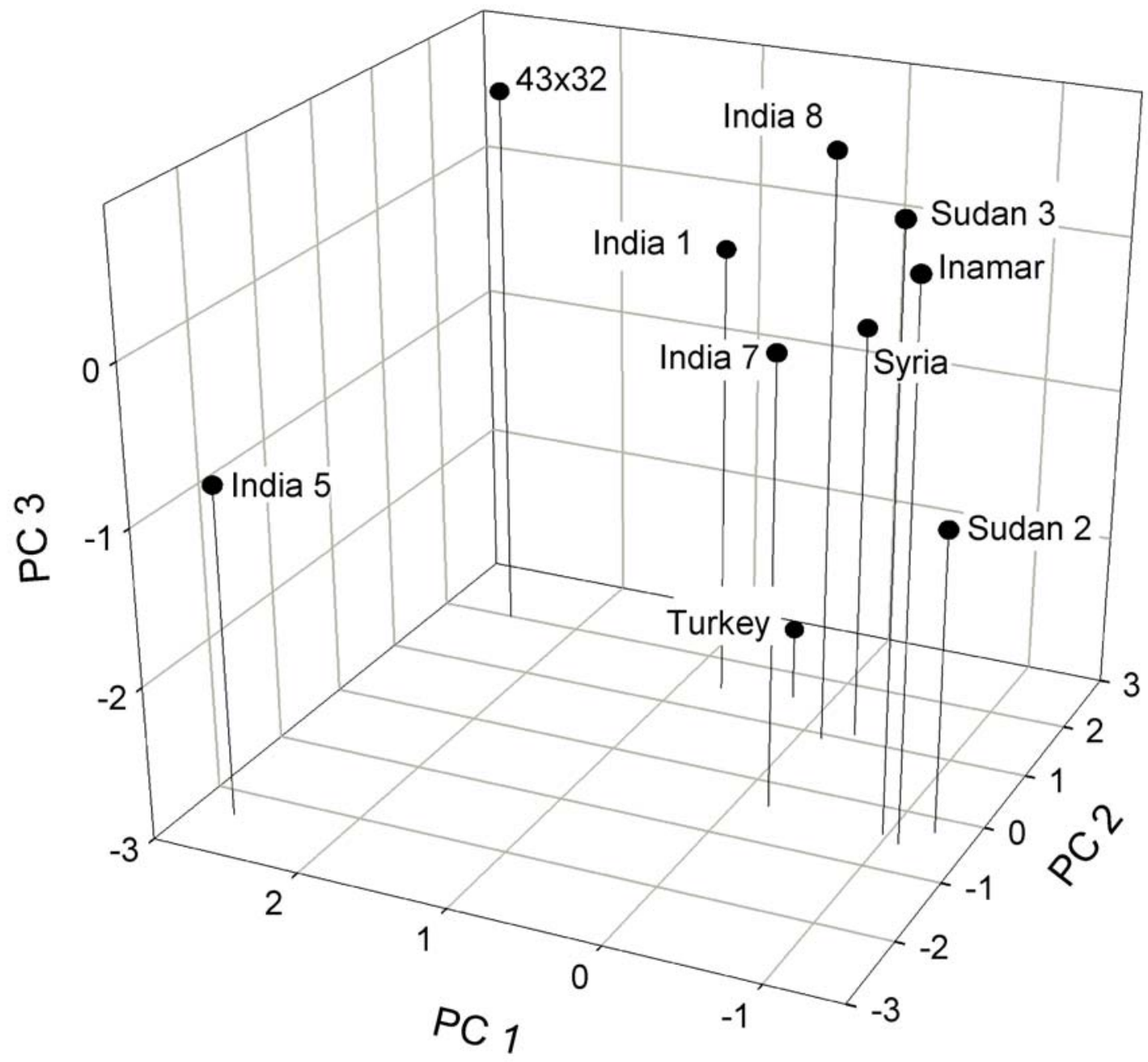

Figure 3. Biplot of principal components analysis based on correlation coefficient for seed metabolic profiles 


\title{
Chapter 5: Identification of soil $P$ mobilizing components of sugar beet root exudates after differential metabolic profiling by HPLC-MS*
}

*This manuscript has been submitted for publication.

\author{
Reza Khorassani ${ }^{1,2}$, Ursula Hettwer ${ }^{3}$, Astrid Ratzinger ${ }^{3}$, Bernd Steingrobe ${ }^{1}$, \\ Petr Karlovsky ${ }^{3}$ Norbert Claassen ${ }^{1}$
}

1- Department of Crop Science-Plant nutrition, Georg-August-University Göttingen, Carl-Sprengel-Weg 1, D-37075 Göttingen, Germany

2- Department of Soil Science, Faculty of Agriculture, Ferdowsi University of Mashhad, Iran

3- Department of Crop Science-Molecular Phytopathology and Mycotoxin Research, Georg-August-University Göttingen, Grisebachstrasse 6, D-37077 Göttingen, Germany 


\section{Abstract}

At low P supply of the soil sugar beet has shown to have a much higher P influx than other species like maize, wheat or groundnut. It is postulated that this is because sugar beet roots exude organic compounds into the rhizosphere capable to mobilize, i.e., solubilize, soil P. Some organic acids like citric and oxalic acid have been studied as P mobilizing agents before but others may also play a role. The objective of this study was to identify compounds secreted in enhanced amounts under conditions of $\mathrm{P}$ deficiency as compared to sufficient P level and assess their ability to mobilize soil P. Root exudates of sugar beet were collected under low and high $\mathrm{P}$ treatments in hydroponic culture. Freeze dried samples were analyzed by HPLC and root exudate components were detected by mass spectrometry (HPLC-MS) in the range of mass-to-charge ratio $(\mathrm{m} / \mathrm{z})$ from 50 to 1000 in both positive and negative ionization modes. 65 signals, enhanced at least 5-times in low $\mathrm{P}$ treatment as compared to high P treatment, were detected. Signals with $\mathrm{m} / \mathrm{z} 137$ and 147 (negative ionization mode) were selected for further analysis. Compounds with the corresponding molecular weights were retrieved from KEGG database and tested for their ability to mobilize soil $\mathrm{P}$ by an incubation experiment. Phosphorus mobilization was characterized by the increase of $\mathrm{P}$ in solution of a soil suspension with $1 \mathrm{mmol} \mathrm{L}^{-1}$ of the compounds studied in comparison to a soil suspension in water. Salicylic acid of the $m / z$ 137 and citramalic acid of the $\mathrm{m} / \mathrm{z} 147$ showed the highest efficiency to mobilize soil $\mathrm{P}$. The enhancement of further HPLC-MS signals under low P conditions indicates that further components of sugar beet root exudates different from citramalic and salicylic acid might participate in the P mobilization in soil.

Key words: Root exudates, Phosphorus mobilization, HPLC-MS technique, Sugar beet, Salicylic acid, Citramalic acid 


\section{Introduction}

Sugar beet and wheat are similar in their $\mathrm{P}$ efficiency with regard to shoot production (Bhadoria et al., 2002). However, they use different P efficiency mechanisms. Wheat has a comparably large root system which compensates for low P influx at low P availability whereas sugar beet is able to achieve a high $\mathrm{P}$ influx despite a low $\mathrm{P}$ soil concentration (Bhadoria et al., 2002). These findings for sugar beet were confirmed in a preliminary experiment with respect to maize and groundnut (unpublished). The higher $\mathrm{P}$ influx of sugar beet compared to other species cannot solely be due to a more efficient uptake physiology. At low $\mathrm{P}$ availability, soil $\mathrm{P}$ transport is the limiting factor in $\mathrm{P}$ uptake (Kovar and Claassen, 2005). Hence, the high $P$ influx of sugar beet is attributed to the ability of the plant to mobilize $\mathrm{P}$ in the soil. This mobilization is most likely due to chemical modification of the rhizosphere by root exudates.

Plants are exuding a relatively high amount of organic compounds into the rhizosphere. According to the review of Lynch and Whipps (1990) $5-30 \%$ of assimilated carbon is released into the soil. The composition of root exudates is complex; they comprise high (HMW) and low molecular weight (LMW) molecules (Marschner, 1995). High molecular weight exudates are e.g. ectoenzymes and the mucilage consisting mainly of polysaccharides. Liebersbach et al. (2004) could show that HMW exudates can increase P availability for plants, probably because carboxyl groups of the polysaccharides interact with $\mathrm{P}$ binding places in the soil which releases $\mathrm{P}$ to soil solution. The long chains of polygalacturonate may also cover soil particles and reduce the re-adsorption of phosphate (Grimal et al., 2001). Furthermore, the ability of HMW exudates to swell and keep water may facilitate $\mathrm{P}$ diffusion towards the root. Low molecular weight exudates include organic acids, sugars, phenolics, amino acids, phytosiderophores, flavonoids and vitamins (Marschner, 1995; Whipps, 1990). Phenolics might affect the speciation of iron by complexation and by this might increase the availability of $\mathrm{P}$ occluded by Fe-oxides (Marschner and Römheld, 1994). Organic acids, especially citrate, malate and oxalate, are root exudates most frequently investigated with regard to $\mathrm{P}$ mobilization. Phosphorus deficiency usually increases the exudation rate and alters the composition of exudates 
(Liebersbach et al., 2004; Bertin et al., 2003) which results in an enhanced release of organic acids into the soil (Johnson et al., 1996; Neumann and Römheld, 1999). Hernandez (2007) showed that in common bean the amount of organic acids in P-stressed roots were decreased as compared to P-sufficient roots; the reduced amount of organic acids in Pdeficient roots more than likely reflects exudation from the root into the rhizosphere. These observations indicate that organic acids are involved in $\mathrm{P}$ acquisition. Different mechanisms have been proposed for the facilitation of $\mathrm{P}$ acquisition by organic acids. For instance, citrate might increase availability of $\mathrm{P}$ in the soil by binding calcium and thus reducing the availability of calcium for the formation of insoluble complexes with $\mathrm{P}$ (Dinkelaker et al., 1989). Citrate may also replace phosphate from $\mathrm{Fe}$ - and Aloxides/hydroxides (ligand exchange). These processes release $\mathrm{P}$ into the soil solution as shown by Gerke et al. (2000) who described a positive relation between citrate sorption and $\mathrm{P}$ solution concentration. Furthermore, citrate may complex iron, forming a citrateiron-P polymer which is soluble and can diffuse to the roots. The root reduces the iron which breaks the polymer and releases P directly at the root surface (Gardener et al., 1983). Hence, in soil incubation experiments with different organic acids, citrate often showed the largest effect on P release.

However, it is still not clear whether the beneficial effect of citrate and other organic acids on $\mathrm{P}$ availability is relevant in the rhizosphere. Most soil incubation experiments investigating the P-releasing effect of citrate were performed at relatively high concentrations $(>1 \mathrm{mM})$ of citrate but the amount of organic acids released by roots is relatively low. Furthermore, organic acids are rapidly decomposed by rhizosphere microorganisms and adsorbed to soil particles. Because the concentration of citrate in the rhizosphere is very low (typically $<10 \mu \mathrm{M}$; Jones, 1998), the contribution of citrate to $\mathrm{P}$ release remains questionable. An exception is the rhizosphere of cluster roots where root surface area and exudation rate are extremely high as compared to non-clustered roots and the microbial activity is reduced due to a low pH value (Hoffland et al., 1989; Kania et al., 2001). Under these conditions, citrate might be a key factor for increasing the availability of P for plants.

For plants without cluster roots, i.e. by far most plant species, other root exudate compounds - besides or together with citrate - may play an important role in increasing $\mathrm{P}$ 
availability. For example, the high $\mathrm{P}$ uptake efficiency of pigeon pea is due to the exudation of piscidic acid (Ae et al., 1990), which is exuded in lower amounts than citrate but releases P more efficiently. So far, only pigeon pea is known to rely on piscidic acid, which indicates differences between plant species in the ability to increase P availability by root exudation.

The objective of this study was to identify compounds in root exudates of sugar beet which might increase $\mathrm{P}$ availability in the soil and, thus, be responsible for the high $\mathrm{P}$ uptake efficiency of sugar beet. The exudates were collected at three plant ages from plants grown at sufficient $\mathrm{P}$ supply and under $\mathrm{P}$ deficiency in solution culture. Compounds of root exudates were identified by differential metabolic profiling using the HPLC-MS and tested for their ability to solubilize $\mathrm{P}$ in soil.

\section{Materials and methods}

\section{Hydroponic experiment}

Sugar beet plants were grown in hydroponic culture in a growth chamber. Sugar beet seeds were sown in sand and grown only with distilled water for 14 days. Seedlings of similar size were selected and washed free of sand by distilled water and carefully transferred into pots containing $12 \mathrm{~L}$ of aerated nutrient solution. The experiment was carried out with two treatments of $\mathrm{P}$, three harvests and three replications in a growth chamber regulated at 16/8 h light/dark cycle, $25{ }^{\circ} \mathrm{C} / 16{ }^{\circ} \mathrm{C}$ day/night temperature, $60 \% / 75 \%$ relative humidity and 41 $\mathrm{W} \mathrm{m} \mathrm{m}^{-2} \mathrm{PAR}$ (photosynthetic active radiation during the day time). The composition of the nutrient solution was $1 \mathrm{mM} \mathrm{Ca}\left(\mathrm{NO}_{3}\right)_{2} .4 \mathrm{H}_{2} \mathrm{O}, 0.1 \mathrm{mM} \mathrm{NH} \mathrm{NO}_{3}, 0.2 \mathrm{mM} \mathrm{KCl}, 0.1 \mathrm{mM}$ $\mathrm{MgSO}_{4} .7 \mathrm{H}_{2} \mathrm{O}, 17.9 \mu \mathrm{M}$ Fe-EDTA, $16 \mu \mathrm{M} \mathrm{H} \mathrm{H}_{3} \mathrm{BO}_{3}, 9.1 \mu \mathrm{M} \mathrm{MnCl}_{2} .4 \mathrm{H}_{2} \mathrm{O}, 0.8 \mu \mathrm{M}$ $\mathrm{ZnSO}_{4} .7 \mathrm{H}_{2} \mathrm{O}, 0.5 \mu \mathrm{M}\left(\mathrm{NH}_{4}\right)_{6} \mathrm{Mo}_{7} \mathrm{O}_{24} .4 \mathrm{H}_{2} \mathrm{O}, 0.3 \mu \mathrm{M} \mathrm{CuSO}_{4} .5 \mathrm{H}_{2} \mathrm{O}$. The P concentrations in nutrient solution were 2 or $500 \mu \mathrm{M} \mathrm{P}$ as $\mathrm{NaH}_{2} \mathrm{PO}_{4}$. To simplify the terminology, 2 and 500 $\mu \mathrm{M} \mathrm{P}$ were designated as low-P and high-P. Fifteen, ten and six plants were placed in pots for the first, second and third harvest, respectively. Plants were harvested every two weeks after transplanting. Replacement of the nutrient solution and $\mathrm{pH}$ measurements were conducted every second day. The root exudates were collected at each harvest. 


\section{Root exudates sampling}

Whole root systems of intact plants were carefully washed with running de-ionized water to remove the nutrient solution. For collection of exudates, the whole root system was dipped into aerated de-ionized water in glass container with appropriate volume for different sizes of the root systems depending on plant age. The container was covered with aluminum foil to create dark conditions for roots. The collection experiment was carried out for two hours in the same controlled climate conditions as where plants were grown. For the short exudation period of two hours it is unlikely that root membranes were harmed by the de-ionized water and significant microbial degradation of the exudates took place (Neumann and Römheld, 2001). Collected root exudates were immediately frozen at $-30^{\circ} \mathrm{C}$, freeze dried and the dry matter weight recorded.

\section{Preparation of samples and standards for HPLC-MS analysis}

$0.5 \mathrm{mg}$ of freeze-dried root exudates were weighed into an HPLC vial and $100 \mu \mathrm{L}$ of acetonitrile were added. After half an hour, samples were vigorously shaken. Then $900 \mu \mathrm{L}$ of HPLC-quality water were added, the samples were shaken again and filtered through Teflon membrane filters $(0.2 \mu \mathrm{m}$ Optiflow-TF with $13 \mathrm{~mm}$ diameter, Wicom Germany $\mathrm{GmbH}$, Heppenheim, Germany). Pooled root exudates were prepared as a mixture of $100 \mu \mathrm{L}$ of each single sample.

For identification experiments citramalic acid and salicylic acid were prepared as $10 \mathrm{mg}$ $\mathrm{mL}^{-1}$ stock solutions in methanol:water $(50: 50 \mathrm{v} / \mathrm{v})$. Specific transitions were evaluated for $10 \mu \mathrm{g} \mathrm{mL}^{-1}$ solutions in methanol:water $(50: 50 \mathrm{v} / \mathrm{v})$ whereas for the investigation of chromatographic behavior stock solutions were diluted to $100 \mathrm{ng} \mathrm{mL}^{-1}$ in the respective column equilibration buffer.

\section{HPLC-MS instruments and analysis}

\section{Metabolic profiling of root exudates}

For metabolic profiling of root exudates, an HPLC system equipped with an ion trap mass spectrometer was used. The system consisted of ProStar 410 Autosampler, the ProStar 210 solvent delivery module (both Varian Deutschland GmbH, Darmstadt, Germany), and the column oven (TECHLAB GmbH, Erkerode, Germany). A Polaris C18-Ether column (100 
x $2 \mathrm{~mm}$ i.d., $3 \mu \mathrm{m}$ particle size) and a guard column MetaGuard (both Varian) were used. Mass spectrometry was performed with the 500-MS ion trap connected to an electrospray ion source (Varian). Samples were eluted with a gradient of (A) bi-distilled water:acetonitrile (95:5) and (B) methanol as follows: 0-2.2 min: 10\% B; 2.2-25.0 min: 10$98 \% \mathrm{~B} ; 25.0$ to $30.0 \mathrm{~min}: 98 \%$ B. Separation took place at $40^{\circ} \mathrm{C}$ at a flow rate of $0.2 \mathrm{~mL}$ $\min ^{-1}$. Nebulizing (-ESI air/ +ESI nitrogen) and drying gas (nitrogen) pressures were set to 50 and $20 \mathrm{psi}\left(345\right.$ and $138 \mathrm{kPa}$ ), respectively. Drying gas temperature was set to $350^{\circ} \mathrm{C}$ at the beginning of the gradient and reduced gradually to $250^{\circ} \mathrm{C}$ with the increasing proportion of methanol in the eluate. For the detection of positive and negative ions, needle voltages were set to 5000 and $-3500 \mathrm{~V}$ and shield voltage to 600 and $-600 \mathrm{~V}$, respectively. The capillary voltage was $+/-50 \mathrm{~V}$. In positive ionization mode, ions with $\mathrm{m} / \mathrm{z} 100$ to 1000 were collected in a single run, while in negative mode ranges 50-400 and 400-1000 were scanned separately. The scan rate was $5000 \mathrm{Da} / \mathrm{s}$ and three consecutive scans were averaged. MS data were transformed into chromatograms using MS Data Review 6.9 (Varian) and converted into netCDF format.

\section{Targeted analysis and identity verification for selected metabolites}

HPLC-MS-MS detection of specific compounds was performed on an identical HPLC system with a triple quadrupole mass spectrometer (1200 L, Varian). Chromatography was performed on a polar-modified RP-18 phase and on a HILIC (Hydrophilic interaction chromatography) phase. Root exudates and test substances were separated under identical conditions. For RP-18 chromatography a buffer system of (A) water with 5\% acetonitrile and (B) methanol was used. $10 \mu \mathrm{L}$ of sample were loaded to a Synergi Fusion RP column (50 x 2 mm i.d., $2 \mu \mathrm{m}$ particle size; Phenomenex, Aschaffenburg, Germany) equilibrated with $10 \% \mathrm{~B}$ and separated in a linear gradient of $10-30 \% \mathrm{~B}$ in $10 \mathrm{~min}$. For HILIC the buffer system consisted of (A) $25 \mathrm{mM}$ ammonium acetate and (B) acetonitrile with $25 \mathrm{mM}$ ammonium acetate. $10 \mu \mathrm{L}$ of sample were loaded to a ZIC-HILIC (Sequant, Haltern am See, Germany) equilibrated with $95 \% \mathrm{~B}$ and separated in a linear gradient from $95-10 \% \mathrm{~B}$ in $10 \mathrm{~min}$. For both systems, the columns were kept at $40^{\circ} \mathrm{C}$ and the flow rate was $0.2 \mathrm{~mL}$ $\min ^{-1}$. The RP-18 and HILIC columns were washed and re-equilibrated for 5 and $20 \mathrm{~min}$, respectively. Salicylic acid was identified by the mass transition $137>93\left[\mathrm{M}-\mathrm{H}-\mathrm{CO}_{2}\right]^{-}$and a retention time of $7.15 \mathrm{~min}$ on the polar modified RP-18 phase. Citramalic acid was 
identified by the mass transition $147>87\left[\mathrm{M}-\mathrm{H}-\mathrm{CH}_{3} \mathrm{COOH}\right]^{-}$and a retention time of 9.2 min on the HILIC phase.

\section{HPLC-MS data analysis}

Data from positive and negative ionization modes were separately processed as follows: Minor differences in retention times were corrected by peak alignment performed with XCMS version 1.5.2 (Smith et al., 2006) run under R package 2.4.0. The resulting data were normalized using a Perl script (Karlovsky, unpublished) to compensate for differences caused by uneven preparation of the samples. Briefly, the procedure consisted of the selection of constitutive signals, calculation of normalization factor as a mean of the ratio of intensities under low $\mathrm{P}$ and high $\mathrm{P}$ conditions, and multiplication of signals in the high P chromatogram by the normalization factor.

\section{P solubility experiment}

A highly $\mathrm{P}$ fixing fossile Oxisol, as used in a preliminary experiment on $\mathrm{P}$ efficiency of sugar beet, maize and groundnut, was fertilized with $100 \mathrm{mg} \mathrm{P} \mathrm{kg}^{-1}$ as $\mathrm{Ca}\left(\mathrm{H}_{2} \mathrm{PO}_{4}\right)_{2} \cdot \mathrm{H}_{2} \mathrm{O}$ and moistured to $22 \% \mathrm{w} / \mathrm{w}$ water content. After 10 days of equilibration the effect of 4-hydroxy benzoic acid (Fluka, Germany), urocanic acid (Fluka, Germany) and salicylic acid (Merck, Germany) for mass of 138, and citramalic acid (Aldrich, Germany), D-arabino-1,4-lactone (Dextera, UK) and Pantolactone (Aldrich, Germany) (Pantonic acid was generated from the respective lactone by dissolving the lactone in $0.1 \mathrm{M} \mathrm{NaOH}$ ) for mass of 148 on P solubility was studied. For this, $9.76 \mathrm{~g}$ moist soil (equivalent to $8 \mathrm{~g}$ dry soil) were mixed with $40 \mathrm{~mL}$ distilled water or a solution of $1 \mathrm{mmol} \mathrm{L}^{-1}$ of each compound adjusted to $\mathrm{pH} 5.6$ (the $\mathrm{pH}$ of the soil) with $\mathrm{NaOH}$ or $\mathrm{HCl}$. To avoid microbial degradation, two drops of toluene were added. Each treatment was replicated three times. The samples were shaken on a reciprocal shaker at 150 cycles per minute for $5 \mathrm{~h}$. Samples were centrifuged for $15 \mathrm{~min}$ at $5000 \mathrm{rpm}$ and filtered though $0.45-\mu \mathrm{m}$ nylon membrane filters. Inorganic $\mathrm{P}$ was determined by a molybdenum blue colorimetric procedure (Murphy and Riley, 1962). 
Using a $1 \mathrm{mmol} \mathrm{L}^{-1}$ solution in a ratio of $5 \mathrm{~mL} \mathrm{~g}^{-1}$ means an addition of $5 \mu \mathrm{mol}$ compounds per $\mathrm{g}$ of soil. This quantity was estimated by using the measured exudation rate of $22 \mu \mathrm{g}$ $\mathrm{m}^{-1} \mathrm{~h}^{-1}$, and assuming this rate for 7 hours, a radius of the rhizosphere of $0.5 \mathrm{~mm}$ and a molecular weight of 148. The calculated value was increased by a factor of 5 which represents a higher exudation rate than actually measured. However, the concentration of root exudates near the root tips is higher than the average concentration. The high concentrations of compounds tested for $\mathrm{P}$ solubilising were also chosen in order to generate prominent, unequivocal effects. Fox et al. (1990) used the same concentration $\left(1 \mathrm{mmol} \mathrm{L}{ }^{-1}\right)$ and the same soil-solution ratio (1:5) for 16 organic acids to compare their ability to mobilize $\mathrm{P}$ in a Spodic Horizon Mediate.

\section{Results and discussion}

\section{Shoot dry weight and shoot $P$ concentration}

Sugar beet was grown in nutrient solution with 2 and $500 \mu \mathrm{mol} \mathrm{P} \mathrm{L}^{-1}$. The low $\mathrm{P}$ concentration resulted in a decreased yield which reached only $10-15 \%$ of the well supplied plants for each harvest (Fig. 1). Also, P concentration in shoot dry matter indicated P deficiency in low-P treatment. It was around $0.14-0.21 \%$ which is much less than the range considered as sufficient of $0.35-1.1 \%$ given by Reuter et al. (1997). The high $\mathrm{P}$ plants achieved $\mathrm{P}$ concentrations of about $1.5 \%$ in dry matter which points to a high P supply.

\section{Exudation rate}

At each harvest root exudates were collected in a trap solution over a $2 \mathrm{~h}$ period. The trap solution was lyophilized and the dry matter was determined and taken as root exudates. After measuring root length the exudation rate was calculated (Fig. 2). The P deficient plants had a 4-5 times higher exudation rate compared to the well supplied plants. These differences occurred in the same manner at all three harvests because exudation rate remained constant over the whole observed growing period. A high exudation rate at $\mathrm{P}$ deficiency - especially of carboxylates - has often been shown (Gerke et al., 2000; Neumann and Römheld, 1999, 2001; see also review of Jones, 1998). However, of more interest in this study were differences in the composition of the exudates between low-P and high-P plants. 


\section{HPLC-MS analysis of root exudates}

Full-scan HPLC-MS data were collected on root exudates as described in Materials and Methods section. The signals were normalized to compare low-P with high-P samples separately for each harvest. The normalized data were manually inspected to remove redundant signals (solvent adducts and ions containing heavy isotopes) and compared with controls to exclude background peaks. The scans of high-P and low-P exudates were compared and 56 and 9 signals were detected for negative and positive ionization mode, respectively, which were at least 5-times higher in low-P exudates compared to high-P exudates (Table 1). However, most of these signals with different intensity occurred only at one single harvest. Only 8 signals in negative mode were higher in low-P exudates in at least two harvests. Among these, two signals with $\mathrm{m} / \mathrm{z} 137$ and 147 were selected for further investigations.

\section{Phosphorus mobilization in soil}

Candidate compounds with molecular masses 138 and 148 were tested for the ability to increase P solubility in soil. Six candidate compounds were selected from KEGG database (Masoudi-Nejad et al., 2007) according to their molecular mass and functional groups, paying particular attention to carboxyls: 4-hydroxbenzoic acid, urocanic acid, salicylic acid, pantolactone, D-arabino-1,4-lactone, and citramalic acid. The effect of organic anions on the mobilization of phosphate in soil is mediated by their functional groups (Hue 1991), among which carboxyls are known to play a central role (Bolan et al., 1994). The P solubilization effect of the selected compounds is shown in Fig. 3. Only salicylic and citramalic acid (molecular weight 138 and 148, respectively) increased P concentration significantly compared to the water control. The concentration increase was by a factor of 2 and 6, respectively. The other compounds did not show any increase in P concentration in solution. This result narrowed down the list of candidate compounds to one for each HPLC-MS signal selected. 


\section{Identification of salicylic and citramalic acids in root exudates}

The mass spectrometric signals originating from root exudate components with putative molecular weights 138 and 148 were compared with the signals generated by salicylic and citramalic acids, respectively. For this comparison, samples originated from the same level of $\mathrm{P}$ supply for all three harvests were pooled to give a high-P and a low-P pool. Using reverse phase chromatography for salicylic acid and HILIC for citramalic acid (see Material and Methods), comparison of the retention times and fragmentation patterns proved that the root exudate signal with $\mathrm{m} / \mathrm{z}$ value of 137 originated from salicylic acid and the signal with $\mathrm{m} / \mathrm{z}$ value of 148 originated from citramalic acid. Salicylic and citramalic acids were present in root exudates originating from both low- and high-P treatments with concentrations in low $\mathrm{P}$ clearly higher than in high $\mathrm{P}$ (Figs. $4 \& 5$ ). The enhanced exudation of citramalic acid and salicylic acid by sugar beet roots under low P supply and the solubilization of soil $\mathrm{P}$ by these substances indicate that their function is to improve the availability of $\mathrm{P}$ to plant roots.

There are only few studies on the effect of salicylic acid on P availability in soil. Staunton and Leprice (1996) showed that salicylate significantly increased P concentration in soil solution in neutral calcic Luvisol. The competition between salicylic acid and $\mathrm{P}$ for sorption sites in two variable charge soils was documented as well by Xu et al. (2007). On the other hand, Fox et al., (1990) compared 16 organic acids and found no significant differences between salicylic acid and water in $\mathrm{P}$ solubilization. To our knowledge the exudation of citramalic acid by plant roots and its effect on $\mathrm{P}$ solubility was not yet investigated.

The different ability of salicylic and citramalic acid to mobilize $\mathrm{P}$ from soil is probably related to their functional groups and structures (aliphatic or aromatic) which affect the dissociability of carboxylic and hydroxylic groups (Fig. 6). The relative effect of different organic anions on the mobilization of phosphate from soil and metal oxides increases with the number of carboxylic groups of the anion (Bolan et al., 1994). In addition, Ae et al., (1990) demonstrated that the interrelation between the hydroxyl and carboxyl groups are important, perhaps by chelating $\mathrm{Fe}^{3+}$ in soil where most of the $\mathrm{P}$ is associated with iron (Fe-P). 
Ae et al., (1990) used a technique similar to ours to identify P mobilizing compounds in root exudates of pigeon pea. They found just one compound, i. e. piscidic acid that would be responsible for the high $\mathrm{P}$ uptake efficiency of that plant in soil containing $\mathrm{P}$ bound to Fe and Al. For sugar beet, we identified two compounds that affect $\mathrm{P}$ availability in soil. However, we studied only two among many signals enhanced under low P conditions (Table 1). Further research is therefore needed to identify compounds behind the remaining HPLC-MS signals enhanced under low-P conditions and to study their effect on the P solubility in soil.

\section{References}

Ae N, Arihara J, Okada K, Yoshihara T, Johansen C. 1990. Phosphorus uptake by pigeon pea and its role in cropping systems of the Indian subcontinent. Science 248, 477-480.

Bertin C, Yang XH, Weston LA. 2003. The role of root exudates and allelochemicals in the rhizosphere. Plant and Soil 256, 67-83.

Bhadoria PBS, Steingrobe B, Claassen N and Leibersbach H. 2002. Phosphorus efficiency of wheat and sugar beet seedlings grown in soils with mainly calcium, or iron and aluminium phosphate. Plant and Soil 264, 41-52.

Bolan NS, Naidu R, Mahimairaja S, Baskaran S. 1994. Influence of low-molecular-weight organic acids on the solubilization of phosphates. Biology and Fertility of Soils 18, $311-319$.

Dinkelaker B, Römheld V, Marschner H. 1989. Citric acid excretion and precipitation of calcium citrate in the rhizosphere of white lupine (Lupinus albus L.). Plant, Cell and Environment 12, 285-292.

Fox TR, Comerford NB, McFee WW. 1990. Phosphorus and aluminium release from a spodic horizon mediated by organic acids. Soil Science Society of America Journal 54, $1763-1767$.

Gardner WK, Barber DA, Parbery DG. 1983. The acquisition of phosphorus by Lupinus albus L. III. The probable mechanism by which phosphorus movement in the soil/root interface is enhanced. Plant and Soil 70, 107-124. 
Gerke J, Beißner L, Römer W. 2000. The quantitative effect of chemical phosphate mobilization by carboxylate anions on $\mathrm{P}$ uptake by a single root. I. The basic concept and determination of soil parameters. Journal of Plant Nutrition and Soil Science 163, 207-212.

Grimal JY, Frossard E, Morel JL. 2001. Maize root mucilage decreases adsorption of phosphate on goethite. Biology and Fertility of Soils 33, 226-230.

Hernandez G, Ramirez M, Valdes-Lopez O, Tesfaye M, Graham MA, Czechowski T, Schlereth A, Wandrey M, Erban A, Cheung F, Wu HC, Lara M, Town CD, Kopka J, Udvardi MK, Vance CP. 2007. Phosphorus stress in common bean: root transcript and metabolic responses. Plant Physiology 144, 752-767.

Hoffland E, Findenegg GR and Nelemans JA. 1989. Solubilization of rock phosphate by rape. II. Local root exudation of organic acids as a response to $\mathrm{P}$ starvation. Plant and Soil 113, 161-165.

Hue NV. 1991. Effects of organic acids/anions on P sorption and phytoavailability in soils with different mineralogies. Soil Science 152, 463-471.

Johnson JF, Allan DL, Vance CP, Weiblen G. 1996. Root carbon dioxide fixation by phosphorus deficient Lupinus albus. (Contribution to organic acid exudation by proteoid roots). Plant Physiology 112, 31-41.

Jones DL. 1998. Organic acids in the rhizosphere - a critical review. Plant and Soil 205, 2544.

Kania A, Neumann G, Cesco S, Pinton R, Römheld V. 2001. Use of plasma membrane vesicles for examination of phosphorus deficiency-induced root excretion of citrate in cluster root of white lupin (Lupinus albus L.). In: Horst WWJ, Schenk MK, Bürkert A, ed. Plant Nutrition - Food security and sustainability of agro-ecosystems through basic and applied research, Kluwer Academic Publishers, 546-547.

Kovar JL, Claassen N. 2005. Soil-root interactions and phosphorus nutrition of plants. In: Sims JT, Sharpley AN, ed. Phosphorus: Agriculture and the Environment, Madison, ASA-CSSA-SSSA, 379-414.

Liebersbach H, Steingrobe B, Claassen N. 2004. Roots regulate ion transport in the rhizosphere to counteract reduced mobility in dry soil. Plant and Soil 260, 79-88.

Lynch JM, Whipps JM. 1990. Substrate flow in the rhizosphere. Plant and Soil 129, 1-10.

Marschner H. 1995. Mineral nutrition of higher plants. Academic Press Limited, London. 
Marschner H, Römheld V. 1994. Strategies of plants for acquisition of iron. Plant and Soil 165, 261-274.

Masoudi-Nejad A, Goto S, Endo TR, Kanehisa M. 2007. KEGG bioinformatics resource for plant genomics research. Methods in Molecular Biology 406, 437-458.

Murphy J, Riley JP. 1962. A modified single solution method for the determination of phosphate in natural waters. Analytica Chimica Acta 27, 31-36.

Neumann G, Römheld V. 1999. Root excretion of carboxylic acids and protons in phosphorus-deficient plants. Plant and Soil 211, 121-130.

Neumann G, Römheld V. 2001. The release of root exudates as affected by plant's physiological status. In: Pinton R, Varanini Z, Nannipieri P, ed. The rhizosphere biochemistry and organic substances at the soil-plant interface, Marcel Decker, Inc., New York, 41-93.

Reuter JB, Edwards DG, Wilhelm NS. 1997. Temperate and tropical crops. In: Reuter DJ, Robinson JB, ed. Plant analysis: an interpretation manual, CSIRO Australia.

Shen J, Li H, Neumann G, Zhang F. 2005. Nutrient uptake, cluster root formation and exudation of protons and citrate in Lupinus albus as affected by localized supply of phosphorus in a split-root system. Plant Science 168, 837-845.

Smith CA, Want EJ, O`Maille G, Abagyan R, Suizdak G. 2006. XCMS: processing mass spectrometry data for metabolite profiling using nonlinear peak alignment, matching, and identification. Analytical Chemistry 78, 779-87.

Staunton S, Leprince F. 1996. Effect of $\mathrm{pH}$ and some organic anions on the solubility of soil phosphate: implications for P bioavailability. European Journal of Soil Science 47, $231-239$.

Whipps JM. 1990. Carbon Economy. In: Lynch JM, ed. The Rhizosphere, John Wiley and Sons, West Sussex. 59-98.

Xu RK, Xiao SC, Zhang H, Jiang J, Ji GL. 2007. Adsorption of phthalic acid and salicylic acid by two variable charge soils as influenced by sulphate and phosphate. European Journal of Soil Science 58, 335-342. 


\section{Tables and Figures}

Table 1: Number of HPLC-MS signals enhanced under low-P conditions

The criterion for the inclusion of a signal in the list was a threshold of at least 5-times enhancement of signal intensity after normalization for low $\mathrm{P}$ as compared to high-P conditions.

\begin{tabular}{cccc}
\hline Harvests & $\begin{array}{c}\text { Negative ionization } \\
(\mathrm{m} / \mathrm{z} \text { 50-400) }\end{array}$ & $\begin{array}{c}\text { Negative ionization } \\
(\mathrm{m} / \mathrm{z} 400-1000)\end{array}$ & $\begin{array}{c}\text { Positive ionization } \\
(\mathrm{m} / \mathrm{z} 50-1000)\end{array}$ \\
\hline & & Number of MS signals & \\
$1^{\text {st }}$ & 35 & 1 & 1 \\
$2^{\text {nd }}$ & 12 & 8 & 7 \\
$3^{\text {rd }}$ & 15 & 4 & 6 \\
\hline
\end{tabular}

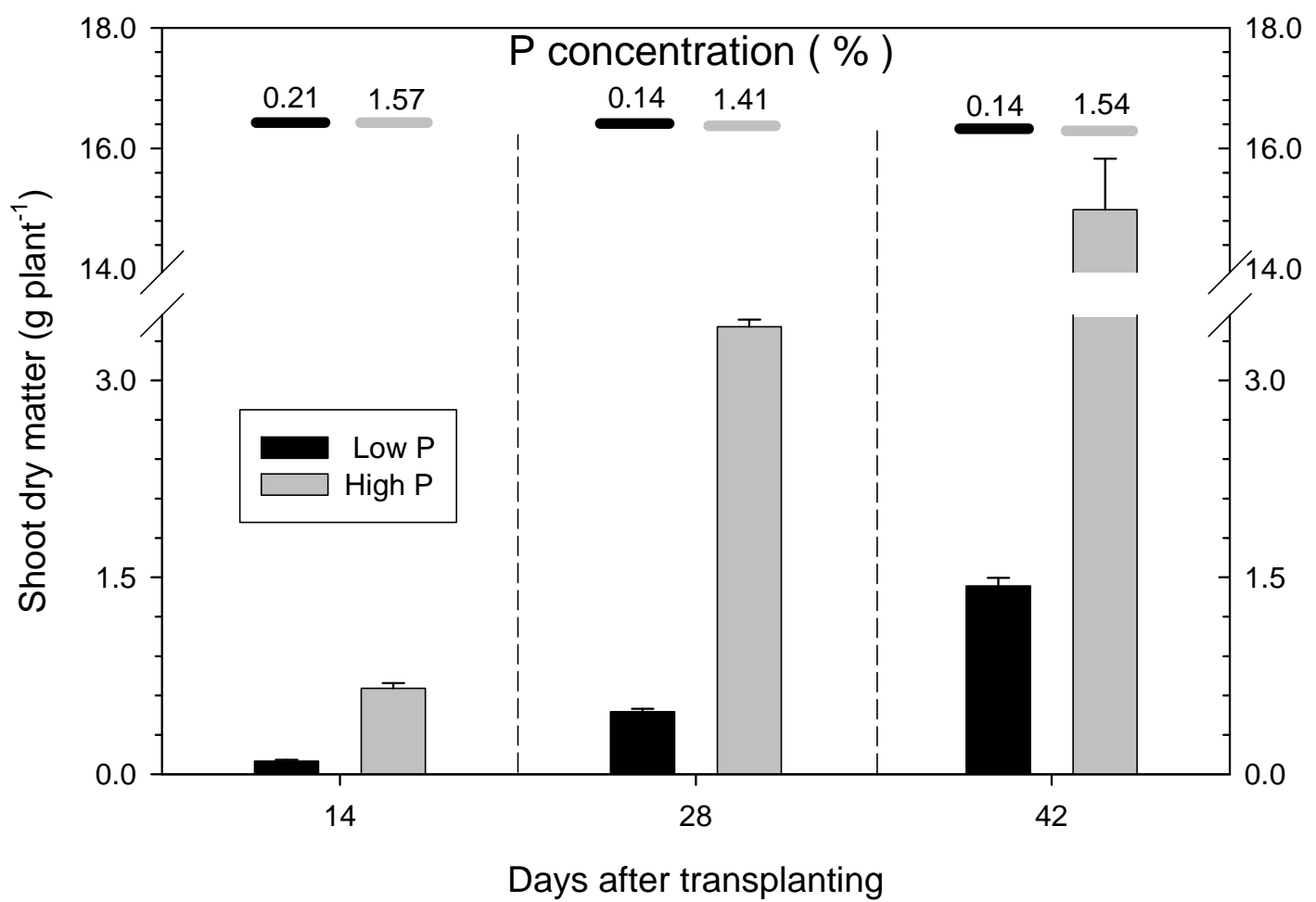

Figure 1: Shoot dry matter and $P$ concentration of sugar beet grown in nutrient solution at 2 (low $P$ ) and 500 (high $P$ ) $\mu M$ P concentration at three harvests. 


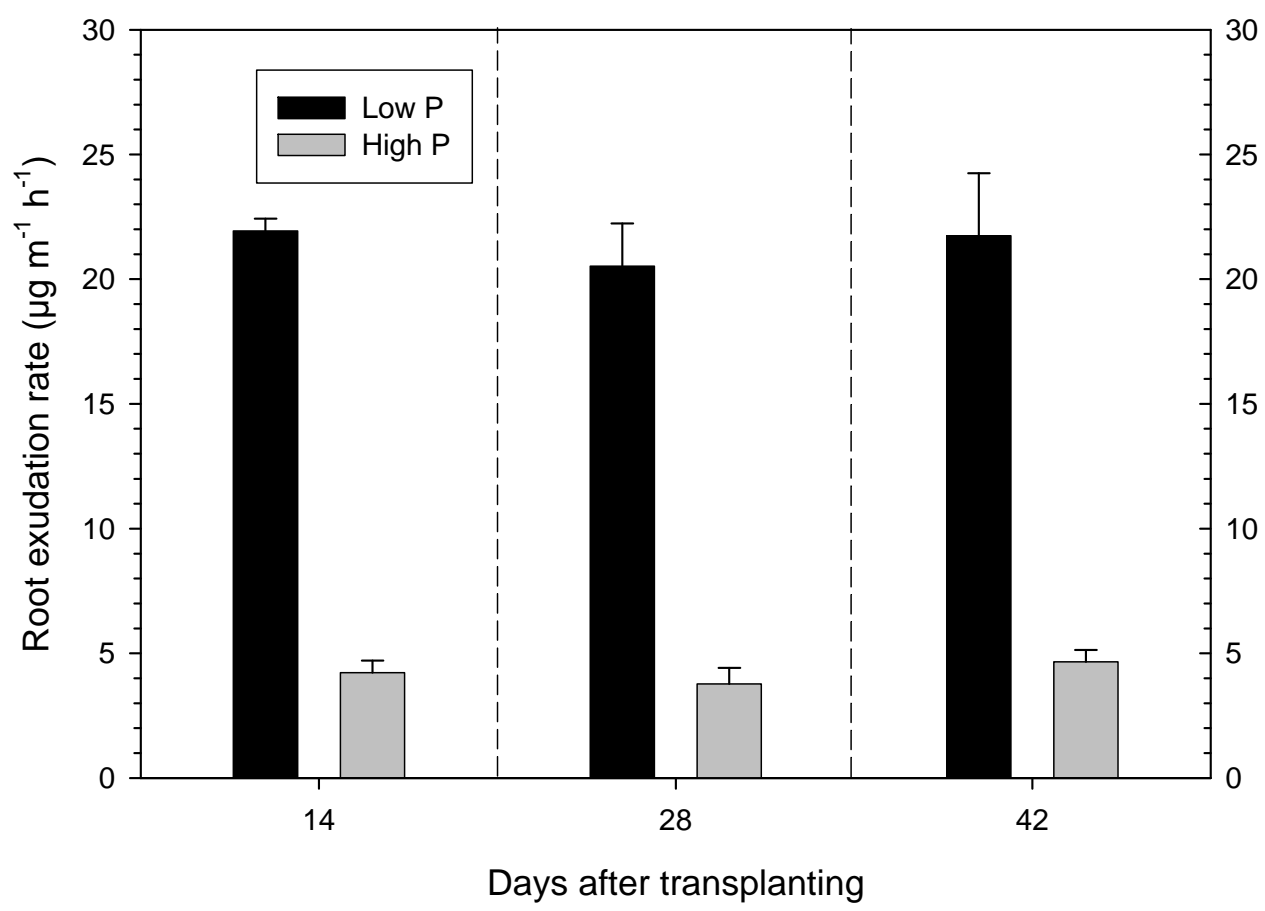

Figure 2: Rate of root exudation of sugar beet grown at low $(2 \mu \mathrm{M})$ and high $(500 \mu \mathrm{M}) \mathrm{P}$ at three consecutive harvests. 


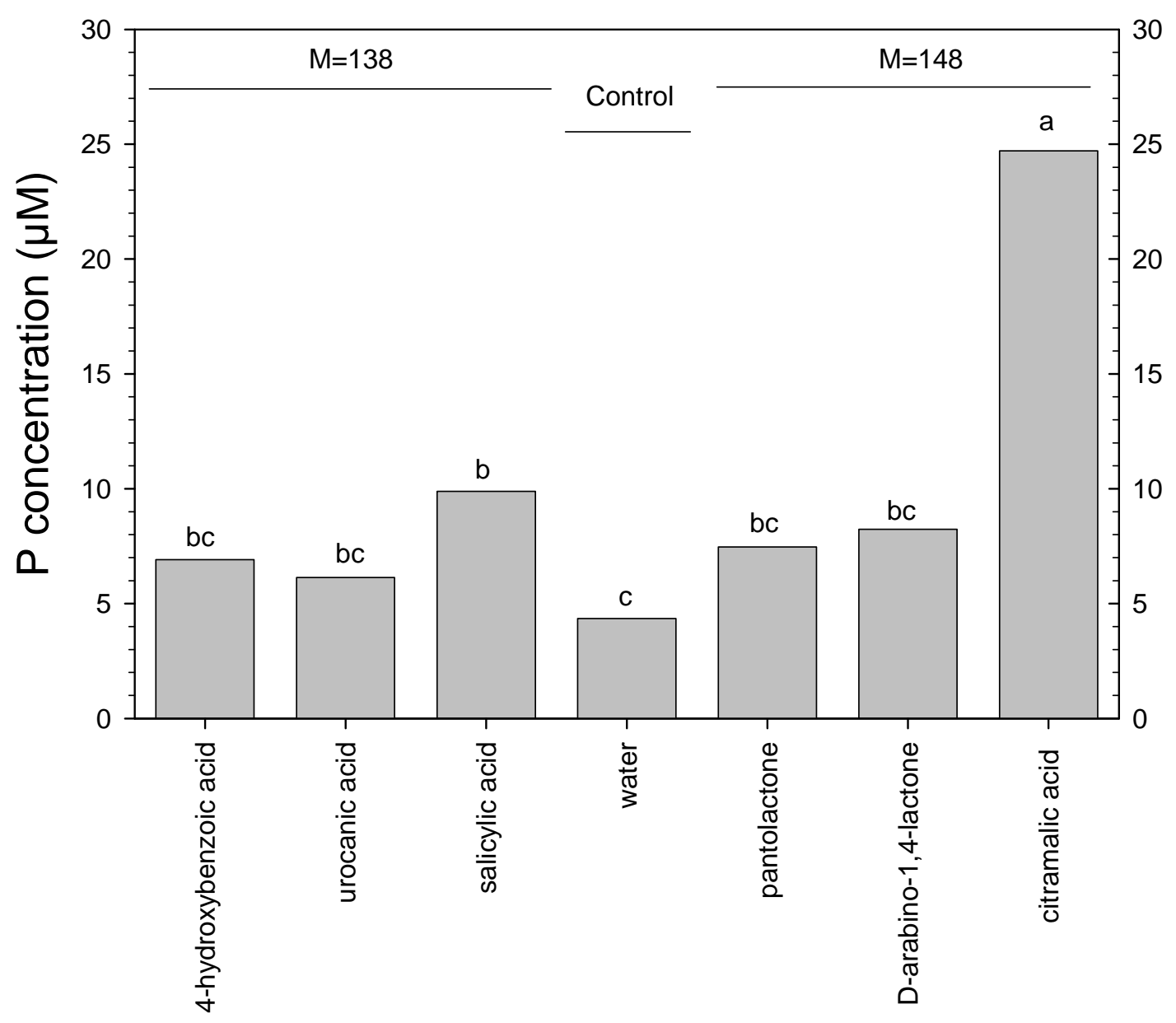

Figure 3: Effect of different substances on $P$ concentration in solution after 5 h of incubation. 


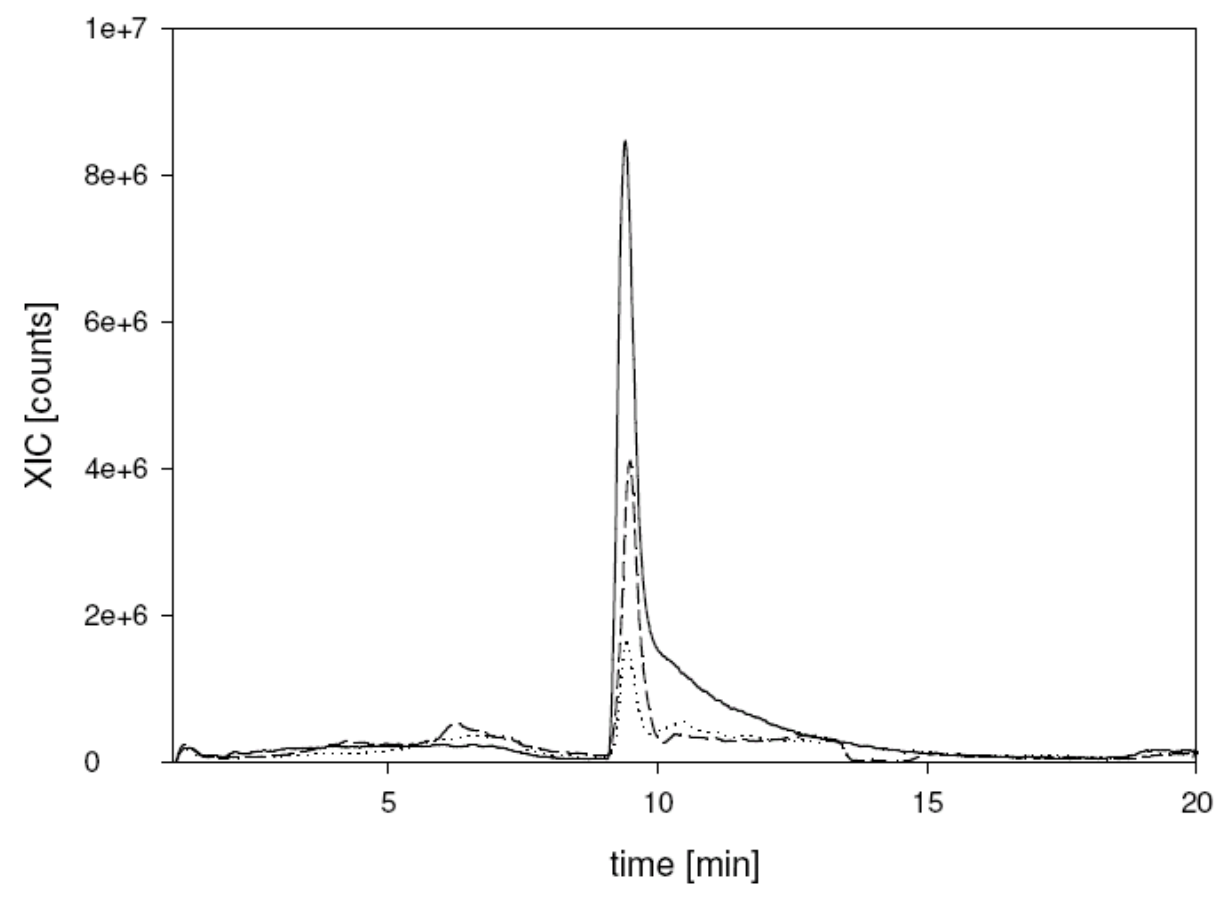

Figure 4: Extracted ion chromatogram from LC-MSMS analysis of citramalic acid standard (100 $\mathrm{ng} \mathrm{ml}^{-1}$ ) (solid line), pooled root exudates generated under low-P conditions (dashed line) and pooled root exudates generated under high-P conditions (dotted line). 


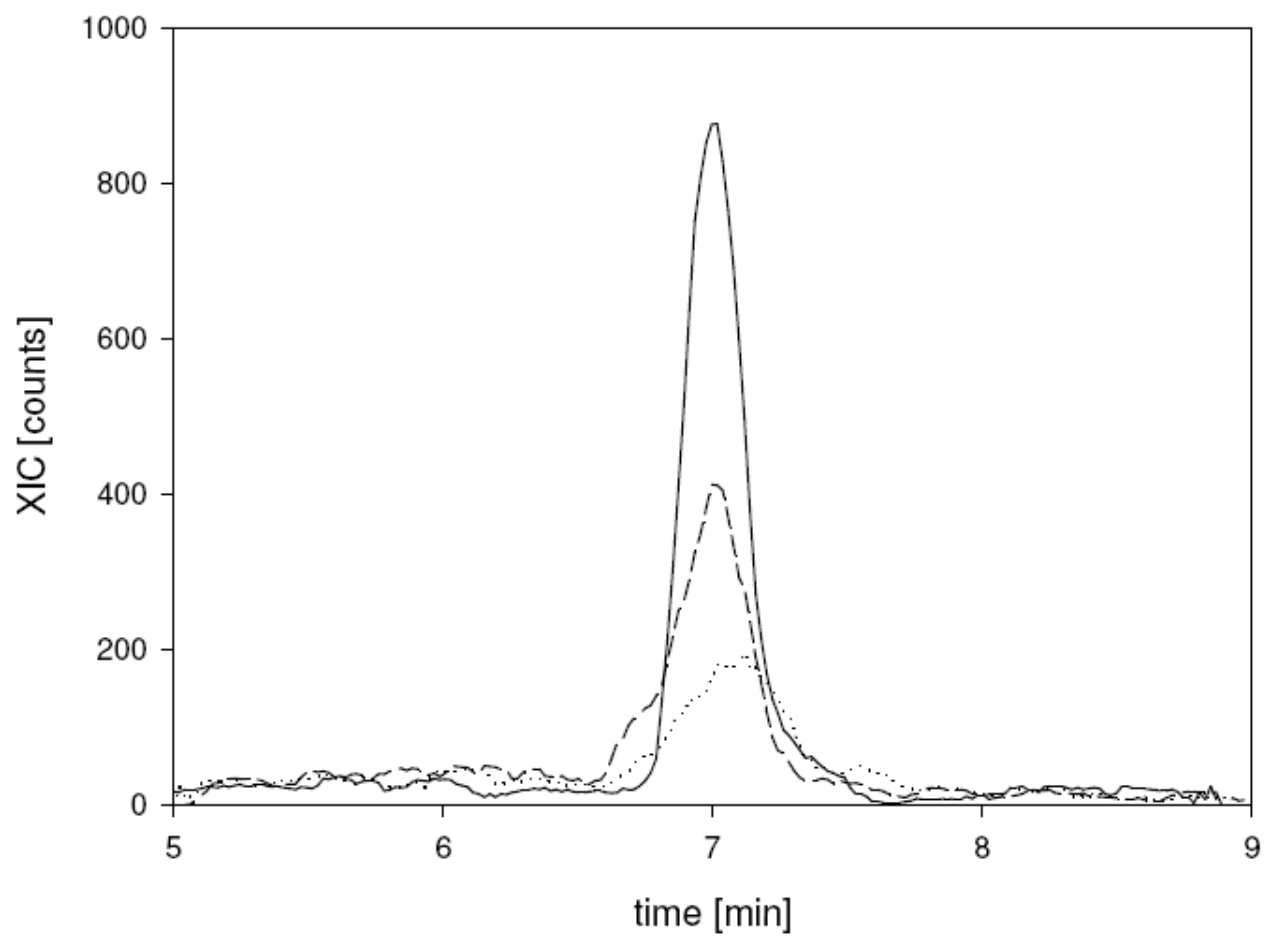

Figure 5: Extracted ion chromatogram from LC-MSMS analysis of salicylic acid standard (100 $\mathrm{ng} \mathrm{ml}^{-1}$ ) (solid line), pooled root exudates generated under low-P conditions (dashed line) and pooled root exudates (dotted line) generated under high$P$ conditions.<smiles>O=C(O)c1ccccc1O</smiles>

Salicylic acid<smiles>C[C@@H](CC(=O)O)C(=O)O</smiles>

Citramalic acid

Figure 6: Molecular structure of salicylic and citramalic acid. 


\section{Chapter 6: General Discussion}

\section{Evaluation of analytical strategies and data processing tools in application to different plant systems}

Metabolomics is still in development despite improved analytical technologies combined with new emerging bioinformatic strategies for dedicated data mining and statistics (Goodacre 2005). In this work experimental procedures and data processing routines for nontargeted differential metabolic profiling were developed and applied to different plant systems. To achieve this, a major component of the work was the assessment and adjustment of software tools for data processing.

\section{Nontargeted metabolic profiling approaches and resulting follow-up analytics}

The initial idea of unbiased metabolomics, to detect, quantify and identify all metabolites in a sample, is only possible if there are no restriction to sample preparation and analysis (Roessner et al. 2000), but up to now no single analytical methodology can achieve this aim (Kind et al. 2007). Therefore untargeted analyses are usually performed by means of metabolic profiling, to detect a broad range of chemical classes. The main advantage of untargeted metabolic approaches is that they can include the detection of unexpected and/or so far unidentified metabolites (Fiehn 2000). For these reasons, nontargeted differential metabolic profiling was used to start the investigations on the plant systems dealt within this work, i) V. longisporum/B. napus pathosystem, ii) sesame cultivars, and iii) phosphorus deficient/sufficient sugar beet, in order to obtain a survey profile of metabolites present in the sample set. Even though the studies were addressed to secondary metabolites it could not be excluded that primary metabolites were covered by the used analytical method, but they were discarded after comparative analysis at the latest, simply by the fact that they were not found to be responsible for discrimination between the sample sets. 
The quality of the samples, contributing to the quality of the corresponding LC-MS profiles, was different in each project depending on the matrix and complexity of analytes and sample variability. Sesame seed extracts were highly complex and delivered many intensive signals. The advantage of xylem sap and root exudates samples was the relative low matrix. Despite this, the largest challenges were the high biological variability of xylem sap resulting from various disease symptoms of $V$. longisporum-infected plants, and that relevant analytes occur only in trace amounts. To cope with these situations, techniques that can detect low amounts of metabolites in the noise of plant matrix using full scan mode were required. The sensitivity of a mass spectrometer in the full scan mode is mainly determined by fast scanning of a wide mass range without compromising mass resolution influencing the quality of the LC-MS profiles to a great extend. Therefore, full scan profiling was performed in the present work on a fast scanning ion trap MS, instead of using a triple quadrupole MS, which were both accessible.

The used analytical strategy for nontargeted metabolic profiling was particularly useful to identify qualitative and quantitative changes in major metabolites in sesame seed extracts according to the genotypes. It was also successfully applied to the analysis of root exudates and xylem sap, resulting in the detection of small differences even in low concentrations. Due to the discrimination of compounds expressed in trace amounts by full scan profiling follow-up studies demand for more sensitive targeted analysis.

Based on the results of the comparative analysis of metabolic profiles, different strategies were used for further analyses only on those signals that were identified to differ between samples. For instance, due to the identification of salicylic acid glucoside (SAG) in xylem sap profiles of $B$. napus infected with $V$. longisporum, the targeted quantitative analysis of phytohormones and SAG using highly selective and sensitive MRM modus on a triple quadrupole MS was initiated to check whether phytohormones play a role as signaling molecules in xylem sap in this interaction. Other mass spectrometric signals identified from xylem sap profiles to be affected by infection were subjected to structural characterization of the metabolites they come from. In the comparative experiment between root exudates released from sugar beet cultivated under low and high phosphorus concentrations, metabolic profiling set the ground for follow-up structure elucidation (performed by Dr. Ursula Hettwer) of compounds whose signals or signal intensities were found to be affected by the availability of phosphorus. Exclusively, in the investigation on 
the relationship between metabolic and genetic diversity of sesame accessions, metabolic profiling was not dedicated to select certain mass spectrometric signals for structure analysis, but rather to use them as features for sophisticated statistical correlation analysis resulting in the discrimination between genotypes (performed by Dr. Hernán Laurentin).

\section{Applicability of CODA algorithm and XCMS software for data processing}

Nontargeted metabolic profiling based on LC-MS techniques generated information-rich three-dimensional data sets (retention time $-m / z$ value - intensity). The most important step in the unbiased analysis of LC-MS based metabolic profiles is bridging the gap between recorded chromatograms (raw data) and a data format feasible to perform comparative analysis of metabolic profiles (Goodacre 2005). This step includes raw data conversion to a universal format, peak detection, retention time alignment, and peak matching. In metabolic profiling established in the present study, raw data was exported to netCDF using converter programs integrated in the software tools provided by the mass spectrometer distributor. Many software packages for unsupervised data processing are available. Two of them were tested in this work: the commercially available component detection algorithm (CODA; Windig et. al. 1996) implemented in ACDLabs /MS Manger software includes peak detection, noise reduction, and comparative data analysis. The open-source package XCMS Analyte profiling software (Smith et. al 2006) is implemented in R statistical language and performs peak detection, non linear retention time alignment, and peak matching of large data sets. Both software tools were used in dependance of the samples regarding signal-to-noise ratio $(\mathrm{S} / \mathrm{N})$ and complexity of analytes as well as matrix. The advantages of CODA method were: the fast generation of high-quality extracted ion chromatograms (EIC), the significant reduction of spectral background and the ability to perform data reduction (Fredriksson et al. 2007; Windig and Smith 2007). This can simultaneously be the major disadvantage in differential metabolic profiling. For each EIC a similarity index is calculated that declares the quality of the EIC and depending on the value of this similarity index the chromatogram is either retained in the data set or discarded. Thereby CODA is useful to find out or compare chromatograms containing peaks with a high $\mathrm{S} / \mathrm{N}$ and regarding the present work this pre-processing tool was only applicable to data generated by profiling of seed extracts. Metabolic profiles of sesame seed extracts were processed by CODA in order to find out the 10 most abundant peaks in 
metabolic profiles of each sesame accession. These peaks were combined and searched for in all accessions in the raw chromatograms to exclude discrimination of peaks by processing. Comparison of manually integrated peak areas in the raw data enabled discrimination between cultivars. However, for metabolic profiles of xylem sap it was very difficult to adjust the quality threshold without discarding relevant peaks with a poor $\mathrm{S} / \mathrm{N}$ from the data sets. This observation was also confirmed by Fredriksson et al. (2007). Therefore, the major contribution of this program to data processing is only the capability to visualize EIC which can be used to visualize EIC of masses revealed by processing using other software packages. CODA is useful to check peaks of known or previously identified masses in raw data, but is not suitable for nontargeted metabolic profiling applications requiring the detection of subtle differences.

The ratio of signal intensities (given as peak area) of relevant metabolites relative to the intensity of the TIC (total peak area) in profiles from xylem sap of B. napus as well as from root exudates from sugar beet was very low. Therefore processing of those data required software tools which can detect also trace signals. During this $\mathrm{PhD}$ work, a new open-source method for peak detection, non linear retention time alignment of LC-MS data, and peak matching across samples was provided by (Smith et al. 2006). This program called XCMS reduces three-dimensional raw data to two dimensions by combining mass information and retention time in one feature identifying a peak. This software is very suitable for the comparison of large profile sets and uses a t-test and $p$ value to rank important peaks. XCMS processing is very simple to perform on the raw data. It is only necessary to classify the samples into groups which will be compared. There are several parameters that can be adjusted by the user, but most parameters were appropriate for raw data processing already in the default settings. Alterations of the parameter settings resulted only in minimal changes on the total number of detected peaks and reproducibility of peak area. This was recently recommended by other users, too (Dunn et al. 2008). The detection of peaks with low intensities is reliable, but occasionally XCMS integrates noise which hampers further comparative analysis. Another drawback was that in different combinations of data sets, peak picking and the peak areas were not exactly reproducible. For these reasons, results of XCMS processing have to be re-checked manually in the raw data, which was also recommended by Kind et al. (2007). 
In this work XCMS was firstly applied in plant metabolomics for processing metabolic profiles of xylem sap and sugar beet root exudates.

One single LC-MS profiling data file has about 30MB in size. Processing of large data sets by XCMS as well as CODA requires fast hardware for time-consuming deconvolution and alignment processes. Therefore Kind et al. (2007) used a computer equipped with $(2.8 \mathrm{GHz}$ and 4 GB memory).

\section{Normalization}

Normalization protocol described in this work (see chapter 2) was applied to xylem sap and sugar beet root exudate samples and is based on the data itself. It therefore requires many detected signals, which are only available from full scan mode, but not from SIM or MRM techniques. The application of our protocol relies on the majority of peaks which are unchanged between comparing samples, excluding signals affected by pathogen infection or regulated by P-deficiency from the calculation of the normalization factor. It is therefore applicable for samples originated from plant-pathogen interaction or a treatment experiment, such as different phosphorus supply, because only few signals change after treatment. For the normalization of metabolic profiles from accessions of sesame, this normalization scheme could not be used, because of too much variation on metabolite levels and no adequate data format was available from CODA for normalization. However, reliable and reproducible sample preparation was demonstrated in Chapter 4, sesame data did not underlie high variations described for xylem sap.

\section{Characterization of metabolites structures}

Data depending scanning (DDS) feature is a valuable tool for structural characterization. It scans alternate between a survey in fullscan mode and stepwise $\mathrm{MS}^{\mathrm{n}}$ fragmentation. The combination metabolic profiling in full scan and data dependent scanning in parallel could be useful for on-line structural characterization of unknown or known metabolites in metabolomic approaches. But, the drawback is that the data format is not suitable for data processing with XCMS or CODA algorithm, since data from $\mathrm{MS}^{\mathrm{n}}$ fragmentation can not be separated from full scan profiling data. Furthermore, an important point is that the DDS parameters have to be optimized carefully to the needs of the sample e.g., xylem sap 
analysis demands special requirement. This feature on the ion trap mass spectrometer was applied for follow-up structure characterization of signals identified by comparative analysis of metabolic profiles from xylem sap of B. napus/V. longisporum interaction and sugar beet root exudates.

\section{Putative structures of signals in xylem sap of B. napus after $V$. longisporum infection}

The main objective in this study was the application of the differential metabolic profiling procedure established in this work to elucidate metabolic changes in the chemical composition of xylem sap during B. napus/ $V$. longisporum interaction.

The structures of metabolic signals affected by infection as revealed by comparative analysis of metabolic profiles of xylem sap were characterized using mass spectrometric fragmentation (see Chapter 2 and 3).

As the main results from studying this interaction, the characterization of signals revealed the presence of salicylic acid and its glucoside, in xylem sap of B. napus and its elevation after infection with $V$. longisporum. Both are known signaling molecules involved in general plant defense (Glazebrook 2005). Apart from this, six infection-specific signals were found and are assumed to play a role in more specific plant defense. Since metabolite intensities were correlated among infection-specific signals it is assumed that they derive from the same pathway. Their common structure motifs are indole-3-carboxylic acid and tryphtophan. The putative structures of fragment ions and neutral losses as revealed by mass spectrometric fragmentation (shown in Tab. 1) occur in tryptophan pathway as shown in Fig. 1. Tryptophan is known as precursor and building block of phytoalexins and glucosinolates in Brassica spp. and other crucifers, but infection-specific metabolites detected in xylem sap appear to be compounds not identified so far. Indole-3-carboxylic acid esters have been reported to be induced in species-specific defense of A. thaliana (Hagemeier et al. 2001). The infection-specific signals identified in xylem sap of B. napus infected with $V$. longisporum play a crucial role in this interaction. A possible function as plant defense chemicals can be assumed but remains to be elucidated by further studies. 
Tab.1: Characterization of infection-specific signals by MS fragmentation.

Structure suggestions for detected fragments and neutral losses occurring in $\mathrm{MS}^{\mathrm{n}}$ fragmentation of infection-specific signals are given.

\begin{tabular}{cccl}
$\begin{array}{c}{[\mathrm{M}-\mathrm{H}]^{-}} \\
(\mathrm{m} / \mathrm{z})\end{array}$ & $\begin{array}{c}{[\mathrm{M}+\mathrm{H}]^{+}} \\
(\mathrm{m} / \mathrm{z})\end{array}$ & {$[\mathrm{M}]$} & Putative structures \\
& & 44 & $\mathrm{CO}_{2}$ \\
& 118 & 117 & Indole \\
& 146 & 145 & Indole-3-carboxaldehyde \\
145 & & 146 & Glutamine/ lysine/ 2-oxoglutarate \\
148 & & 149 & 5,6-Dihydroxyindole \\
& 162 & 161 & Indole-3-carboxylic acid / indol-3-ethanol \\
& & 162 & Glucoside, hexoside \\
& & 180 & Glucose or other sugar \\
174 & & 175 & Indole-3-acetic acid/3-indoleglycolaldehyd/ \\
& & & 5-Hydroxyindoleacetaldehyde \\
192 & & 193 & 5,6-Dihydroxyindole-2-carboxylate \\
& & 202 & alpha,beta-didehydrotryptophan \\
202 & & 203 & Indole-3-pyruvate \\
& & 204 & Trytophan \\
& & 248 & 5-Hydroxyindoleacetylglycine \\
& 324 & 323 & ß-D-Glucopyranosyl indole-3-carboxylic acid \\
\hline
\end{tabular}

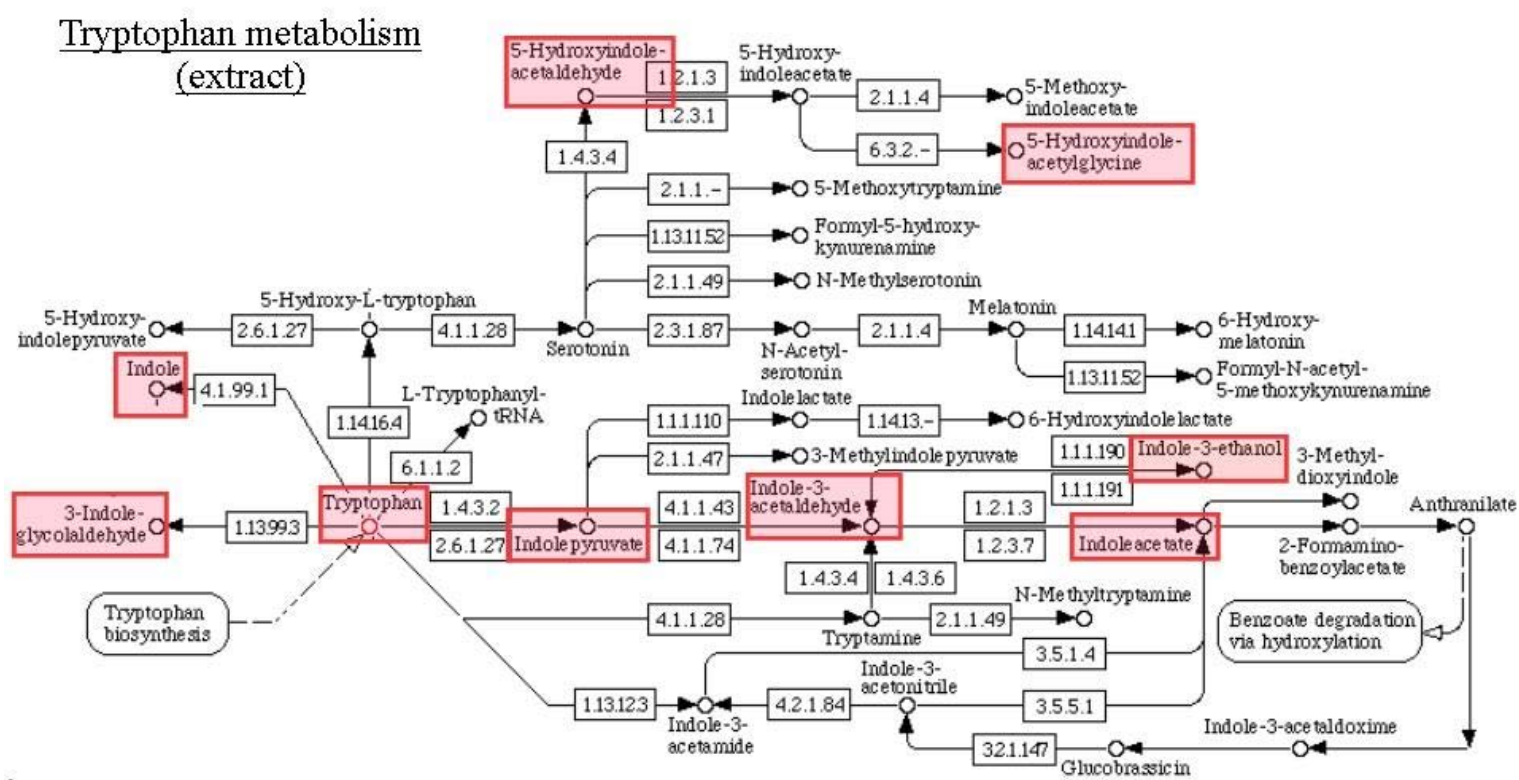

Figure 1: Metabolic pathway map adapted to KEGG database of metabolites that are putative building blocks of infection-specific signals found in xylem sap from Brassica napus after $V$. longisporum infection. 


\section{Conclusion}

In the present work, nontargeted metabolic profiling based on LC-MS was developed for comparative experiments in plant systems. In the new established data processing routine open-source method XCMS for peak detection and retention time alignment from raw data was included, which has been proved to be a powerful tool, applicable to plant metabolomics enabling the detection of low abundant signals in a complex plant matrix. In contrast, CODA algorithm is not suitable for unbiased data analysis of nontargeted profiles, but provides the visualization of results obtained from XCMS. An algorithm for normalization of signal intensities, previously developed for transcriptomics, was successfully implemented in data processing.

The applicability of the nontargeted metabolic profiling routine was demonstrated on plant-pathogen interaction: small molecules changes in xylem sap during the interaction Brassica napus/Verticillium longisporum were elucidated; firstly salicylic acid and its glucoside, and secondly six putative yet unknown indolic polar metabolites, all might be involved in the plant defense response. Their structure elucidation requires purification of xylem sap.

Metabolic profiling can reveal mechanisms in plant response to nutrient deficiency. From nontargeted metabolic profiles of root exudates from phosphorus deficient sugar beets selected signals were dedicated to follow-up structure analysis which revealed salicylic acid and citramalic acid.

In combination with genome diversity data on sesame metabolic profiling of sesame seeds was demonstrated to provide valuable information on sesame diversity which can be used in breeding. 


\section{Summary}

Metabolic profiling helps to understand biological processes responsible for differences in the content of metabolites among samples, such as healthy/diseased organisms, treated/control cultures, mutant/wildtype genotypes, or different cultivars. The goal of the $\mathrm{PhD}$ project was to develop experimental procedures and data processing routines suitable for the application of nontargeted metabolic profiling in plant systems. The analysis generated information-rich three-dimensional data sets (retention time- $\mathrm{m} / \mathrm{z}$ ratio intensity). An important component of the work was the assessment and adjustment of software tools for data processing (noise reduction, chromatogram alignment, peak matching, normalization of intensities and comparative analysis).

One major objective of the work was the investigation of the pathosystem Brassica napus/ Verticillium longisporum. Chemical interactions between plant and fungus are confined to vascular tissue, which required extraction and analysis of xylem sap. Development of a protocol for normalization of signal intensities for this kind of samples was an important part of data processing.

Filtered xylem sap was separated on polar modified reverse phase column and detected in positive and negative full scan modes on a fast scanning ion trap MS. Data processing included noise reduction, peak alignment methods XCMS or CODA, normalization of signal intensities and comparative analysis.

By comparative analysis of the normalized metabolic profiles, 19 signals were identified whose intensities change after pathogen infection. Selected signals were monitored in timecourse experiments, at different infection levels and in different plant organs. Secondary metabolites involved in the interaction were characterized by $\mathrm{MS}^{\mathrm{n}}$-experiments.

Signals can be divided into three classes: enhanced, suppressed and infection-specific. Six signals (infection-specific) only occurred in xylem of plants infected with $V$. longisporum but neither in healthy plants nor in plants infected with Verticillium dahliae.

Characterization of infection-specific signals by $\mathrm{MS}^{\mathrm{n}}$ fragmentation revealed putative building blocks composed of indole carboxylic acid, tryptophan and indole pyruvate as common motif of their structure. The levels of infection-specific signals within different xylem sap pools were correlated, suggesting that they derive from the same pathway. Some of the infection-specific signals correlate with fungal biomass in the plant tissue in single 
plant analysis and during the time-course. The structures of metabolites specific occurring in xylem sap of $V$. longisporum-infected B. napus plant appear to be novel.

One of xylem metabolites enhanced by infection was identified as salicylic acid glucoside. Salicylic acid and its glucoside present in xylem sap shows elevated levels after infection with $V$. longisporum, too. The concentration of SAG correlated both with plant height reduction and fungal DNA in plant tissue quantified by species-specific real-time PCR.

Apart from the investigation of Brassica/Verticillium interaction, the metabolic profiling protocol was extended for the comparison of more than two kinds of samples, as required in the investigation of the chemical diversity in sesame (Sesamum indicum L; samples generated by Dr. Hernán Laurentin). Here, metabolic profiles were used to address the effect of selection and genetic drift on the evolution of diversity, without the need to characterize every analytical signal. Furthermore, the protocol was applied to the analysis of phosphorus- mobilizing root exudates of sugar beet (samples generated by Dr. Reza Khorassani). Differential metabolic profiling of root exudates obtained under low phosphorus and high phosphorus conditions revealed that several signals were enhanced under low-P conditions as compared to high-P. In this project metabolic profiling set the ground for follow-up structure elucidation of signals identified as different and therefore assumed to be involved in phosphorus acquisition. 


\section{References (cited in general introduction and general discussion)}

Aharoni A, Ric de Vos CH, Verhoeven HA, Maliepaard CA, Kruppa G, Bino R, Goodenowe DB (2002) Nontargeted metabolome analysis by use of Fourier Transform Ion Cyclotron Mass Spectrometry. OMICS 6(3):217-234

Allwood JW, Ellis DI, Heald JK, Goodacre R, Mur LAJ (2006) Metabolomic approaches reveal that phosphatidic and phosphatidyl glycerol phospholipids are major discriminatory non-polar metabolites in responses by Brachypodium distachyon to challenge by Magnaporthe grisea. Plant J 46(3):351-368

Allwood JW, Ellis DI, Goodacre R (2008) Metabolomic technologies and their application to the study of plants and plant-host interactions. Physiol Plant 132(2):117-135

Beckmann CH (1987) The Nature of Wilt Diseases of Plants. St. Paul, MN, USA: APS Press

Bednarek P, Schneider B, Svatos A, Oldham NJ, Hahlbrock K (2005) Structural complexity, differential response to infection, and tissue specificity of indolic and phenylpropanoid secondary metabolism in Arabidopsis roots. Plant Physiol 138(2):10581070

Bell AA (1969) Phytoalexin production and Verticilllium wilt resistance in cotton. Phytopathology 59:1119-1127

Benhamou N (1995) Ultrastructural and cytochemical aspects of the response of eggplant parenchyma cells in direct contact with Verticillium infected xylem vessels. Physiol Mol Plant Pathol 46:321-338 
Bino RJ, Ric de Vos CH, Lieberman M, Hall RD, Bovy A, Jonker HH, Tikunov Y, Lommen A, Moco S, Levin I (2005) The light-hyperresponsive high pigment-2dg mutation of tomato: alterations in the fruit metabolome. New Phytol 166(2):427-438

Birkemeyer C, Luedemann A, Wagner C, Erban A, Kopka J (2005) Metabolome analysis: the potential of in vivo labeling with stable isotopes for metabolite profiling. Trends Biotechnol 23(1):28-33

Bishop CD and Cooper RM (1983) An ultrastructural study of root invasion in three vascular wilt diseases I. Colonization of susceptible cultivars. Physiol Plant Pathol 23:323343

Buchner V, Nachmias A, Burstein Y (1982) Isolation and partial characterization of a phytotoxic glycopeptide from a proteinlipopolysaccharide complex produced by a potato isolate of Verticillium dahliae. FEBS Lett 138:261-264

Buchner V, Burnstein Y, Nachmias A (1989) Comparison of Verticillium dahliae produced phytotoxic peptides purified from culture fluids and infected potato stems. Physiol Mol Plant Pathol 35:253-269

Carder JH, Hignett RC, Swinburne TR (1987) Relationship between the virulence of hop isolates of Verticillium albo-atrum and their in vitro secretion of cell-wall degrading enzymes. Physiol Mol Plant Pathol 31:441-452

Chiwocha SD, Abrams SR, Ambrose SJ, Cutler AJ, Loewen M, Ross AR, Kermode AR (2003) A method for profiling classes of plant hormones and their metabolites using liquid chromatography-electrospray ionization tandem mass spectrometry: an analysis of hormone regulation of thermodormancy of lettuce (Lactuca sativa L.) seeds. Plant $\mathrm{J}$ $35(3): 405-417$

Cooper RM, Resende MLV, Flood J, Rowan MG, Beale MH, Potter U (1996) Detection and cellular localization of elemental sulphur in disease resistant genotypes of Theobroma cacao. Nature 379:159-162 
Costacurta A and Vanderleyden J (1995) Synthesis of phytohormones by plant-associated bacteria. Crit Rev Microbiol 21:1-18

Cramer RA and Lawrence CB (2004) Identification of Alternaria brassicicola genes expressed in planta during pathogenesis of Arabidopsis thaliana. Fungal Genet Biol 41(2):115-128

Daayf F, Nicole M, Boher B, Pando A, Geiger JP (1997) Early vascular defense reactions of cotton roots infected with defoliating mutant strain of Verticillium dahliae. Eur J Plant Pathol 103:125-136

Davis DA, Low PS, Heinstein P (1998) Purification of a glycoprotein elicitor of phytoalexin formation from Verticillium dahliae. Physiol Mol Plant Pathol 52:259-273

Del Sorbo G, Schoonbeek H, De Waard MA (2000) Fungal transporters involved in efflux of natural toxic compounds and fungicides. Fung Gen Biol 30:1-15

Demain AL and Fang A (2000) The natural functions of secondary metabolites. Adv Biochem Eng Biotechnol 69:1-39

Desjardins AE, Hohn TM, McCormick SP (1993) Trichothecene biosynthesis in Fusarium species: chemistry, genetics, and significance. Microbiol Rev 57(3):595-604

Dixelius C, Happstadius I, Berg G (2005) Verticillium wilt on Brassica oil crops - a swedish perspective. Journal of the Swedish Seed Association 115:36-48

Dixon RA (2001) Natural products and plant disease resistance. Nature 411:843-847

Dunn WB, Broadhurst D, Brown M, Baker PN, Redman CW, Kenny LC, Kell DB (2008) Metabolic profiling of serum using Ultra Performance Liquid Chromatography and the LTQ-Orbitrap mass spectrometry system. J Chromatogr B Analyt Technol Biomed Life Sci doi:10.1016/j.jchromb.2008.03.021 
Duran AL, Yang J, Wang L, Sumner LW (2003) Metabolomics spectral formatting, alignment and conversion tools (MSFACTs). Bioinformatics 19(17):2283-2293

Durgbanshi A, Arbona V, Pozo O, Miersch O, Sancho JV, Gómez-Cadenas A (2005) Simultaneous determination of multiple phytohormones in plant extracts by liquid chromatography-electrospray tandem mass spectrometry. J Agric Food Chem 53(22):84378442

Durrands PK and Cooper RM (1988) The role of pectinases in vascular wilt disease as determined by defined mutants of Verticillium albo-atrum. Physiol Mol Plant Pathol $32: 363-371$

Eynck C, Koopmann B, Grunewaldt-Stoecker G, Karlovsky P, von Tiedemann A (2007) Differential interactions of Verticillium longisporum and $V$. dahliae with Brassica napus detected with molecular and histological techniques. Eur J Plant Pathol 118:259-274

Fahey JW, Zalcmann AT, Talalay P (2001) The chemical diversity and distribution of glucosinolates and isothiocyanates among plants. Phytochemistry 56:5-51

Fan TW, Lane AN, Shenker M, Bartley JP, Crowley D, Higashi RM (2001) Comprehensive chemical profiling of gramineous plant root exudates using high-resolution NMR and MS. Phytochemistry 57(2):209-221

Fiehn O, Kopka J, Dörmann P, Altmann T, Trethewey RN, Willmitzer L (2000) Metabolite profiling for plant functional genomics. Nat Biotechnol 18(11):1157-1161

Fiehn O (2002) Metabolomics - the link between genotypes and phenotypes. Plant Mol Biol 48(1-2):155-171

Fradin EF and Thomma BPHJ (2006) Physiology and molecular aspects of Verticillium wilt diseases caused by V.dahliae and V. albo-atrum. Molecular Plant Pathology 7:71-86 
Fredriksson M, Petersson P, Jörntén-Karlsson M, Axelsson BO, Bylund D (2007) An objective comparison of pre-processing methods for enhancement of liquid chromatography-mass spectrometry data. J Chromatogr A 1172(2):135-50

Fry W and Myers D (1981) Hydrogen cyanide metabolism by fungal pathogens of cyanogenic plants. In: Cyanide in biology (Vennesland B, Knowles C, Conn E, Westley J and Wissing F, eds), Academic Press, UK, pp. 321-334

Glazebrook J (2005) Contrasting mechanisms of defense against biotrophic and necrotrophic pathogens. Annu Rev Phytopathol 43:205-227

Gold J and Robb J (1995) The role of the coating response in Craigella tomatoes infected with Verticillium dahliae, races 1 and 2. Physiol Mol Plant Pathol 47:141-157

Gold J, Lee B, Robb J (1996) Colonization of tomatoes by Verticillium dahliae: determinative phase II. Canadian Journal of Botany 74:1279-1288

Goodacre R (2005) Making sense of the metabolome using evolutionary computation: seeing the wood with the trees. J Exp Bot 56(410):245-254

Goto S, Okuno Y, Hattori M, Nishioka T, Kanehisa M (2002) LIGAND: database of chemical compounds and reactions in biological pathways. Nucleic Acids Res 30(1):402404

Grayer RJ and Kokubun T (2001) Plant-fungal interactions: the search for phytoalexins and other antifungal compounds from higher plants. Phytochemistry 56(3):253-263

Guerinot ML (1994) Microbial iron transport. Annu Rev Microbiol 48:743-772

Hagemeier J, Schneider B, Oldham NJ, Hahlbrock K (2001) Accumulation of soluble and wall-bound indolic metabolites in Arabidopsis thaliana leaves infected with virulent or avirulent Pseudomonas syringae pathovar tomato strains. Proc Natl Acad Sci U S A 98(2):753-758 
Hahlbrock K and Scheel D (1989) Physiology and molecular biology of phenylpropanoid metabolism. Annu Rev Plant Physiol Plant Mol Biol 40:347-369

Hahlbrock K, Bednarek P, Ciokowski I, Hamberger B, Heise A, Liedgens H, Logemann E, Nurnberger T, Schmelzer E, Somssich IE, Tan J (2003) Non-self recognition, transcriptional reprogramming, and secondary metabolite accumulation during plant/pathogen interaction. Proc Natl Acad Sci U S A 100:14569-14576

Hall RD, Vos CHR, Verhoeven HA, Bino RJ (2005) Metabolomics for the assessment of functional diversity and quality traits in plants. In: Vaidyanathan S, Harrigan GG, Goodacre R, eds. Metabolome analyses: strategies for systems biology. New York, NY, USA. Springer

Hall RD (2006) Plant metabolomics: from holistic hope, to hype, to hot topic. New Phytol 169(3):453-468

Hammerschmidt R (1999) Phytoalexins: what have we learned after 60 years? Annu Rev Phytopathol 37:285-306

Happstadius I, Ljunberg A, Kristiansson B, Dixelius C (2003) Identification of Brassica oleracea germplasm with improved resistance to Verticillium wilt. Plant Breeding 122:3034

Hause B, Mrosk C, Isayenkov S, Strack D (2007) Jasmonates in arbuscular mycorrhizal interactions. Phytochemistry 68(1):101-110

Hernández G, Ramírez M, Valdés-López O, Tesfaye M, Graham MA, Czechowski T, Schlereth A, Wandrey M, Erban A, Cheung F, Wu HC, Lara M, Town CD, Kopka J, Udvardi MK, Vance CP (2007) Phosphorus stress in common bean: root transcript and metabolic responses. Plant Physiol 144(2):752-767

Hof H (2008) Mycotoxins: pathogenicity factors or virulence factors? Mycoses. 51(2):9394 
Huang CY, Roessner U, Eickmeier I, Genc Y, Callahan DL, Shirley N, Langridge P, Bacic A (2008) Metabolite profiling reveals distinct changes in carbon and nitrogen metabolism in phosphate-deficient barley plants (Hordeum vulgare L.). Plant Cell Physiol 49(5):691703

Huhman DV and Sumner LW (2002) Metabolic profiling of saponins in Medicago sativa and Medicago truncatula using HPLC coupled to an electrospray ion-trap mass spectrometer. Phytochemistry 59(3):347-360

Jahangir M, Kim HK, Choi YH, Verpoorte R (2008) Metabolomic response of Brassica rapa submitted to pre-harvest bacterial contamination. Food Chemistry 107(1):362-368

Johansson A, Staal J, Dixelius C (2006) Early responses in the Arabidopsis-Verticillium longisporum pathosystem are dependent on NDR1, JA- and ET-associated signals via cytosolic NPR1 and RFO1. Mol Plant Microbe Interact 19:958-969

Johnson HE, Broadhurst D, Goodacre R, Smith AR (2003) Metabolic fingerprinting of salt stressed tomatoes. Phytochemistry 62:919-928

Kanehisa M, Goto S, Kawashima S, Nakaya A (2002) The KEGG databases at GenomeNet. Nucleic Acids Res 30(1):42-46

Kaplan F, Kopka J, Haskell DW, Zhao W, Schiller KC, Gatzke N, Sung DY, Guy CL (2004) Exploring the temperature stress metabolome of Arabidopsis. Plant Physiol $136: 4159-4168$

Karapapa VK, Bainbridge BW, Heale JB (1997) Morphological and molecular characterisation of Verticillium longisporum comb. nov., pathogenic to oilseed rape. Mycological Research 101:1281-1294

Katajamaa M and Oresic M (2005) Processing methods for differential analysis of LC/MS profile data. BMC Bioinformatics 6:179 
Keen NK, Long M, Erwin DC (1972) Possible involvement of a pathogen-produced protein lipopolysaccharide complex in Verticillium wilt of cotton. Physiol Plant Pathol 2:317-331

Kind T, Tolstikov V, Fiehn O, Weiss RH (2007) A comprehensive urinary metabolomic approach for identifying kidney cancer. Anal Biochem. 2007 Apr 15;363(2):185-95.

Kind $\mathrm{T}$ (2008) Peak Alignment of LC, GC, MS, NMR data URL: http://fiehnlab.ucdavis.edu/staff/kind/Metabolomics/Peak_Alignment (date 12/05/2008)

Kopka J, Fernie A, Weckwerth W, Gibon Y, Stitt M (2004) Metabolite profiling in plant biology: platforms and destinations. Genome Biol 5(6):109

Kossel A (1891) Ueber die Chemische Zusammensetzung der Zelle. Arch Physiol 181-186

Krasilnikov NA, Khodzhibaeva SM, Mirchink TG (1969) Properties of toxins of $V$. dahliae, the causative agent of cotton wilt disease. J. Gen Appl Microbiol 15:1-9

Kutchan TM (2001) Ecological arsenal and developmental dispatcher. The paradigm of secondary metabolism. Plant Physiol 125(1):58-60

Lee SW, Nazar RN, Powell DA, Robb J (1992) Reduced PAL gene suppression in Verticillium-infected resistant tomatoes. Plant Mol Biol 18(2):345-352

Mansoori B, Milton JM, Smith CJ (1995) Isolation and partialpurification of a phytotoxin related to pathogenic Verticillium species. J Phytopathol 143:33-36

Mansoori B and Smith CJ (2005) Elicitation of ethylene by Verticillium albo-atrum phytotoxins in potato. J Phytopathol 153:143-149

Memelink J (2005) Tailoring the plant metabolome without a loose stitch. Trends in Plant Science 7:305-307 
Meyer R, Slater V, Dubery LA (1994) A phytotoxic proteinlipopolysaccharide complex produced by Verticillium dahliae. Phytochemistry 35:1449-1453

Mol L and Scholte K (1995) Formation of microsclerotia of Verticillium dahliae Kleb. on various plant parts of two potato cultivars. Potato Research 38:143-150

Mueller A, Duechting P, Weiler EW (2002) A multiplex GC-MS/MS technique for the sensitive and quantitative single-run analysis of acidic phytohormones and related compounds, and its application to Arabidopsis thaliana. Planta 216(1):44-56

Nachmias A, Buchner V, Burstein Y (1985) Biological and immunochemical characterization of a low molecular weight phytotoxin isolated from a proteinlipopolysaccharide complex produced by a potato isolate of Verticillium dahliae Kleb. Physiol Plant Pathol 26:43-55

Nachmias A, Buchner V, Tsror L, Burstein Y, Keen N (1987) Differential phytotoxicity of peptides from culture fluids of Verticillium dahliae races 1 and 2 and their relationship to pathogenecity of the fungi on tomato. Phytopathology 77:506-510

Ng KM, Zhitao L, Wei L, Ho-Wai T, Zhongzhen Z, Chi-Ming C, Yung-Chi C (2007) In Vivo Analysis and Spatial Profiling of Phytochemicals in Herbal Tissue by MatrixAssisted Laser Desorption/Ionization Mass Spectrometry. Anal Chem79:2745-2755

Niessen WM (1999) State-of-the-art in liquid chromatography-mass spectrometry. J Chromatogr A 856(1-2):179-197

Nikiforova VJ, Kopka J, Tolstikov V, Fiehn O, Hopkins L, Hawkesford MJ, Hesse H, Hoefgen R (2005) Systems rebalancing of metabolism in response to sulfur deprivation, as revealed by metabolome analysis of Arabidopsis plants. Plant Physiol 138(1):304-318

Nordström A, Want E, Northen T, Lehtiö J, Siuzdak G (2008) Multiple ionization mass spectrometry strategy used to reveal the complexity of metabolomics. Anal Chem 80(2):421-429 
Ohta D, Shibata D, Kanaya S (2007) Metabolic profiling using Fourier-transform ioncyclotron-resonance mass spectrometry. Anal Bioanal Chem 389(5):1469-1475

Oikawa A, Nakamura Y, Ogura T, Kimura A, Suzuki H, Sakurai N, Shinbo Y, Shibata D, Kanaya S, Ohta D (2006) Clarification of pathway-specific inhibition by Fourier transform ion cyclotron resonance/mass spectrometry-based metabolic phenotyping studies. Plant Physiol 142(2):398-413

Oliver SG, Winson MK, Kell DB, Baganz F 1998. Systematic functional analysis of the yeast genome. Trends in Biotechnology 16: 373-378

Osbourn AE (1999) Antimicrobial phytoprotectants and fungal pathogens. Fung Gen Biol 26:163-168

Overy SA, Walker HJ, Malone S, Howard TP, Baxter CJ, Sweetlove LJ, Hill SA, Quick WP. (2005). Application of metabolite profiling to the identification of traits in a population of tomato introgression lines. J Exp Bot 56:287-296

Pan X, Welti R, Wang X (2008) Simultaneous quantification of major phytohormones and related compounds in crude plant extracts by liquid chromatography-electrospray tandem mass spectrometry. Phytochemistry 69(8):1773-1781

Pedras MSC and Khan AQ (1997) Unprecedented detoxification of the phytoalexin camalexin by a root rot pathogen. Bioorg Med Chem Lett 7:2255-2260

Pedras MSC, Khan AQ, Taylor JL (1998) Phytoalexin camalexin is not metabolized by $P$. lingam, A. brassicae, or phytopathogenic bacteria. Plant Sci 139:1-8

Pedras MS, Okanga FI, Zaharia IL, Khan AQ (2000) Phytoalexins from crucifers: synthesis, biosynthesis, and biotransformation. Phytochemistry 53(2):161-176 
Pedras MS, Nycholat CM, Montaut S, Xu Y, Khan AQ (2002) Chemical defenses of crucifers: elicitation and metabolism of phytoalexins and indole-3-acetonitrile in brown mustard and turnip. Phytochemistry 59(6):611-625

Pedras MS and Ahiahonu PW (2005) Metabolism and detoxification of phytoalexins and analogs by phytopathogenic fungi. Phytochemistry 66(4):391-411

Pedras MS, Adio AM, Suchy M, Okinyo DP, Zheng QA, Jha M, Sarwar MG (2006) Detection, characterization and identification of crucifer phytoalexins using highperformance liquid chromatography with diode array detection and electrospray ionization mass spectrometry. J Chromatogr A 1133(1-2):172-183

Pedras MS, Zheng QA, Gadagi RS, Rimmer SR (2008) Phytoalexins and polar metabolites from the oilseeds canola and rapeseed: differential metabolic responses to the biotroph Albugo candida and to abiotic stress. Phytochemistry 69(4):894-910

Pegg GF (1965) Phytotoxin production by Verticillium albo-atrum Reinke and Berthold. Nature 208:1228-1229

Pegg GF and Cronshaw DK(1976) Relationship of in vitro to in vivo ethylene production in Pseudomonas solanacearum infection of tomato. Physiol Plant Pathol 9:145-154

Pegg GF, Gull K, Newsam RJ (1976) Transmission electron microscopy of Verticillium albo-atrum hyphae in xylem vessels of tomato plants. Physiol Plant Pathol 8:221-224

Pegg GF and Brady BL (eds.) (2002a) Hosts. In: Verticillium wilts. Wallingford, UK: CABI Publishing 193-340

Pegg GF and Brady BL (eds.) (2002b) Pathogenesis. In: Verticillium wilts. Wallingford, UK: CABI Publishing 142-166

Roepenack-Lahaye E, Degenkolb T, Zerjeski M, Franz M, Roth U, Wessjohann L, Schmidt J, Scheel D, Clemens S (2004) Profiling of Arabidopsis Secondary Metabolites by 
Capillary Liquid Chromatography Coupled to Electrospray Ionization Quadrupole Timeof-Flight Mass Spectrometry. Plant Physiol 134: 548-559

Roessner U, Wagner C, Kopka J, Trethewey RN, Willmitzer L (2000) Technical advance: simultaneous analysis of metabolites in potato tuber by gas chromatography-mass spectrometry. Plant J 23(1):131-142

Roessner U, Luedemann A, Brust D, Fiehn O, Linke T, Willmitzer L, Fernie A (2001) Metabolic profiling allows comprehensive phenotyping of genetically or environmentally modified plant systems. Plant Cell 13(1):11-29

Roessner U, Willmitzer L, Fernie AR (2002) Metabolic profiling and biochemical phenotyping of plant systems. Plant Cell Rep 21(3):189-196

Rygulla W, Seyis F, Lühs W, Eynck C, von Tiedemann A, Friedt W, Snowdon RJ (2007) Combination of resistance to Verticillium longisporum from zero erucic acid Brassica oleracea and oilseed Brassica rapa genotypes in resynthesized rapeseed (Brassica napus) lines. Phytopathology 97 (11):1391-1396

Sanchez DH, Siahpoosh MR, Roessner U, Udvardi M, Kopka J (2008) Plant metabolomics reveals conserved and divergent metabolic responses to salinity. Physiol Plant 132(2):209219

Schauer N, Zamir D, Fernie AR (2005) Metabolic profiling of leaves and fruit of a wild species tomato: a survey of the Solanum lycopersicum complex. J Exp Bot 56:298-307

Schmelz EA, Engelberth J, Alborn HT, O'Donnell P, Sammons M, Toshima H, Tumlinson JH III (2003) Simultaneous analysis of phytohormones, phytotoxins, and volatile organic compounds in plants. Proc Natl Acad Sci U S A 100(18):10552-10557

Schnathorst WC (1981) Life cycle and epidemiology in Verticillium. In: Mace M.C., Bell A.A and Beckman C.H. (eds) Fungal wilt diseases in plants. Academic Press, New York. pp 81-112 
Sellam A, Poupard P, Simoneau P (2006) Molecular cloning of AbGstl encoding a glutathione transferase differentially expressed during exposure of Alternaria brassicicola to isothiocyanates. FEMS Microbiol Lett 258(2):241-249

Sexton AC and Howlett BJ (2000) Characterisation of a cyanide hydratase gene in the phytopathogenic fungus Leptosphaeria maculans. Mol Gen Genet 263(3):463-470

Shulaev V, Silverman P, Raskin I (1997) Airborne signalling by methyl salicylate in plant pathogen resistance. Nature 385:718-721

Shulaev V (2006) Metabolomics technology and bioinformatics. Brief Bioinform 7(2):128139

Smith CA, Want EJ, O'Maille G, Abagyan R, Siuzdak G (2006) XCMS: processing mass spectrometry data for metabolite profiling using nonlinear peak alignment, matching, and identification. Anal Chem 78(3):779-787

Splivallo R, Bossi S, Maffei M, Bonfante P (2007) Discrimination of truffle fruiting body versus mycelial aromas by stir bar sorptive extraction. Phytochemistry 68(20):2584-2598

Staiger D (2002) Chemical strategies for iron acquisition in plants. Angew Chem Int Ed Engl 41(13):2259-2264

Stark C (1961) Das Auftreten der Verticillium - Tracheomykosen in Hamburger Gartenbaukulturen. Gartenbauwissenschaft 26:493-528

Steuer R, Kurths J, Fiehn O, Weckwerth W (2003) Observing and interpreting correlations in metabolomic networks. Bioinformatics 19(8):1019-1026

Steventon LA, Fahleson J, Hu Q, Dixelius C (2002) Identification of the causal agent of Verticillium wilt of winter oilseed rape in Sweden. V. longisporum. Mycological Research 106:570-578 
Tan JW, Bednarek P, Liu JK, Schneider B, Svatos A, Hahlbrock K (2004) Universally occurring phenylpropanoid and species-species indolic metabolites in infected and uninfected Arabidopsis thaliana roots and leaves. Phytochemistry 65:691-699

Thaler JS, Owen B, Higgins VJ (2004) The role of the jasmonate response in plant susceptibility to diverse pathogens with a range of lifestyles. Plant Physiol 135(1):530-538

Thomma BP, Nelissen I, Eggermont K, Broekaert WF (1999) Deficiency in phytoalexin production causes enhanced susceptibility of Arabidopsis thaliana to the fungus Alternaria brassicicola. Plant J 19(2):163-171

Tierens KFM-J, Thomma BPHJ, Brouwer M, Schmidt J, Kistner K, Porzel A, Mauch-Mani B, Cammue BPA, Broekaert WF (2001) Study of the role of antimicrobial glucosinolatederived isothiocyanates in resistance of Arabidopsis to microbial pathogens. Plant Physiol 125:1688-1699

Tikunov Y, Lommen A, de Vos CH, Verhoeven HA, Bino RJ, Hall RD, Bovy AG (2005) A novel approach for nontargeted data analysis for metabolomics. Large-scale profiling of tomato fruit volatiles. Plant Physiol 139(3):1125-1137

Tjamos SE, Flemetakis E, Paplomatas EJ, Katinakis P (2005) Induction of resistance to Verticillium dahliae in Arabidopsis thaliana by the biocontrol agent K-165 and pathogenesis-related proteins gene expression. Mol Plant Microbe Interact 18:555-561

Urbanczyk-Wochniak E and Fernie AR (2005) Metabolic profiling reveals altered nitrogen nutrient regimes have diverse effects on the metabolism of hydroponically-grown tomato (Solanum lycopersicum) plants. J Exp Bot 56(410):309-321

Van Etten HD, Matthews DE, Matthews PS (1989) Phytoalexin detoxification: importance for pathogenicity and practical implications. Annu Rev Phytopathol 27:143-164 
Veronese P, Narasimhan ML, Stevenson RA, Zhu JK, Weller SC, Subbarao KV, Bressan RA (2003) Identification of a locus controlling Verticillium disease symptom response in Arabidopsis thaliana. Plant J 35:574-587

Wagner S, Scholz K, Sieber M, Kellert M, Voelkel W (2007) Tools in metabonomics: an integrated validation approach for LC-MS metabolic profiling of mercapturic acids in human urine. Anal Chem 79(7):2918-2926

Walling L (2000) The myriad plant responses to herbivores. J Plant Growth Regul 19:195216

Wang J-Y, Cai Y, Gou J-Y, Mao Y-B, Xu Y-H, Jiang W-H, Chen X-Y (2004) VdNEP, an elicitor from Verticillium dahliae, induces cotton plant wilting. Appl Environ Microbiol 70:4989-4995

Wilhelm S (1955) Longevity of the Verticillium wilt fungus in the laboratory and field. Phytopathology 45:180-181

Williams JS, Hall SA, Hawkesford MJ, Beale MH, Cooper RM (2002) Elemental sulfur and thiol accumulation in tomato and defense against a fungal vascular pathogen. Plant Physiol 128(1):150-159

Windig W, Phalp JM, Payne AW (1996) A noise and background reduction method for component detection in liquid chromatography mass spectrometry. Anal Chem 68(20):3602-3606

Windig W and Smith WF (2007) Chemometric analysis of complex hyphenated data. Improvements of the component detection algorithm. J Chromatogr A 1158(1-2):251-257

Wolfender JL, Ndjoko K, Hostettmann K (2003) Liquid chromatography with ultraviolet absorbance-mass spectrometric detection and with nuclear magnetic resonance spectroscopy: a powerful combination for the on-line structural investigation of plant metabolites. J Chromatogr A 1000(1-2):437-455 
Zhou L, Hu Q, Johansson A, Dixelius C (2006) Verticillium longisporum and Verticillium dahliae: infection and disease in Brassica napus. Plant Pathol 55:137-144

Zeise K and von Tiedemann A (2001) Morphological and physiological differentiation among vegetative compatibility groups of Verticillium dahliae in relation to Verticillium longisporum. J Phytopathol 149:469-475

Zeise K and von Tiedemann A (2002) Host specialization among vegetative compatibility groups of Verticillium dahliae in relation to V. longisporum. J Phytopathol 150:112-119 


\section{Danksagung}

An erster Stelle möchte ich mich ganz besonders bei meinem Doktorvater Herrn Prof. Dr. Petr Karlovsky für die Überlassung des Themas, die Unterstützung und Freiheit bei der Durchführung dieser Arbeit sowie für die Durchsicht der Manuskripte bedanken. Die stete Hilfs- und Diskussionsbereitschaft sind besonders zu erwähnen.

Bei Herrn Prof. Dr. Andreas von Tiedemann bedanke ich mich für die bereitwillige Übernahme des Korreferates.

Frau Dr. Ursula Hettwer möchte ich ganz herzlich für die äußerst angenehme Zusammenarbeit und Verständigung im Labor danken, die auch in Abwesenheit des anderen funktionierte, da man sich intuitiv aufeinander verlassen konnte. Ihre immerwährende positive und optimistische Einstellung hat mich zwischendurch immer wieder aufgemuntert.

Meinen Projektpartnern Dr. Nadine Riediger und Dr. Hernán Laurentin danke ich für die jeweils fruchtbare Zusammenarbeit und Diskussionsbereitschaft. Herrn Dr. Reza Khorassani danke ich für die zur Verfügung gestellten Proben.

Der Deutschen Forschungsgemeinschaft (DFG) danke ich für die Finanzierung dieser Arbeit im Rahmen der Forschergruppe FOR 546 „Verticillium“, die zum regelmäßigen regen Austausch beitrug.

Mein Dank gilt auch der Varian Deutschland GmbH, namentlich Frau Dr. Susanne Sölter und Herrn Dr. Oliver Landgraf, für die exzellente technische Unterstützung, Beratung und gute Zusammenarbeit in Bezug auf die 500-MS Ion Trap und TurboDDS ${ }^{\mathrm{TM}}$.

Besonders bedanken möchte ich mich bei der AG Prof. Dr. Ivo Feußner für die Bereitstellung der Ionenfalle im ersten Jahr, die mir die Grundlage zur Etablierung der Datenauswertung sicherte.

Bei Heike Rollwage, Sabine Nutz, Stefanie Griebel und Dr. Cornelia Göbel möchte ich mich ganz herzlich für das kurzfristige Korrekturlesen und die Unterstützung bei der Formatierung der Dissertation bedanken.

Dem gesamten Institut für Pflanzenpathologie und Pflanzenschutz danke ich für die freundliche Aufnahme und das tolle Arbeitsklima.

Heike Rollwage, Patricia Bartoschek, Monika Bossmann, Claudia Nordmann, Ines Kunze, Ulrike Vetterling, Nadine Reimann, Solange Kugler bedanke ich mich ganz herzlich für die technische Unterstützung, vor allem bei der langwierigen Gewinnung des Xylemsaftes.

Bei meiner Familie, insbesondere meiner Mutter, und meinen Freunden möchte ich mich für das große Verständnis, die Geduld und moralische Unterstützung ganz herzlich bedanken. 


\section{Publications from this work}

\section{Refereed Journals}

Laurentin H, Ratzinger A, Karlovsky P (2008) Relationship between metabolic and genomic diversity in sesame (Sesamum indicum L.). BMC Genomics, accepted

Sirrenberg A, Göbel C, Grond S, Czempinski N, Ratzinger A, Karlovsky P, Santos P, Feussner I, Pawlowski K (2007) Piriformospora indica affects plant growth by auxin production. Physiol Plant. 131(4):581-589

\section{Manuscripts from this work}

Khorassani R, Hettwer U, Ratzinger A, Steingrobe B, Karlovsky P, Claassen N (2008) Identification of soil $\mathrm{P}$ mobilizing components of sugar beet root exudates after differential metabolic profiling by HPLC-MS. submitted

Tappe H, Floerl S, Thurow C, Carsjens JG, Ratzinger A, Dilcher M, Karlovsky P, Feussner I, Polle A, Gatz C (2008) Expression Profiling of Arabidopsis thaliana Plants after infection with the xylem-colonizing Fungus Verticillium longisporum. submitted

Ratzinger A, Riediger N, von Tiedemann A, Karlovsky P (2008) Identification of salicylic acid glucoside in xylem sap of Brassica napus and the effect of infection with Verticillium longisporum. submitted

Ratzinger A, Riediger N, von Tiedemann A, Karlovsky P (2008) Differential metabolic profiling of xylem sap of Brassica napus infected with Verticillium longisporum. in preparation

\section{Conference Proceedings}

Samal D, Ratzinger A, Karlovsky P, Steingrobe B, Sadana US, Claassen N (2006) Potassium efficiency mechanisms in relation to exudation rate and composition of root exudates of wheat and sugar beet grown at low and high K levels. Proceedings of the International Symposium on Balanced Fertilization for Sustaining Crop Productivity. Punjab Agricultural University, Ludhiana, India, 22.-25.11.2006.

\section{Presentations at national and international conferences}

Ratzinger A, Riediger N, von Tiedemann A, Karlovsky P (2007) Metabolic signals occurring in Brassica napus xylem sap after infection with Verticillium longisporum. Oral presentation on Meeting on Signal exchange between root and microorganisms. Göttingen, Germany, 07.-09.03.2007, Book of Abstracts. 
Ratzinger A, Weiberg A, Karlovsky P (2007) Transformation of secondary metabolites of Brassica napus by vascular pathogen Verticillium longisporum. 8th VAAM (Association for General and Applied Microbiology) Conference "Molecular Biology of Fungi", Hamburg, Germany, 23.-25.09.2007, Conference Book.

Lendzion J, Ratzinger A, Karlovsky P, Leuschner C (2007) The effect of low atmospheric moisture on abscisic acid accumulation in woodland herbs. 125 years German Botanical Society, Botanikertagung, Hamburg, Germany, 03.-07.09.2007, Book of Abstracts.

Ratzinger A, Riediger N, von Tiedemann A, Karlovsky P (2007) Identification of metabolites in Brassica napus xylem sap affected by Verticillium longisporum infection using HPLC-DAD-ESI-MS. Poster presentation on $40^{\text {th }}$ Annual Meeting of the German Society for Mass Spectrometry, Bremen, Germany, 11.-14.03.2007, Book of Abstracts.

Carsjens JG, Tappe H, Flörl S, Ratzinger A, Karlovsky P, Polle A, Gatz C, Feussner I (2007) Phytohormone responses in the Verticillium longisporum - Arabidopsis thaliana pathosystem. Poster presentation on Meeting on Signal exchange between root and microorganisms, Göttingen, Germany, 07.-09.03.2007, Book of Abstracts.

Ratzinger A, Karlovsky P (2006) Sekundärmetaboliten von Verticillium longisporum in der Interaktion mit Brassica napus; Secondary metabolites of Verticillium longisporum. Poster presentation on $55^{\text {th }}$ German Congress of Plant Protection. Göttingen, Germany, 25.-28.09.2006, Mitt. Biol. Bundesanst. Land- Forstwirtsch. 400:262.

Hiegl W, Wolf HC, Ratzinger A, Karlovsky P (2006) Functional analysis of a new polyketide synthase gene in Verticillium longisporum. Poster presentation on $55^{\text {th }}$ German Congress of Plant Protection. Göttingen, Germany, 25.-28.09.2006, Mitt. Biol. Bundesanst. Land- Forstwirtsch. 400:217.

Riediger N, Koopmann B, Karlovsky P, Ratzinger A, Marsh D, von Tiedemann A (2006) Zur Rolle von Stickoxid (NO) als Signal in der Interaktion von Brassica napus mit Verticillium longisporum; Towards the role of nitric oxide (NO) as a signal in the interaction of Brassica napus with Verticillium longisporum. Poster presentation on $55^{\text {th }}$ German Congress of Plant Protection. Göttingen, Germany, 25.-28.09.2006, Mitt. Biol. Bundesanst. Land- Forstwirtsch. 400:252.

Wolf HC, Hiegl W, Ratzinger A, Karlovsky P (2006) Functional and phylogenetic analysis of a new polyketide synthase gene in Verticillium longisporum. Poster presentation on Annual Meeting of the Association for General and Applied Microbiology (VAAM), Jena, Germany, 19.-22.03.2006, Biospektrum 2006 Sonderausgabe.

Ratzinger A, Riediger N, von Tiedemann A, Karlovsky P (2006) Differential profiling of metabolites synthesized in the interaction between Verticillium longisporum and Brassica napus by HPLC-MS. Poster presentation on $39^{\text {th }}$ Annual Meeting of the German Society for Mass Spectrometry, Mainz, Germany, 05.-08.03.2006, Book of Abstracts.

Laurentin H, Ratzinger A, Karlovsky P (2005) Relationship between metabolic and genotype diversity in sesame (Sesamum indicum L.). Poster presentation on 2nd 
International Symposium on Recent Advances in Food Analysis, Prague, Czech Republic, 02.-04.11.2005, Book of Abstracts.

Ratzinger A, Riediger N, von Tiedemann A, Karlovsky P (2005) Secondary metabolites in interactions between Verticillium longisporum and its plant hosts. Poster presentation on Annual Meeting of the German Genetics Society, Braunschweig, Germany, 21-24.09.2005, Genes, Chromosomes, Genomes (2005) 10:49. 


\section{Curriculum Vitae}

\section{Personal Data}

$\begin{array}{ll}\text { Name: } & \text { Astrid Ratzinger } \\ \text { Date of birth: } & 08.02 .1980 \\ \text { Place of birth: } & \text { Berlin, Germany } \\ \text { Family status: } & \text { unmarried } \\ \text { Place of residence: } & \text { Göttingen, Germany } \\ \text { E-mail: } & \text { aratzin at gwdg dot de }\end{array}$

\section{Secondary education}

09/1986-06/1999 Primary school and grammar school (Pasteur-Oberschule), Berlin, Germany; Graduation: High school diploma

\section{Tertiary education}

03/2005 - 05/2008 Doctoral research at the Department for Crop Sciences, Molecular Phytopathology and Mycotoxin Research Unit, Georg-AugustUniversity, Göttingen, Germany, under the guidance of Prof. Dr. Petr Karlovsky

11/2004 Diploma in chemistry

01/2004 - 10/2004 Diploma thesis at the Charité Berlin, Institute for Legal Medicine, Humboldt-Universität zu Berlin, Berlin, Germany, under the guidance of Prof. Dr. F. Pragst / Prof. Dr. M. Linscheid 10/1999 - 12/2003 Studies of chemistry at Humboldt-Universität zu Berlin, Berlin, Germany; Specialization in analytical chemistry

\section{Professional work}

03/2003 - 02/2005 Tutor for undergraduates at Analytical and Environmental Chemistry group (Prof. Dr. M. Linscheid), Department of Chemistry, Humboldt-Universität zu Berlin, Berlin, Germany 


\section{Eidesstattliche Erklärung}

Hiermit erkläre ich eidesstattlich, dass diese Dissertation selbständig und ohne unerlaubte Hilfe angefertigt wurde.

Göttingen, im Mai 2008 